Burkina Faso: Second Review Under the Extended Credit Facility Arrangement and Request for Waiver of Nonobservance of a Performance Criterion-Press Release; Staff Report; and Statement by the Executive Director for Burkina Faso 


\section{INTERNATIONAL MONETARY FUND}

July 2019

\section{BURKINA FASO}

IMF Country Report No. 19/257

\section{SECOND REVIEW UNDER THE EXTENDED CREDIT FACILITY ARRANGEMENT AND REQUEST FOR WAIVER OF NONOBSERVANCE OF A PERFORMANCE CRITERION- PRESS RELEASE; STAFF REPORT; AND STATEMENT BY THE EXECUTIVE DIRECTOR FOR BURIKINA FASO}

In the context of the Second Review Under the Extended Credit Facility Arrangement and Request for Waiver of Nonobservance of a Performance Criterion, the following documents have been released and are included in this package:

- A Press Release including a statement by the Chair of the Executive Board.

- The Staff Report prepared by a staff team of the IMF for the Executive Board's consideration on July 19, 2019, following discussions that ended on May 29, 2019, with the officials of Burkina Faso on economic developments and policies underpinning the IMF arrangement under the Extended Credit Facility. Based on information available at the time of these discussions, the staff report was completed on June 27, 2019.

- A Supplementary Information and Supplementary Letter of Intent.

- A Statement by the Executive Director for Burkina Faso.

The documents listed below have been or will be separately released.

Letter of Intent sent to the IMF by the authorities of Burkina Faso*

Memorandum of Economic and Financial Policies by the authorities of Burkina Faso*

Technical Memorandum of Understanding*

*Also included in Staff Report

The IMF's transparency policy allows for the deletion of market-sensitive information and premature disclosure of the authorities' policy intentions in published staff reports and other documents.

Copies of this report are available to the public from

International Monetary Fund • Publication Services

PO Box 92780 - Washington, D.C. 20090

Telephone: (202) 623-7430 • Fax: (202) 623-7201

E-mail: publications@imf.org Web: http://www.imf.org

Price: $\$ 18.00$ per printed copy

\section{International Monetary Fund Washington, D.C.}


Press Release No. 19/292

FOR IMMEDIATE RELEASE

July 19, 2019
International Monetary Fund

$70019^{\text {th }}$ Street, NW

Washington, D. C. 20431 USA

\section{IMF Executive Board Completes the Second Review under the Extended Credit Facility Arrangement and Approves US\$25 Million Disbursement to Burkina Faso}

On July 19, 2019, the Executive Board of the International Monetary Fund (IMF) completed the second review of Burkina Faso's economic performance under a three-year program supported by the IMF's Extended Credit Facility (ECF). Completion of the review enables the disbursement of the equivalent of SDR18.06 million (about US\$25 million), bringing total disbursements under the arrangement to the equivalent of SDR54.18 million (about US\$75 million). The Board also approved the authorities' request for waivers for nonobservance of two performance criteria.

Burkina Faso's three-year ECF-supported program for the equivalent of SDR108.36 million (about US\$149.9 million or 90 percent of the country's quota at the time of approval of the arrangement), was approved on March 14, 2018 (see Press Release No. 18/86). A key objective of the program is to create fiscal space for priority spending by strengthening revenue mobilization, containing current spending and improving the efficiency of public investment.

Following the Executive Board discussion, Mr. Mitsuhiro Furusawa, Deputy Managing Director and Acting Chair, made the following statement:

"Performance under the ECF-supported program has been broadly satisfactory. Economic activity remains resilient in the face of increased security and social tension pressures. Burkina Faso continues to pursue its development goals, and further support from the international community to address security and development needs is important. Additional effort is required to create fiscal space to support further progress in priority areas of development.

"Burkina Faso is expected to meet the WAEMU convergence criteria of fiscal deficit of 3 percent of GDP in 2019. Policy efforts underpinning this objective should focus on broadening the tax base - including by reducing exemptions - improving tax administration, and enhancing the efficiency of expenditure, including by containing the wage bill and subsidies. Public financial management reforms are advancing, and further progress could be achieved by strengthening the coordination between departments of the Ministry of Finance and other institutions.

Washington, D.C. 20431 • Telephone 202-623-7100 • Fax 202-623-6772 • www.imf.org 
"In light of the political uncertainty surrounding the adoption of the authorities' public sector pay reform package, they are now focusing on a two-pronged approach to contain the wage bill. First, in the near term, the authorities will implement transitional measures to keep the wage bill in line with understandings reached at the last review. In this respect, it is of utmost importance that the authorities eschew any new pay awards until a broad reform package is in place, and that they build consensus around the appropriate level of public pay, through the articulation of a public pay strategy to the wider public. Second, in the meanwhile, the authorities will pursue politically feasible ways to adopt their reform package.

"The authorities are taking steps to strengthen the implementation of the automatic fuel price adjustment mechanism while developing social safety nets for the most vulnerable. In this respect, greater transparency, improved communication and the avoidance of discretionary implementation of the mechanism are crucial." 


\section{INTERNATIONAL MONETARY FUND}

\section{BURKINA FASO}

June 27, 2019

\section{SECOND REVIEW UNDER THE EXTENDED CREDIT FACILITY ARRANGEMENT AND REQUEST FOR WAIVER OF NONOBSERVANCE OF A PERFORMANCE CRITERION}

\section{EXECUTIVE SUMMARY}

Context. In light of widespread concern about the security crisis and protracted public sector pay disputes, the government resigned in January 2019. The new government, installed with a mission to combat the security crisis with more vigor, remains under tremendous pressure.

Outlook and Risks. Growth in 2018 remained resilient as a bumper harvest more than compensated for a decline in non-agricultural GDP growth. Inflation remained subdued, and the overall fiscal deficit declined below 5 percent of GDP, though essentially at the cost of lower public investment. Given difficulties in moving ahead with a constitutional reform, the authorities are now focusing on a two-stage strategy to contain the wage bill. First, in the near term, transitional measures will be implemented and the outlook for the wage bill would remain in line with the understandings reached at the first review. Second, in the meanwhile, the authorities will continue exploring politically feasible ways to adopt their reform package. The main risks to the program stem from heightened security risks and further labor disputes.

Program Performance and Policies. Performance under the ECF-supported program approved in March 2018 has been broadly satisfactory. All but one performance criteria were met. The ceiling on net domestic financing was not observed, due to a substantial cash adjustment relative to 2017 operations settled in 2018. The authorities are requesting a waiver for this nonobservance based on corrective measures to enhance the monitoring of investment programming, and improve the intra-year planning and spreading of commitments. All other quantitative targets to end-December 2018 were met, and 8 out of 13 structural benchmarks through mid-June 2019 were met.

Staff's Views. Based on the proposed corrective measures, staff supports the authorities request for a waiver and the release of the third disbursement under the ECF arrangement in the amount of SDR 18.06 million. 
Approved By

Dominique Desruelle (AFR) and Edward R. Gemayel (SPR)
Discussions were held in Ouagadougou during May 16-29, 2019. The staff team comprised Mr. Ahokpossi (head), Mr. Simione, and Ms. Nikaein Towfighian (all AFR), Mr. Gbohoui (FAD), and Mr. KassHanna (SPR). Mr. Jenkinson (Resident Representative) and Mr. Ouattara (local economist) assisted the mission. Mr. Desruelle (AFR) joined the last days of the mission. Mr. Bah (OED) also participated. Administrative support was provided by Ms. Margevich, Ms. Tolbert and Ms. Ouedraogo (all AFR). The team met with Mr. Roch Marc Christian Kaboré, President of Faso; Mr. Christophe Dabiré, Prime Minister; Mr. Lassané Kaboré, Minister of Economy, Finance and Development; Ms. Edith Clémence Yaka, Minister Delagate responsible for the budget; Mr. Stanislas Ouaro, Minister of Education; Mr. Bassirou Kamadji Ly, President of the Finance and Budget Commission of the National Assembly, and his colleagues; and other senior government officials. The mission also met with Mr. Charles Luanga Ki-Zerbo, National Director of the Central Bank of West African States, and development partners.

\section{CONTENTS}

BACKGROUND AND RECENT DEVELOPMENTS

A. Background

B. Recent Developments

PERFORMANCE UNDER THE ECF ARRANGEMENT

POLICY DISCUSSIONS

A. Budget Implementation

B. Enhancing Revenue Mobilization

C. Managing Energy Subsidies

D. Containing the Medium-Term Growth in the Wage Bill

E. Enhancing the Efficiency of Public Spending ___ $\underline{13}$

F. Social Sector Spending ___ 14

G. Governance 


\section{FIGURES}

1. Recent Economic Developments

2. Real and External Developments

3. Fiscal Developments

\section{TABLES}

1. Selected Economic and Financial Indicators, 2016-24 $\underline{22}$

2. Balance of Payments, 2016-24__ 23

3. Monetary Survey, 2016-20__ 24

4. Selected Financial Soundness Indicators, 2012-June 2018__ 25

5a. Consolidated Operations of the Central Government, 2017-24 (CFAF billions)___ _ 26

5b. Consolidated Operations of the Central Government, 2017-24 (Percent of GDP) __ _ 27

5c. Consolidated Operations of the Central Government, 2019 (CFAF billions)___ 28

5d. Consolidated Operations of the Central Government, 2020 (CFAF billions) __ 29

6. External Financing Requirements, 2017-21__ $\underline{30}$

7. Schedule of Disbursements Under Three-year ECF Arrangement, 2018-20___ $\underline{31}$

8. Indicators of Capacity to Repay the IMF, 2017-32__

\section{ANNEX}

I. Risk Assessment Matrix

\section{APPENDIX}

I. Letter of Intent $\underline{34}$

Attachment 1. Memorandum of Economic and Financial Policies $\underline{37}$

Attachment 2. Technical Memorandum of Understanding $\underline{65}$ 


\section{BACKGROUND AND RECENT DEVELOPMENTS}

\section{A. Background}

\section{Security threats have intensified since the final quarter of 2018, resulting in a sharp} rise in displaced people and popular unease (Text Figure 1). The state of emergency remains in place in 7 out of 13 regions. Security fears are heightening ethnic tensions and inter-communal violence. The number of internally displaced people increased from about 40,000 at the end of December 2018 to 170,000 in May 2019 and is expected to exceed 200,000 by year end. At the same time, security disruptions have resulted in the closure of 18 health centers and over 1000 schools, with almost 146,000 pupils affected. Key external stakeholders have expressed heightened concerns about the security situation while an Emergency Program launched by the authorities and the United Nations (UN) is not sufficiently resourced to cover all projected needs.

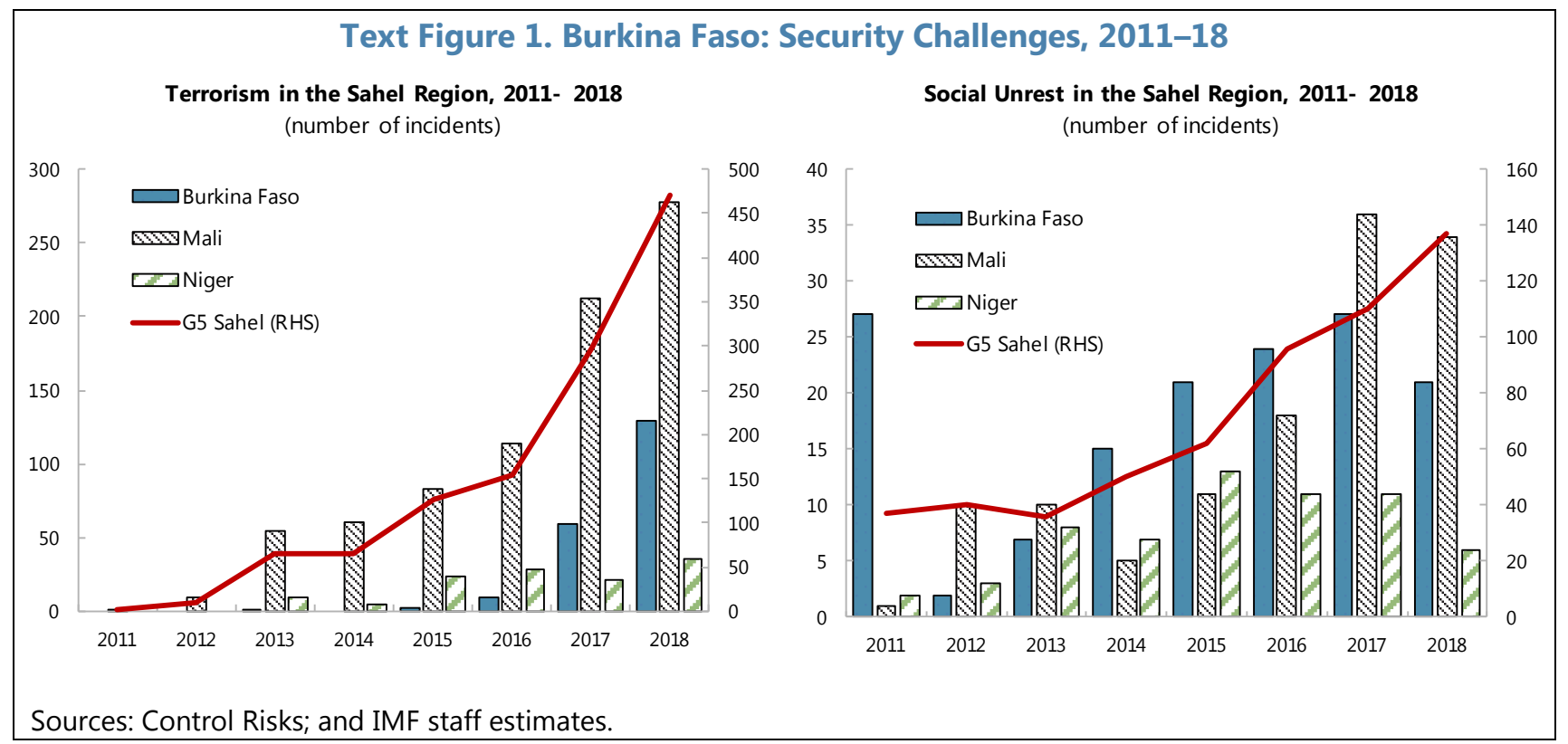

2. Additional external support appears necessary to help the authorities regain control of the security situation and prevent its spread to neighboring countries. Heightened security needs have increased spending pressures (additional 0.6 percent of GDP in 2018 and projected 0.6 percent of GDP in 2019). The security shock is likely to persist, requiring a permanent increase in security capability and additional social spending related to the surging number of displaced people. As the government recovers control of some regions, investment needed to regain the trust of the population, including rebuilding schools, have become urgent. Some neighboring countries that are thus far untouched by terrorist incidents are reporting credible intelligence of spillover risk from Burkina Faso. Amid these challenges, the authorities have intensified international appeals towards securing further financial and logistical support from the UN, and multilateral and bilateral development partners. 
3. Amid the widespread popular dissatisfaction with the security crisis and protracted public sector pay disputes, a new government was installed in January 2019. An initial truce on union action quickly broke down as teachers and then police and health workers entered disputes. Staff in the Ministry of Finance also took action in protest at the reduction in allowances in the 2019 budget. This was resolved in April 2019 with the introduction of a transitional system of bonuses, which would reduce these bonuses by around 60 percent relative to their size in 2018.

\section{B. Recent Developments}

\section{Economic performance remained resilient during 2018, as a bumper harvest} outweighed the impact of labor unrest. Real GDP growth is estimated to have accelerated to 6.8 percent in 2018 (from 6.3 percent in 2017), reflecting essentially a stronger-than-expected rebound in the primary sector (9.9 percent), thanks to strong growth in agriculture (17.2 percent), after a significant contraction in 2017 (-9.7 percent) due to rainfall shocks (Figure 1, Table 1). The strong performance in agriculture was driven by better food harvests (including a 27.5 percent increase in cereals production). This contrasted with a disappointing 29 percent decline in cotton production due to cotton pests and reduced farmers' engagement in the cotton production scheme. Non-agriculture GDP growth slowed to 5.7 percent (from 8.4 percent in 2017) partly due to a substantial contraction in cotton ginning and telecommunications, and growth slowdown in construction, public works and government services amid fiscal consolidation.

\section{Inflation remained subdued at $\mathbf{0 . 3}$ percent year-on-year $(y / y)$ in December 2018} ( 2 percent on average) and became negative in early 2019. Better food harvests helped push food prices down in the last quarter of 2018 and early 2019. Despite the upward adjustment in fuel prices in November 2018, the authorities' agreement with transport operators, under which operating margins were reduced, has so far helped to prevent the pass-through of higher fuel prices to transport fares. Annual y/y and average inflation in April 2019 stood at -2.5 percent and 0.6 percent, respectively, although monthly food inflation ( 0.7 percent) has started to catch up since March 2019.

\section{The overall fiscal deficit declined from 7.8 percent of GDP in 2017 to 4.9 percent of} GDP in 2018, albeit essentially at the cost of lower investment. Total revenue, excluding grants, slightly improved to 19.5 percent of GDP in 2018 from 19.4 percent in 2017, surpassing the revised program target of 18.8 percent as revenue collection recovered strongly in the final months of the year to make-up for shortfalls experienced through earlier strikes at the Ministry of Finance (MoF). Given the rise in the wage bill from 8.6 percent in 2017 to 9.0 percent in 2018, the consolidation was achieved mainly through a sharp reduction in investment (2 percent of GDP lower than in 2017) and current transfers (1.5 percent of GDP lower than in 2017).

7. Private sector credit growth slowed down in 2018. Growth of total credit to the economy accelerated to 12.4 percent (from 10.5 percent in 2017), mainly reflecting a strong growth (181 percent) of credit to public corporations, in part due to a 60 billion CFAF securitization of state debt to the state oil importing company, SONABHY. As for the private sector, credit growth declined to 6.7 percent in 2018 from 14.4 percent in 2017 and remained subdued at 5.4 percent at end- 
March 2019. The banking system remained broadly stable with the aggregate capital level above prudential requirements and non-performing loans contained at their end-2017 level (8.7 percent).

\section{The current account deficit narrowed to 5.8 percent of GDP in 2018, down from} 7.3 percent of GDP in 2017, contributing to strengthen regional reserves. The deficit is mostly driven by a persistent deficit in the balance of services, which improved in 2018 to 6.7 percent of GDP, down from 7.2 percent in 2017. The trade balance improved to a surplus of 0.7 percent of GDP, as an increase in imports of primary goods, especially oil (24.5 percent), was largely offset by an increase in the exports of gold (8.5 percent) and of agricultural products, except cotton (28.2 percent). Moreover, the government was able in 2018 to attract higher official transfers (10.2 percent), particularly in the form of budget support grants. These developments, along with Eurobond issuances in some WAEMU countries, contributed to boost the level of regional reserves, which stood at 4.4 months of imports in 2018.

\section{PERFORMANCE UNDER THE ECF ARRANGEMENT}

\section{Performance under the ECF-supported program has been broadly satisfactory. All but} one performance criteria, as well as all indicative targets at end-December 2018 were met (MEFP Table 1). Progress on structural reforms has also been satisfactory, with 8 out of 13 benchmarks through mid-June 2019 having been met (MEPF Table 2).

\section{The ceiling for net domestic financing at end-December 2018 was breached by CFAF} 15 billion despite meeting the indicative target for the overall deficit. The end-March 2019 indicative target for net domestic financing was not met either. This reflects the impact of the substantial cash adjustment to the overall deficit on a commitment basis, which mainly resulted from paying down in 2018 a large stock of short-term outstanding commitments from 2017 (the stock declined from CFAF 110.8 billion at end-December 2017 to CFAF 20.6 billion at end-December 2018). ${ }^{1}$ The government is seeking a waiver for the non-observance of this performance criterion based on its corrective measures to enhance the monitoring of investment programming, and improve the intra-year planning and spreading of commitments (see LOI and MEFP).

\section{The implementation of structural measures has been satisfactory (Table 8). The} significant delay in establishing the Treasury Single Account (TSA) relates to weaknesses in underlying accounting systems and procuring the necessary software interface with the central bank. As for online asset declarations of government officials, a tender has been launched for the development of the IT platform and acquisition of storage servers. In terms of rebasing the national accounts, the rebased nominal estimates have been published for year 2015 (the new base year) and work is underway to finalize and publish the series from 1999 to 2014 and from 2016 onward. As part of measures to help contain the wage bill, the publication of the number of civil servants by,

\footnotetext{
${ }^{1}$ These short-term non-liquidated commitments (DENOs or dépenses engagées non ordonnancées in French) relate to expenditure commitments that have not yet been validated for payment. 
grades, regions and ministry, a structural benchmark for April 2019, is now a prior action. Other structural benchmarks have been met.

\section{ECONOMIC OUTLOOK AND RISKS}

\section{Growth is projected to moderate to $\mathbf{6}$ percent in $\mathbf{2 0 1 9}$ and in the medium term.}

Agriculture sector growth is expected to revert towards normal levels reflecting the 2018 base year effect, but in 2019 will remain above the long-term average owing to the envisaged big rebound in cotton production. While mining sector output growth has declined in the first quarter of 2019, the sector should remain an important source of growth in the medium term with the coming on stream of one new mine in 2019 and two in 2020. Growth in 2019 in the non-agriculture economy is projected to remain at about the same level as in 2018 as the growth of credit to the private sector is expected to stabilize.

13. The inflation outlook is benign. Average inflation is projected to remain within the WAEMU convergence criterion of 3 percent. Better food harvests in 2018, which contributed to food price deflation in late 2018 and early 2019, are expected to keep inflationary pressures contained. The positive agriculture sector outlook for 2019 is an additional potential source of low inflation.

14. The overall fiscal deficit is expected to narrow to 3 percent of GDP, consistent with the WAEMU convergence criterion. The overall budget for 2019 is predicated on efforts to improve tax revenue mobilization to around 17.8 percent of GDP in 2019, slightly higher than the realization from 17.3 percent in 2018. The wage bill is projected to rise to 9.9 percent in GDP (from 9 percent in 2018) reflecting the materialization of a set of agreements reached by the government and different labor unions in 2018 and earlier.

15. In 2019, the current account is expected to widen moderately, but should resume its narrowing trend over the medium-run. Staff projects the current account deficit to widen to 6.1 percent of GDP in 2019, driven by a projected decrease of cotton exports (reflecting the sharp drop in production of cotton in 2018 combined with projected lower cotton prices in 2019), and lower production of gold in the first quarter of the year. Over the medium term, the overall narrowing trend of the current account deficit is expected to continue, reflecting the effects of fiscal consolidation on import demand and a resumption of steady growth in the extractive industries and agricultural sectors.

16. The balance of risks is tilted to the downside. The nature of risks remains unchanged, but downside risks to growth projections have increased relative to the first review. The main domestic risk remains of further terrorist activity and further labor disputes (Annex 1). Security threats could weigh on mining, tourism, and government revenue and expenditure. The latter would add to pressures for increased current spending. On the external side, Burkina Faso would be adversely affected by the spillovers from intensified protectionism and downside risks to global growth, which could negatively affect exports and external aid. 


\section{POLICY DISCUSSIONS}

\section{A. Budget Implementation}

17. Tax revenue projections are in line with the outlook at the time of the first review, but risks of revenue shortfall remain. Industrial actions by staff of the ministry of finance affected revenue performance in the first quarter. Even though the program revenue target was met, the authorities' internal markers indicate that revenue collection was below potential, and a continuation of industrial actions would jeopardize performance for the year. Revenue in the first quarter was supported by higher income tax from the mining sector, reflecting a strong corporate performance in 2018, and online filing and telepayments by big enterprises. Going forward, revenue collection from small and medium enterprises requires more tax administration efforts. Industrial actions by staff of the ministry of finance have stopped following an agreement between the latter and the government that contains the "Prime de Motivation" bonuses (previously known as "Fonds Communs") to about 23 billion CFAF (or about 40 percent of their size in 2018, 55 billion CFAF). With this agreement in place, the authorities are confident that tax revenue objectives for 2019 will be met. The implementation of tax measures included in the 2019 budget, which was timid thus far, will accelerate.

\section{A revenue windfall from telecommunication licenses in the first half of $\mathbf{2 0 1 9}$ is} expected to boost overall revenue and create room for additional investment and security spending, while making it easier to meet the WAEMU fiscal deficit ceiling of $\mathbf{3}$ percent of GDP (Text Table 1). Windfall telecommunication license payments of 40 billion CFAF helped boost total revenue in the first quarter to 396.4 billion CFAF (18.7 percent higher year-on-year basis). Another payment of 80 billion CFAF was received in the second quarter, raising the total windfall to 1.4 percent of GDP. The authorities indicated that some of the windfall will finance higher security spending ( 0.6 percent of GDP) and agreed with staff that the remaining would be allocated to the much-needed investment. As a result, staff estimates that investment could be around 5 percent of GDP, compared to 3.6 percent of GDP in the 2019 budget. The total windfall is untouched thus far, and its allocation will be decided at the time of the adoption of the updated budget for 2019 (Loi de finance rectificative) by September 2019. In 2020, absent a similar windfall, total revenue and grants would decline to 23.1 percent of GDP (from 24.4 percent of GDP in 2019), although tax revenue should continue to grow by 0.5 percent of GDP, in part reflecting the full-year effect of the revenue measures implemented during 2019. ${ }^{2}$

\section{Other expenditures are expected to remain broadly in line with the adopted 2019} budget (Text Table 1). Current expenditure is projected to increase relative to 2018, due to higher wages and salaries ( 0.9 percent of GDP) and transfers ( 0.6 percent of GDP) due to a 66 billion CFAF provision to clear subsidy arrears to SONABHY accumulated in the second half of 2018. On the other hand, goods and services would be lower by 0.2 percent of GDP. Wages and salaries are expected to

\footnotetext{
2 These include additional taxes on non-alcoholic beverages, airline tickets, improvement in customs procedures and the opening of new tax offices (IMF Report No 19/15, EBS/18/125).
} 
start declining in 2020, while the implementation of the fuel price mechanism would limit subsidies to cover the anticipated losses to the state-owned fuel importing company (SONABHY).

\section{Sustaining macroeconomic stability through fiscal consolidation remains an} immediate priority for Burkina Faso. Public debt increased from 38.4 percent of GDP in 2017 to 42.9 percent of GDP in 2018 but remains manageable. Given the need to scale security spending to address the gauntlet of terrorism, preserve priority investment, and protect the most vulnerable, the authorities should opt for a multipronged approach: (i) enhance revenue mobilization; (ii) contain recurrent spending, particularly the wage bill; (iii) improve spending efficiency; and (iv) eliminate energy subsidies and channel resources toward more important social spending.

\begin{tabular}{|c|c|c|c|c|}
\hline \multicolumn{5}{|c|}{$\begin{array}{l}\text { Text Table 1. Burkina Faso: Consolidated Op } \\
\qquad \begin{array}{r}\text { Government, 2017-20 } \\
\text { (percent of GDP) }\end{array}\end{array}$} \\
\hline & 2017 & 2018 & 2019 & 2020 \\
\hline & Out. & Prel. & Proj. & Proj \\
\hline Total revenue and grants & 22.1 & 22.2 & 24.4 & 23.1 \\
\hline Total revenue & 19.4 & 19.5 & 21.3 & 20.4 \\
\hline Tax & 17.3 & 17.3 & 17.8 & 18.3 \\
\hline Nontax & 2.1 & 2.2 & 3.5 & 2.1 \\
\hline Grants & 2.7 & 2.7 & 3.0 & 2.7 \\
\hline Total expenditure & 29.9 & 27.1 & 27.4 & 26.1 \\
\hline Current spending & 18.4 & 17.6 & 18.9 & 18.0 \\
\hline Wages and salaries & 8.6 & 9.0 & 9.9 & 9.6 \\
\hline Goods and services & 2.4 & 2.5 & 2.3 & 2.1 \\
\hline Interest payments & 1.0 & 1.2 & 1.3 & 1.4 \\
\hline Current transfers & 6.4 & 4.9 & 5.5 & 4.9 \\
\hline Investment expenditure & 11.6 & 9.6 & 8.5 & 8.1 \\
\hline Overall balance & -7.8 & -4.9 & -3.0 & -3.0 \\
\hline Memo: security expenditure & 2.5 & 3.2 & 3.7 & - \\
\hline
\end{tabular}

\section{B. Enhancing Revenue Mobilization}

\section{Higher domestic revenue mobilization is essential to create fiscal space for priority} investment and social spending, as well as increased security spending. The implementation of new revenue measures contained in the 2019 budget is a step in the right direction. Nevertheless, work stoppages in the Ministry of Finance in 2018 and the first quarter of 2019 constrained the ability to fully mobilize the implied revenue leading to further accumulation of tax arrears, and the deferral of some tax reforms.

22. Staff encouraged the authorities to pursue the simplification and the computerization of tax declaration and payment procedures. The introduction of remote filing and payment for large enterprises has been a success and is a leap forward in simplifying taxpayer obligations and 
lightening management tasks. In addition, further modernization of IT systems of the tax directorate (e-syntax) foreseen for late 2019 would enable mobile payments and cash-payments at banks, the online request and issuance of documents, and a remote request for VAT re-imbursement. The dematerialization process should be extended to all government taxes and all categories of taxpayers.

\section{The authorities committed to continue efforts to increase domestic revenue mobilization by focusing on the following tax policy and administration measures:}

- $\quad$ Enhancing the implementation of the new revenue measures identified by the 2019 Budget, to improve their contribution to domestic revenue mobilization (MEFP \57-59).

- Improving the collections of arrears. Perform a thorough assessment of tax arrears, analyze them in terms of seniority and degree of collectability, and establish a more efficient strategy for clearing them especially those accumulated in 2018 and 2019. In conjunction with the treasury (DGTCP) and the budget (DGB) directorates, finalize an instruction that establishes the conditions, procedures and administrative and accounting consequences of accepting bad debts as accounting write-offs and oversee implementation (MEFP \60).

- $\quad$ Simplifying and further improving the procedure for refunding VAT credits by: (1) limiting the production of supporting documents (invoices, customs documents, etc.) to enterprises that generate a risk; (2) conducting off-site or on-site audits of enterprises that present an obvious risk; (3) enabling online VAT refund claims; (4) replenishing the escrow account through a levy on the customs VAT and domestic VAT; and (5) establishing a special account for VAT credit refunds that is funded with a portion of VAT revenues and produce quarterly reports on the operational management of this account (structural benchmark).

24. The authorities have made significant efforts to safeguard customs revenue following the end of the verification contract in August 2018, but further progress is needed to improve and sustain revenue collection. The creation of a Unit to manage and monitor the application of transaction value, the development of IT tools to make visit certificate mandatory, the establishment of products at risk list to enforce control are essential to ensure that the customs department is equipped and staffed to control the value of imports in line with WTO standards. Moving forward, the authorities are committed to advance the following custom administration reforms (MEFP \5859):

-

control of value. works machinery to CCVA. Côte d'Ivoire.

Increasing by 28 the number of imported products at higher risk of fraud subjected to the

Pursuing the outsourcing of the evaluation of used vehicles under ten years old and public

Finalizing the interconnection of Burkina Faso's customs IT systems with those of Togo and 
- $\quad$ Completing the splitting of the Bobo Station Customs Office (Bobo Inter) in three offices (road, railway station and hydrocarbon) to improve efficiency.

- $\quad$ Ensuring that the customs department is equipped and staffed to control the value of imports in line with WTO standards with the end of COTECNA's support.

- Strengthening the post-clearance audit so that it plays its decisive role in the system to take over full responsibility for transaction value.

\section{Managing Energy Subsidies}

25. Energy subsidies constitute a significant cost on the budget and weigh on the fiscal position. Recurrent delays in subsidy payments led to significant accumulation of state debt to SONABHY in the past. At end-June 2018, the total amount of unpaid subsidies stood at 176.9 billion CFAF (or 2.3 percent of GDP). An agreement between the government and SONABHY was signed in December 2018 which allowed the payment of 20 billion CFAF through direct transfers, and the securitization of the remaining amount in two separate sets of government bond notes of 60 billion CFAF and 96.9 billion CFAF. As the securitization agreement covered the stock of outstanding debt by end-June 2018, new debt incurred in the second half of 2018 put the stock of debt at 64.6 billion CFAF at end-2018. The 2019 budget provisioned 116 billion CFAF for energy subsidy: 50 billion CFAF for 2019 subsidies, and 66 billion CFAF to clear subsidy arrears accumulated in the second half of 2018.

26. In light of the budgetary burden from subsidies, the authorities adopted in November 2018 a new automatic fuel price adjustment mechanism to reduce the fiscal cost of fuel subsidy. Under the new mechanism, an upward fuel price adjustment was enforced in November 2018 (the first since early 2017), followed by a downward adjustment in January 2019. The updated fuel price structure translated into profits in SONABHY sales in January and February 2019. Despite losses in March 2019, SONABHY recorded 4.8 billion CFAF profits in the first quarter of 2019.

\section{The implementation of the new automatic fuel price adjustment mechanism needs to} be more consistent. Since the last adjustment in January, the authorities have not implemented the mechanism fully. The April adjustment did not take place as the authorities used discretion to keep prices unchanged. The next adjustment is expected to take place in July. Since the government has committed to cover any difference between the cost-recovery price and the retail price it imposed to SONABHY, the discretionary implementation of the mechanism could lead the subsidy to persist. Under the current implementation approach, the Prime Minister has to signoff each time to make the price adjustment effective. Going forward, the authorities have committed to strengthen their implementation approach (MEFP 180) by (i) delegating the enforcement of the mechanism from the Prime Minister (no signoff needed) to a technical team or a ministry, while keeping the discretion for the Prime Minister to intervene in exceptional circumstances and (ii) improving the government's communication on fuel prices by publishing the amounts of subsidy paid per unit of each fuel type, and through outreach campaigns with the local media on the budgetary trade-offs associated with high subsidies. The authorities indicated that the discretionary power of the Prime Minister is 
needed to reflect the limitations in using social safety nets to mitigate the potential impact of fuel price adjustments. Staff emphasized the importance of using this discretion judiciously and sparingly.

\section{Containing the Medium-Term Growth in the Wage Bill}

\section{The proliferation of sectoral salary scales with built-in comparability clauses has} created the conditions for continuous pay disputes. Sectoral wage negotiations have led to a complex compensation structure marked by a proliferation of sector-specific allowances. In 2018, the wage bill reached 9.0 percent of GDP (705 billion CFAF), an increase of 0.4 percentage points from 2017. Salaries are projected to further rise in 2019, reaching 9.9 percent of GDP (836 billion CFAF). Despite efforts to increase tax revenue in recent years, the wage bill has reached more than 50 percent of tax revenue in 2018 , significantly above the WAEMU convergence criterion of 35 percent of tax revenue.

29. The authorities' public sector pay strategy is based on an overarching legislation that would allow for a consolidation of civil service compensation based on unified public sector pay and allowance scales. This aims to remove continuous comparability cycles, eliminate inequity issues, and allow for more centralized collective bargaining going forward. A first draft organic law examined by the Cabinet defines the broad principles of pay and allowances that should govern the public service going forward. Among other things, this step would allow to (i) harmonize job descriptions based on minimum qualifications for functional groups, (ii) revise salary and indemnity scales, and (iii) update the salary indexation methodology. The adoption of the organic law requires a constitutional amendment, which can be done either through a referendum (an expensive option) or through Parliament (the government lacks the super majority of 80 percent required for this option).

\section{The authorities' 2020-2022 multiannual budget and economic programming} document projects a continuous reduction in the ratio of the wage bill to tax revenue ratio from its peak in 2019. This medium-term budget framework which was adopted by the Cabinet of Ministers in June 2019 (structural benchmark), will set the path for the country to lower its wage bill in order to reach the WAEMU convergence criterion of 35 percent of wage bill to tax revenue.

\section{Given difficulties in moving ahead with a constitutional reform, the authorities are} now focusing on a two-stage strategy to contain the wage bill. First, continue exploring politically feasible ways to adopt their reform package; second, in the near term, keep the wage bill in line with the understandings reached at the first review. A second conference will take place to seek further support of stakeholders for the reform package ${ }^{3}$. However, the process of migrating from individual pay scales to the harmonized system may risk introducing an additional burdensome increase that would undermine the objective of reducing the share of wages in the budget going forward.

\footnotetext{
3 The first conference, held in June 2018, discussed reform options for a medium-term public sector strategy (IMF Report No 19/15, EBS/18/125).
} 


\section{The authorities are taking the following measures to contain the wage bill in the meanwhile:}

- Organic law. The government will endeavor to have this law enacted as soon as possible.

- Communication. The authorities will make a commitment in the form of a public statement by the government regarding their strategy to contain the wage bill to tax revenue ratio and ensure its reduction starting in 2020 (prior action).

- Strategy document. A strategic document will be prepared by end-September 2019 that would set out the medium-term perspectives for pay, allowances, and recruitment planning consistent with achieving the WAEMU convergence criterion (MEFP आ79).

- Containing the wage bill to amounts previously agreed in the first review. This reflects the authorities' commitment to sign no new agreements that could increase the wage bill until the comprehensive reform package is in place (MEFP $\llbracket 79$ ).

- Administrative measures. New hires for 2018 and 2019 have been reduced by 33 and 44 percent respectively, compared to initial projections of 10,868 and 10,615 . To ensure a better match between needs and workers, a decree defining job descriptions of 14 ministries has been adopted, and similar work for the remaining ministries is in progress. An administrative census has been carried out to assess the adequacy between the number of positions in each region and the number of workers allocated to the regions by grade and ministry. The preliminary results are being used to reinvigorate the redeployment policy and to inform the new hiring strategy. A civil service census intended to support the audit of the payroll database is being prepared with the support of the World Bank. The ongoing functional review will also help to base recruitment and posting of public servants on pre-identified job vacancies (MEFP $\llbracket \uparrow 18,19$, and 79).

- Bonuses to Ministry of Finance staff. The 2019 budget limited the "Prime de Motivation" bonuses to 25 percent of base salary. This represented a significant loss in income for a number of staff, which risked a spate of personal loan foreclosures and some stress in the banking sector's risk-weighted assets at a time when banks are adjusting to more stringent supervision (Basel II) standards enforced since 2018. Following union action, an agreement was reached to replace these bonuses with a prime de motivation with an annual fiscal cost estimated at CFAF 23 billion. These bonuses are to be included in the budget from 2020 onward (MEFP Table 4).

\section{E. Enhancing the Efficiency of Public Spending}

33. Burkina Faso has successfully transitioned to program budgeting, but further steps are needed to finalize the process. Burkina Faso has started adopting program budgeting in 2017 and annual performance plans were produced in accordance with a structure aligned with good practices. However additional steps are needed to further rationalize expenditure procedures. In this regard, the publication of the budget on a functional basis is a priority. The program budget is based on a functional classification of expenditure which allows a clear overview of priorities. In the 
standard budget documentation, however, the budget is not presented on this basis. Including this information in the documentation would give decision-makers and the public a better idea of how the budget reflects policy priorities. There is also a need for strengthening the legal and institutional framework for public investment management and PPPs.

\section{Implementing the treasury single account (TSA) would help to optimize cash} management and reduce borrowing needs. The authorities are proceeding with a stock taking of all accounts in commercial banks with a view to closing inactive accounts and consolidating their balances in the TSA system. At the same time, the authorities are elaborating a decree to delimit the scope of the TSA and its modalities of operation. The technical support for setting up the TSA (connection to STAR and SICA through SWIFT) is being acquired with the support of the World Bank.

\section{Timely and reliable financial accounts are a pre-requisite for the successful transition} to the TSA. This is essential to reconcile the general ledger with the statements of accounts transmitted by the BCEAO and commercial banks. At present, the general ledger account is only produced at year end which limits the capacity to rationalize borrowing and expenditure plans during the course of the year. To this end the authorities are moving progressively towards the production of monthly accounts. In parallel, the authorities are also moving their accounting system towards full accruals accounting which is due to be completed by the end of 2019 .

\section{The public financial management (PFM) reforms mentioned above require stronger} coordination. It would be useful to establish a unit within the Treasury directorate to oversee the implementation of the TSA. For program budgeting, it is important among other things, to implement the program management structure by appointing program managers at various levels and move toward an integrated, non-modular information system across various revenue directorates.

\section{F. Social Sector Spending}

37. Key social indicators lag behind regional comparators (Text Figure 2). Adult literacy and child mortality are among the worst in sub-Saharan Africa while access to health care is extremely limited. The existing social safety net is not sufficiently adapted to fully mitigate the potential impacts of shocks and policies on the poor.

\section{The expansion of coverage and the rationalization of the delivery of social safety nets} are an urgent necessity. Social safety net programs cover less than 3 percent of the population with only about one third based on cash transfers. At the same time, the targeting of social safety net programs is not well aligned with poverty indicators ${ }^{4}$. The authorities are investigating how to compensate vulnerable groups for rises in fuel prices but have yet to identify an appropriate mechanism. Staff urged the authorities to protect social spending in line with the program indicative floor on poverty-reducing current social expenditure. The authorities' immediate

\footnotetext{
${ }^{4}$ The Way Forward for Social Safety Nets in Burkina Faso, World Bank, 2019.
} 
objectives in this area include promoting health insurance for all and ensuring the effective implementation of free healthcare for pregnant women and children under five.

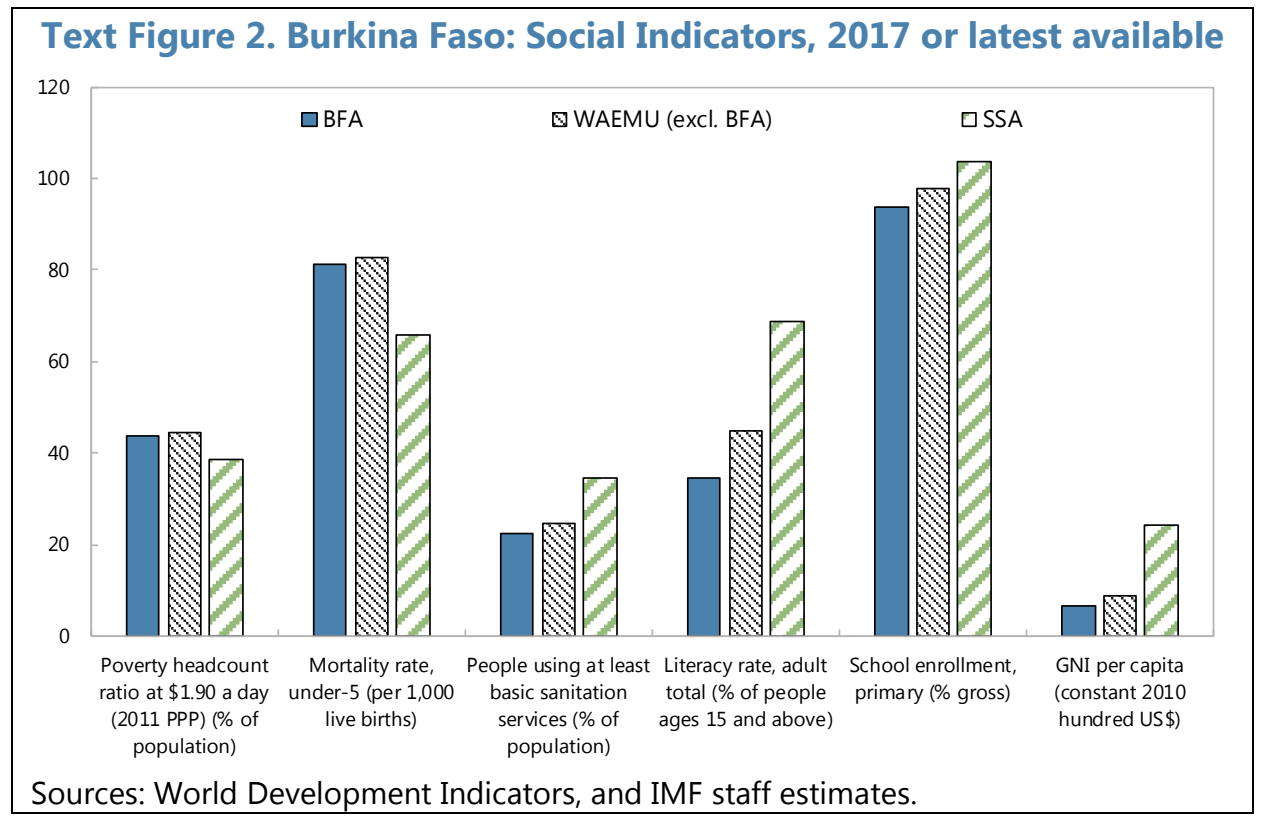

\section{G. Governance}

39. Governance reforms, including effort to implement program commitment on assets declaration, should be sustained. The government remains committed to strengthen the legal and institutional framework that are important for the effectiveness of the High Authority for State Control and Anti-Corruption (ASCE-LC). The implementing regulations of the anti-corruption Law No. 004-2015/CNT of March 3, 2015 are currently in cabinet for consideration. The 2017 audit report on government management has been concluded and published, and the 2018 report is in preparation. The reform to automating the Declaration of Interest and Assets (DIP) by the individuals covered by the 2015 law, including public servants, has faced delays but is progressing. The development of a procedures manual for the DIP has been completed. However, uncertainty remains regarding the hiring of an external expert to implement the DIP platform and the related IT procurement. Thus, while the ASCE-LC website has been created, its envisaged DIP platform is not operational. The DIP platform is expected to be operational by March 2020 (structural benchmark). The ASCE-LC also envisages to develop a national strategy of corruption prevention. Peer-learning missions to Côte D'Ivoire and Morocco are planned to collect international experience in this area.

\section{PROGRAM MONITORING AND OTHER ISSUES}

40. Staff support the authorities' request for a waiver for non-observance of the ceiling on net domestic financing driven by cash adjustment. The authorities have taken corrective measures and are planning further consultations with the Resident Experts of the IMF on matters of PFM. Proposals for revised June and December 2019 program targets, as well as newly proposed structural benchmarks are presented in MEFP. In terms of measures to contain the public sector 
wage bill, the authorities also committed to submit to the national assembly by end-September 2019 a draft 2020 budget that is aligned with the current framework, especially with respect to the public wage bill.

41. Completion of the review will release a third disbursement under the ECF arrangement in the amount of SDR $\mathbf{1 8 . 0 6}$ million, in line with the disbursement schedule approved at the outset of the arrangement (Table 7). Quantitative performance criteria (PC) and indicative targets (IT) through June 2020 are set out in Tables 1 and 3 of the MEFP. The structural benchmarks to support the program's objectives are listed in Tables 2 and 4 in the MEFP.

42. The program is fully financed (Table 6). Financing assurances are in place for the next 12 months of the program and there are good prospects of financing for the remainder of the program. The external financing requirement is met through budget support and project loans from other IFIs and bilateral donors (totaling 412 billion CFAF), as well as limited non-concessional borrowing. While project support (grants and loans) are projected to remain robust over the program period, direct budget support (loans and grants) are projected to continue a downward trend.

43. Burkina Faso's capacity to repay the Fund remains adequate (Table 8). Obligations to the Fund would peak in 2019 at only 1.1 percent of government revenue or 0.2 percent of GDP.

44. Capacity Development. Staff and the authorities agreed on technical assistance priorities under the capacity building framework. The authorities have been receiving extensive technical assistance in the agreed areas, including mobilization of domestic revenues (customs and taxation), management of fiscal risks, control of re-current spending, particularly on wages and salaries, and reforms to strengthen spending efficiency, especially regarding public investment spending, public financial management, and debt management. Further technical assistance to reinforce capacity in these areas and facilitate implementation of recommendations is expected to continue.

45. Safeguards assessments. The update safeguards assessment of the BCEAO completed in 2018 found that the central bank had maintained a strong control environment, audit arrangements were in broad conformity with international standards, and the financial statements were prepared in accordance with International Financial Reporting Standards (IFRS). The BCEAO has recently enhanced the oversight role of its audit committee in line with the recommendations of the assessment.

\section{STAFF APPRAISAL}

46. Burkina Faso continues to pursue its development goals in the face of increasing challenges from security threats and public sector pay disputes. The recent review of the implementation of the Programme Nationale de Development Economique et Sociale (PNDES) noted progress in key priorities, in infrastructure provision and social services. At the same time, however, the review acknowledges that the scope to increase fiscal space for development priorities is constrained by the need to devote resources to security and the size of the public sector pay bill. 
47. Economic activity remains resilient in the face of increased security and social tension pressures. Security incidents have increased and raised the security-related expenditure needs. Strikes or industrial actions by various groups of civil servants have disturbed the functioning of public administration. The impacts of these pressures have been limited thus far but could affect medium-and-long-term prospects. GDP growth reached 6.8 percent in 2018 and is projected to stabilize around 6 percent in 2019 and in the medium term, as the impact of the bumper harvest of 2018 fades away.

48. Program performance remains broadly satisfactory. All quantitative targets but one, and most structural benchmarks have been met. The authorities have taken corrective actions to address the missed quantitative target, and staff support the proposals for revised program targets through end-December. Eight out of thirteen structural benchmarks have been met. Effort is underway to meet those that were not met.

\section{The authorities' commitment to the fiscal deficit of $\mathbf{3}$ percent of GDP in $\mathbf{2 0 1 9}$ is} welcome. In the light of the windfall revenues of about 1.4 percent of GDP from the 4G license proceeds in 2019, there is adequate fiscal space to allow some increase in investment and security spending without relaxing borrowing targets. Windfall revenues should only be used after the passage of the Loi de Finance Rectificative. Looking forward, staff encouraged the authorities not to rely on one-off revenues to accommodate permanent expenditures. Moreover, staff urged the authorities to continue to focus on efforts to increase fiscal space through broadening the tax base, improved tax administration, and bearing down on exemptions. At the same, staff reiterated the importance of enhancing the efficiency of expenditure by following the recommendations on investment spending contained in the PIMA.

50. The political uncertainty surrounding the adoption of the organic law is disappointing, but staff welcome the first steps taken by the authorities to control the wage bill and urge them to remain committed to the agreed transitional measures, while pursuing a durable solution. Delays in proceeding with the long-planned revision to the constitution have held back the process of reform. Staff urged the authorities to provide further details on the up-front cost of the reforms and their implications going forward. Given the uncertainty surrounding the adoption of the Organic Law in the near term, staff and authorities agreed that in the meanwhile, the nominal wage bill will remain in line with the projections agreed at the last review. At the same time, the authorities are committed to eschewing any new pay awards until the reform package is in place. The authorities would use this time to build consensus around the appropriate level of public pay, through the articulation of its strategy to the wider public.

\section{The authorities agreed to reinforce the implementation of the automatic fuel price} adjustment mechanism while developing social safety nets for the most vulnerable. It is critical that the adjustment be made regularly and consistently. However, the discretion granted to the Prime Minister to overrule the decisions of the technical committee in exceptional circumstances should be used judiciously and be phased out as social safety nets improve. The new price adjustment mechanism would be strengthened by complementing it with greater transparency through improved communication and avoiding discretion in its implementation. Measures so far 
taken by the authorities to limit the negative impacts of the fuel price adjustment on the poor comprise the temporary agreement reached with transport operators to keep fares. The authorities committed to entrust the implementation of the price adjustment mechanism to a technical team which is not subject to political considerations to ensure its enforcement.

52. Reforms in public financial management (PFM) require stronger coordination. The complex nature of key reform priorities in the area of program budgeting, the move towards accruals accounting, ensuring more timely accounts, and implementing the TSA require intensive cooperation between departments within the Ministry of Finance and other institutions.

53. Staff supports the authorities' request for a waiver and recommend the completion of the second review and the disbursement of SDR18.06 million under the ECF. The authorities' letter of intention and MEFP spell out measures necessary to ensure that the objectives of the program would be met. The capacity to repay the Fund remain adequate and risks to the program are manageable. 
Figure 1. Burkina Faso: Recent Economic Developments

Growth accelerated to 6.8 percent in 2018, despite rising security concerns

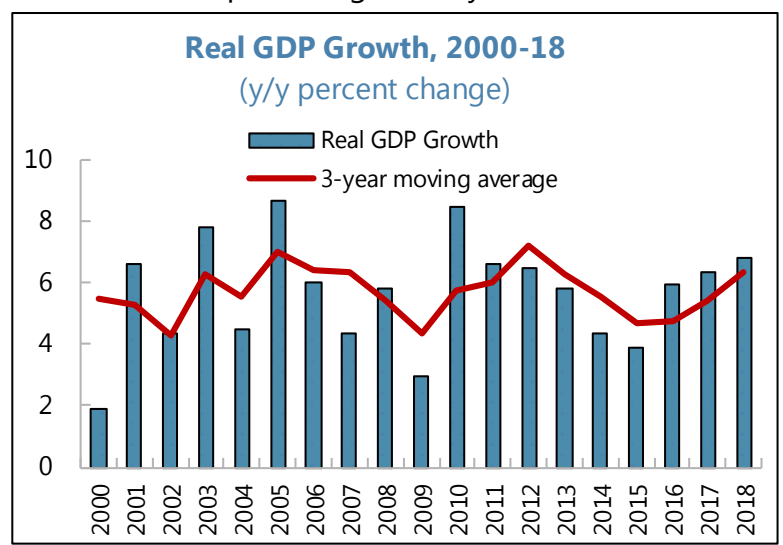

Credit growth slowed down sharply in 2018 amid more stingent prudential rules.

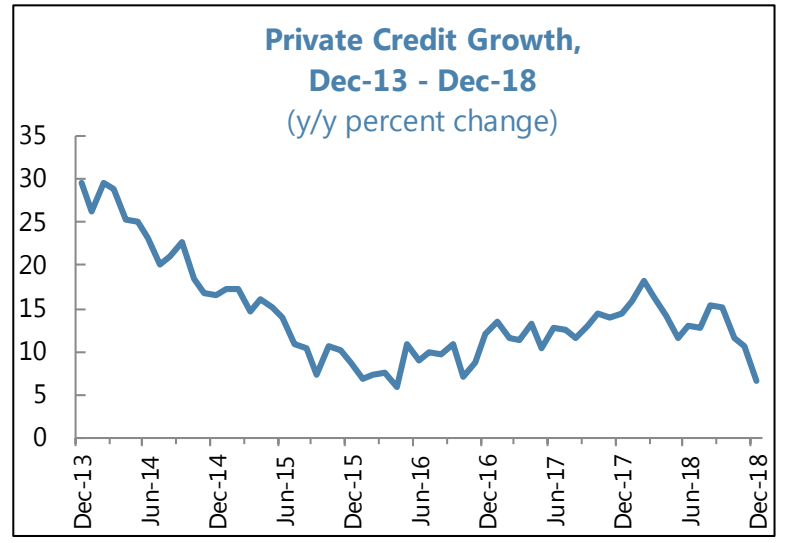

Tax revenues remained stable in 2018, despite a weak perfomance in the first semester.

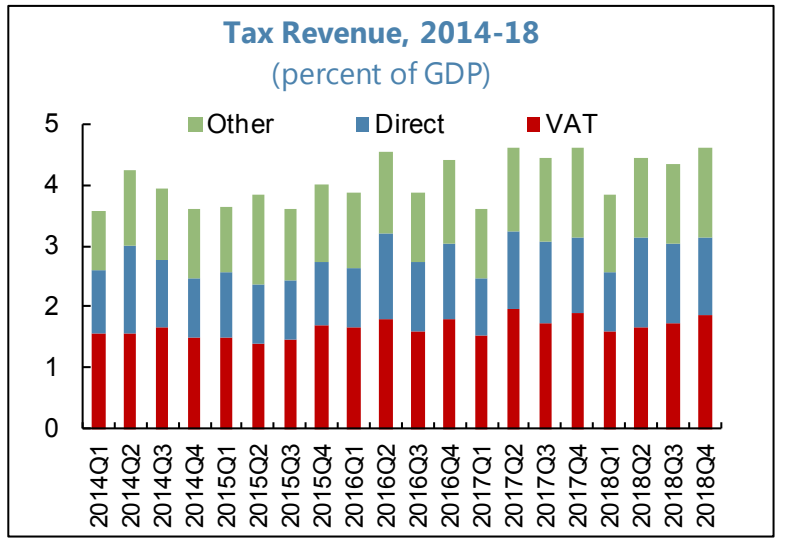

...supported by agriculture, services and mining.

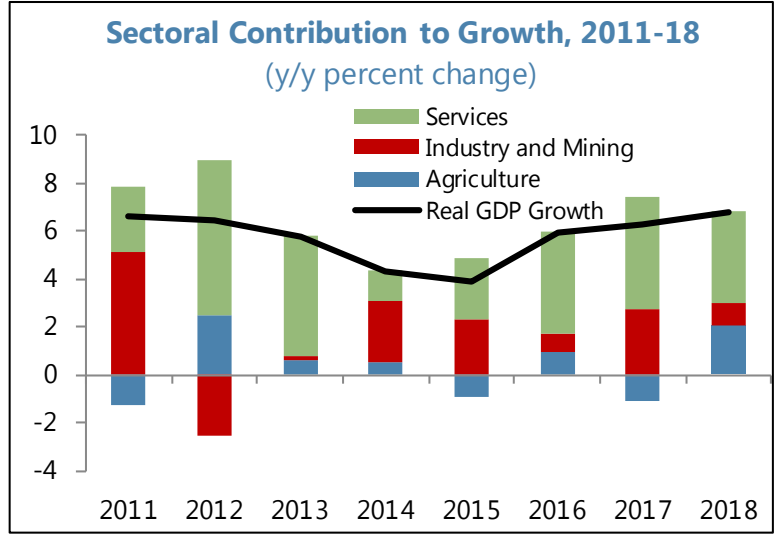

Inflation remained subdued, despite the fuel price hikes in late 2018.

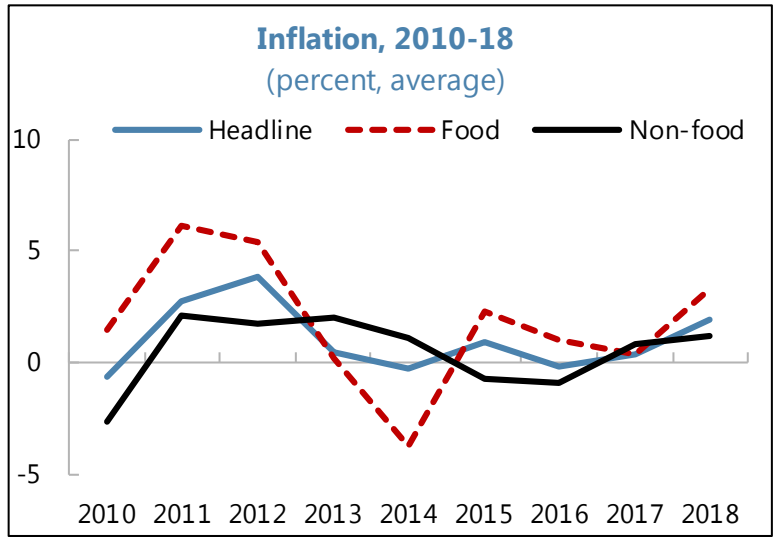

....and public investment execution increased, despite declining as a percentage of GDP.

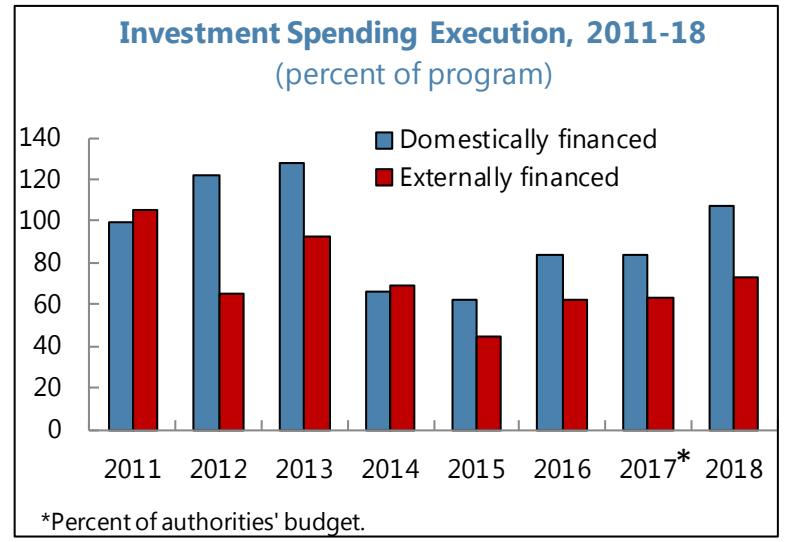

Source: Burkinabè authorities; and IMF staff calculations. 
Figure 2. Burkina Faso: Real and External Developments

Gold production and export volumes reach a new high as the cotton sector faces a crisis of production

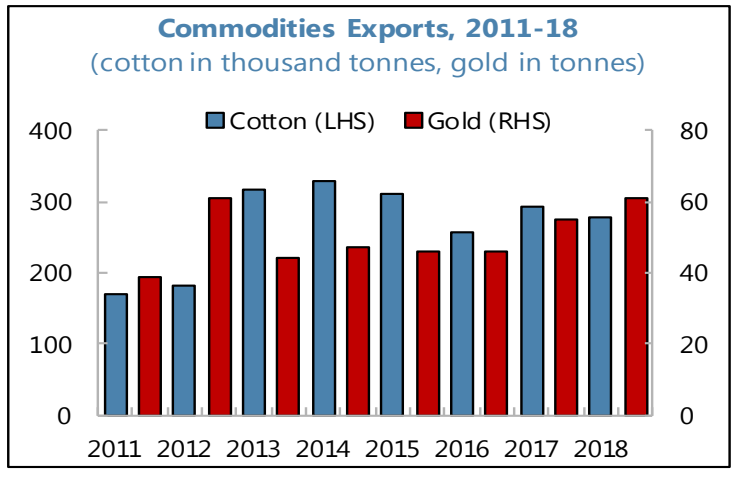

The gold mining sector is increasingly the main driver of total exports

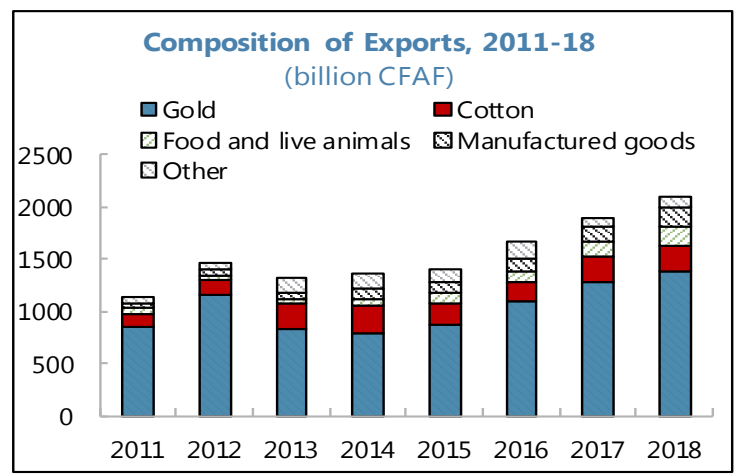

The current account balance showed a strong improvement in 2018 at $-5.8 \%$ of total GDP

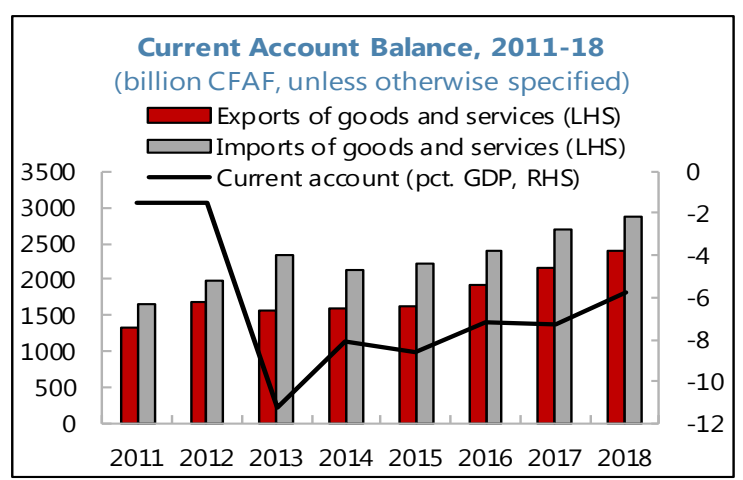

...while higher average cotton prices in 2018 represented a buffer to cotton exports.

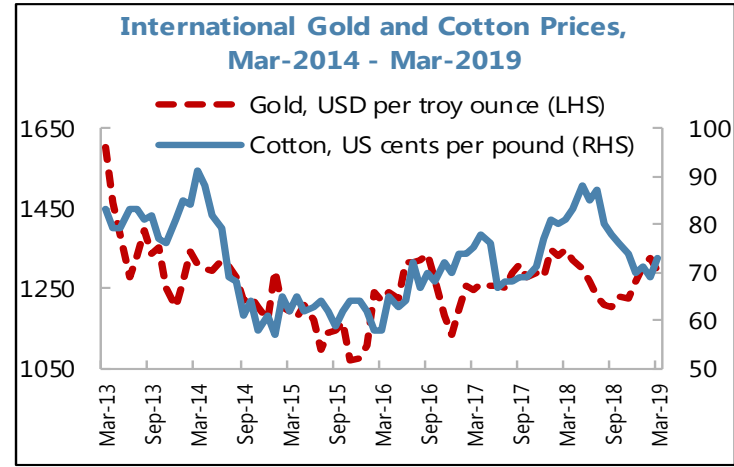

...while imports are mostly driven by oil and manufactured goods.

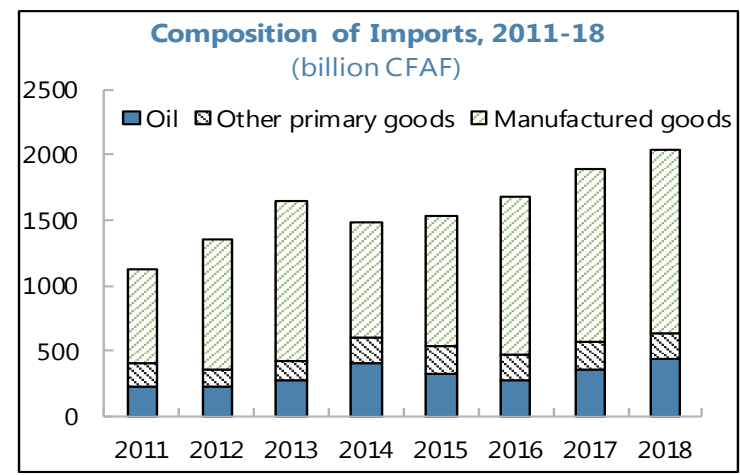

The real exchange rate depreciated by end of 2018 , after the appreciation in the start of the year

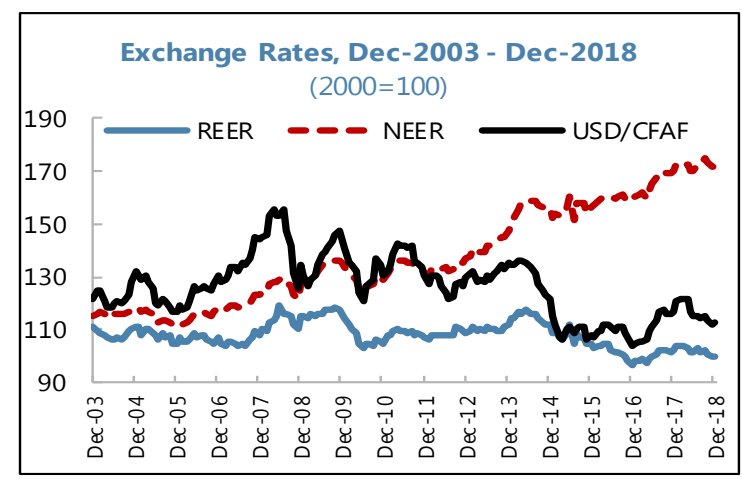

Sources: Burkinabè authorities; and IMF staff calculations.

Note: Figures for 2018 are preliminary. 
Figure 3. Burkina Faso: Fiscal Developments

Domestic revenue remained stable in 2018 .

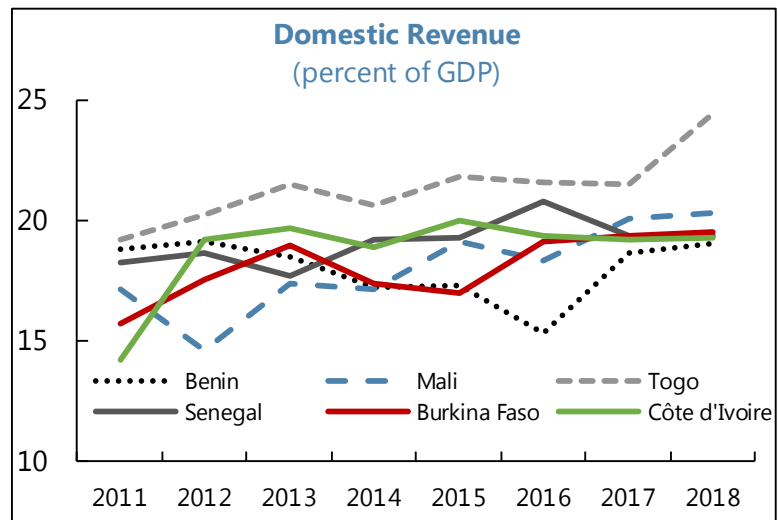

The wage bill is higher than in peers.

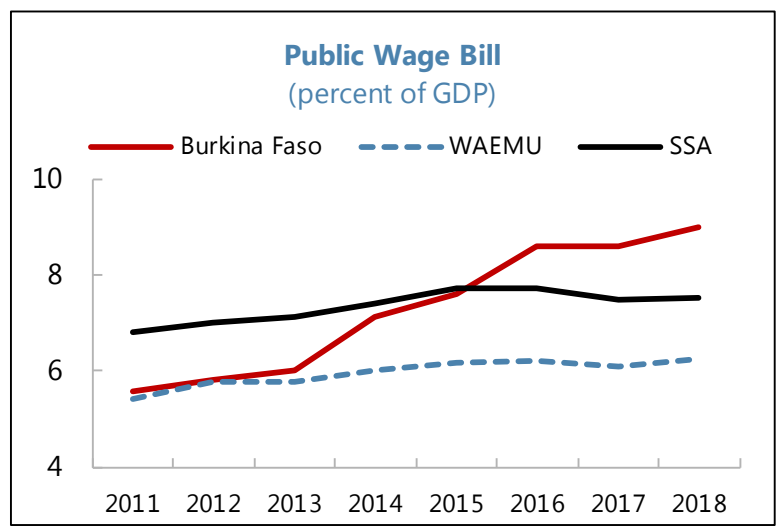

The fiscal deficit shrinked markedly...

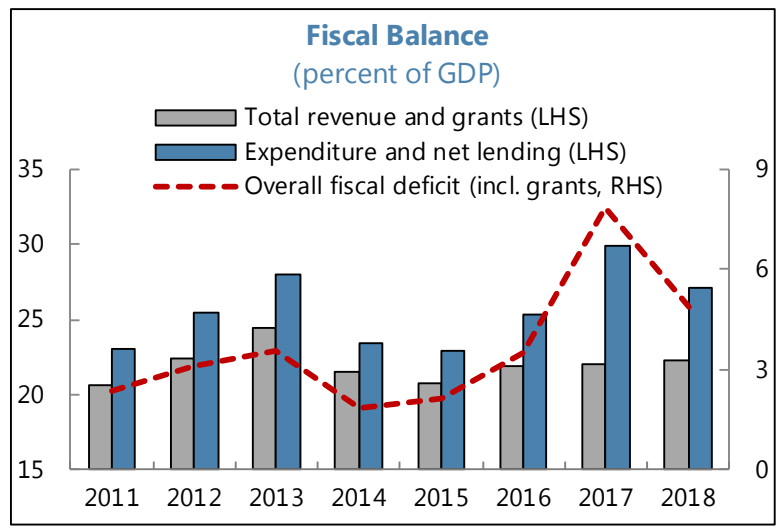

Current spending declined.

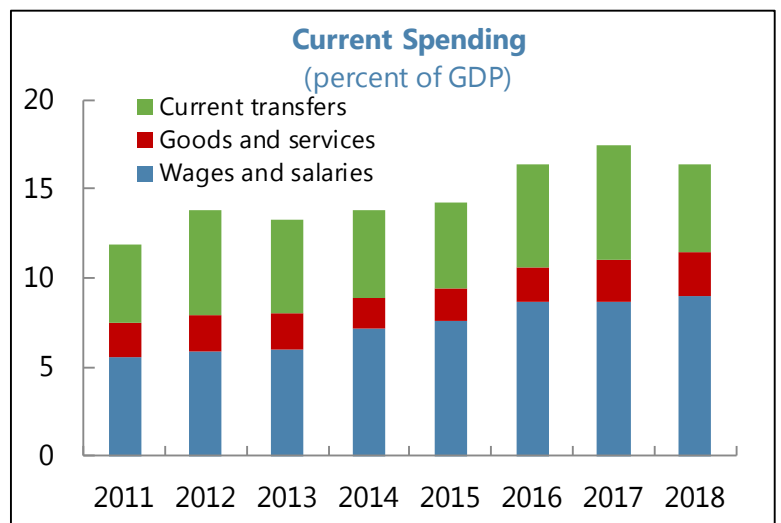

Investment spending is estimated to have declined in 2018.

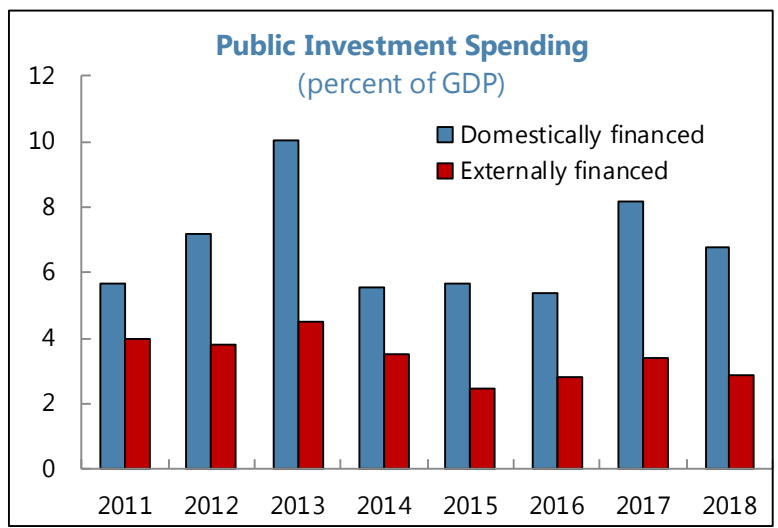

...although public debt increased as the share of domestic debt continued to rise.

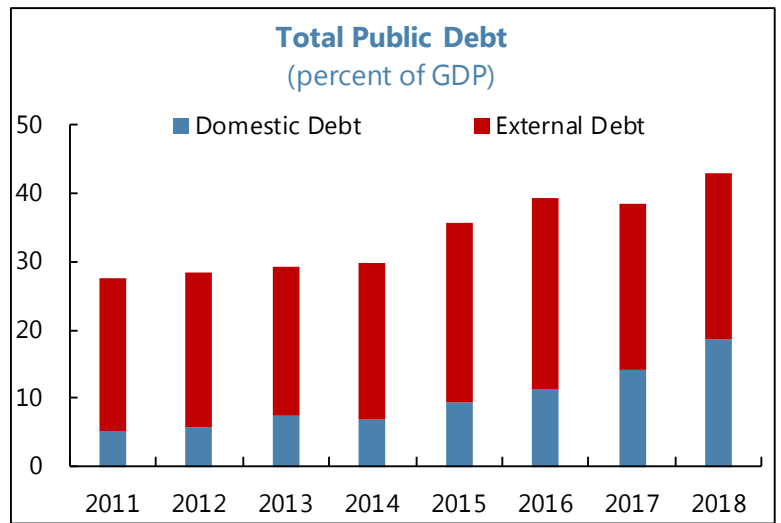

Sources: Burkinabè authorities; and IMF staff calculations. 
Table 1. Burkina Faso: Selected Economic and Financial Indicators, 2016-24

GDP and prices

GDP at constant prices

GDP deflator

Consumer prices (annual average)

Consumer prices (end of period)

\begin{tabular}{|c|c|c|c|c|c|c|c|c|}
\hline 2016 & 2017 & 2018 & 2019 & 2020 & 2021 & 2022 & 2023 & 2024 \\
\hline Act. & Est. & Prel. & Proj. & Proj. & Proj. & Proj. & Proj. & Proj. \\
\hline
\end{tabular}

(Annual percentage change, unless otherwise indicated)

\section{Money and credit}

Net domestic assets (banking system) 1/

Credit to the government (banking system) $1 /$

Credit to Private Sector

Broad money (M3)

Private sector credit/GDP

$\begin{array}{rllllllll}5.9 & 6.3 & 6.8 & 6.0 & 6.0 & 6.0 & 6.0 & 6.0 & 6.0 \\ -1.3 & 4.8 & 2.4 & 2.0 & 2.0 & 2.0 & 2.0 & 2.0 & 2.0 \\ -0.2 & 0.4 & 2.0 & 1.1 & 1.4 & 2.0 & 2.0 & 2.0 & 2.0 \\ -1.6 & 2.1 & 0.3 & 2.0 & 2.0 & 2.0 & 2.0 & 2.0 & 2.0\end{array}$

\section{External sector}

Exports (f.o.b.; valued in CFA francs)

Imports (f.o.b.; valued in CFA francs)

Current account (percent of GDP)

Real effective exchange rate

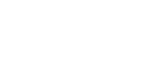

\section{Central government finances}

Of which: tax revenue

Total expenditure and net lending

Of which: current expenditure

$-1.3 \quad 11.4$

$-4.5 \quad 5.9$

$12.1 \quad 14.4$

$11.8 \quad 21.6$

$30.5 \quad 31.3$

$\begin{array}{ccc}8.6 & 12.1 & 13.6 \\ -0.2 & 5.1 & 4.9 \\ 6.7 & 12.7 & 12.9 \\ 10.4 & 13.6 & 15.4 \\ 30.5 & 31.8 & 33.2\end{array}$

$\begin{array}{ccccccccc}19.9 & 12.6 & 10.8 & 5.9 & 8.9 & 7.6 & 6.0 & 6.2 & 6.1 \\ 8.8 & 12.7 & 7.9 & 7.5 & 6.1 & 6.4 & 6.5 & 6.8 & 6.9 \\ -7.2 & -7.3 & -5.8 & -6.1 & -5.3 & -4.7 & -4.6 & -4.5 & -4.5 \\ -5.3 & -1.5 & 1.9 & \ldots & \ldots & \ldots & \ldots & \ldots & \ldots\end{array}$

(Percent of GDP, unless otherwise indicated)

Overall fiscal balance, incl. grants (commitments) Total Public Debt

Of which: external debt

$\begin{array}{lllllllll}19.1 & 19.4 & 19.5 & 21.3 & 20.4 & 20.8 & 21.2 & 21.5 & 21.8 \\ 16.7 & 17.3 & 17.3 & 17.8 & 18.3 & 18.7 & 19.0 & 19.3 & 19.6 \\ 25.4 & 29.9 & 27.1 & 27.4 & 26.1 & 26.4 & 26.7 & 26.9 & 27.2 \\ 17.4 & 18.4 & 17.6 & 18.9 & 18.0 & 17.4 & 17.3 & 16.9 & 16.9 \\ -3.5 & -7.8 & -4.9 & -3.0 & -3.0 & -3.0 & -3.0 & -3.0 & -3.0 \\ 39.2 & 38.4 & 42.9 & 42.5 & 42.2 & 41.9 & 41.7 & 41.5 & 41.3 \\ 27.9 & 24.1 & 24.3 & 23.1 & 22.3 & 21.2 & 20.6 & 20.1 & 19.6\end{array}$

Memorandum items:

Nominal GDP (CFAF billion)

6,444

7,177
644

7,849
726

8,484

9,170

9,916

$10,723 \quad 11,593 \quad 12,535$

Nominal GDP per capita (US\$)

$583 \quad 644$

740

787

833

883

933990

Sources: Burkinabè authorities; and IMF staff estimates and projections.

$1 /$ Percent of beginning-of-period broad money. 


\begin{tabular}{|c|c|c|c|c|c|c|c|c|c|}
\hline \multicolumn{10}{|c|}{ Table 2. Burkina Faso: Balance of Payments, 2016-24 } \\
\hline \multirow[b]{4}{*}{ Current account } & 2016 & 2017 & 2018 & 2019 & 2020 & 2021 & 2022 & 2023 & 2024 \\
\hline & Act. & Est. & Prel. & Proj. & Proj. & Proj. & Proj. & Proj. & Proj. \\
\hline & \multicolumn{9}{|c|}{ (CFAF billions, unless otherwise indicated) } \\
\hline & -462 & -520 & -454 & -518 & -486 & -471 & -498 & -526 & -564 \\
\hline Trade balance & 0 & -3 & 51 & 23 & 87 & 122 & 117 & 108 & 92 \\
\hline Exports of goods & 1,676 & 1,887 & 2,090 & 2,214 & 2,412 & 2,596 & 2,752 & 2,924 & 3,104 \\
\hline Of which: cotton & 198 & 253 & 250 & 201 & 245 & 251 & 261 & 273 & 285 \\
\hline Of which: gold & 1,092 & 1,278 & 1,387 & 1,536 & 1,672 & 1,830 & 1,954 & 2,090 & 2,233 \\
\hline Imports of goods & $-1,677$ & $-1,890$ & $-2,039$ & $-2,191$ & $-2,326$ & $-2,474$ & $-2,636$ & $-2,816$ & $-3,012$ \\
\hline Of which: oil & -283 & -356 & -444 & -421 & -440 & -457 & -477 & -504 & -533 \\
\hline Services, net & -471 & -518 & -522 & -538 & -554 & -580 & -607 & -635 & -664 \\
\hline Primary income, net & -232 & -230 & -231 & -246 & -252 & -258 & -269 & -275 & -283 \\
\hline Secondary income, net & 241 & 232 & 249 & 242 & 233 & 245 & 261 & 277 & 291 \\
\hline Of which: Official transfers, net & 111 & 100 & 110 & 97 & 80 & 86 & 93 & 100 & 108 \\
\hline Capital account & 150 & 173 & 176 & 218 & 221 & 221 & 229 & 238 & 246 \\
\hline Project grants & 99 & 120 & 122 & 164 & 167 & 167 & 176 & 184 & 193 \\
\hline Financial account & 245 & 477 & 585 & 336 & 325 & 347 & 380 & 398 & 448 \\
\hline Direct investment & 202 & -4 & -5 & -11 & -5 & 35 & 2 & 3 & 5 \\
\hline Portfolio investment & -236 & 70 & 247 & 94 & 89 & 108 & 108 & 107 & 107 \\
\hline Other investment & 279 & 412 & 342 & 253 & 240 & 204 & 270 & 289 & 336 \\
\hline Commercial banks & -66 & -79 & 69 & -15 & -14 & -6 & -6 & -6 & -6 \\
\hline General government & 145 & 77 & 76 & 78 & 84 & 90 & 129 & 144 & 160 \\
\hline Project loans & 82 & 124 & 103 & 131 & 125 & 125 & 141 & 159 & 180 \\
\hline Program loans & 63 & 0 & 28 & 20 & 15 & 27 & 52 & 56 & 60 \\
\hline Amortization of public loans (excl. IMF) & -39 & -50 & -55 & -72 & -56 & -61 & -64 & -72 & -81 \\
\hline Other private & 200 & 414 & 197 & 190 & 171 & 119 & 147 & 151 & 182 \\
\hline Errors and omissions & -3 & -4 & 5 & 0 & 0 & 0 & 0 & 0 & 0 \\
\hline Overall balance & -71 & 125 & 311 & 36 & 60 & 97 & 110 & 110 & 130 \\
\hline Net change in foreign assets of the central bank & 71 & -125 & -311 & -36 & -60 & -97 & -110 & -110 & -130 \\
\hline of which: IMF net financing & -7 & 11 & 5 & -25 & -13 & 17 & 15 & 12 & 13 \\
\hline Disbursements (past and prospective) & 19 & 4 & 14 & 43 & 29 & 0 & 0 & 0 & 0 \\
\hline Repayments (excluding charges) & -12 & -14 & -19 & -18 & -16 & -17 & -15 & -12 & -13 \\
\hline Financing Gap & 0 & 0 & 0 & 0 & 0 & 0 & 0 & 0 & 0 \\
\hline & & & (Percen & $f G D P$, un & ss otherwi & e indicated & & & \\
\hline Memorandum items: & & & & & & & & & \\
\hline Exports of goods & 26.0 & 26.3 & 26.6 & 26.1 & 26.3 & 26.2 & 25.7 & 25.2 & 24.8 \\
\hline Imports of goods & -26.0 & -26.3 & -26.0 & -25.8 & -25.4 & -24.9 & -24.6 & -24.3 & -24.0 \\
\hline Trade balance (goods) & 0.0 & 0.0 & 0.7 & 0.3 & 0.9 & 1.2 & 1.1 & 0.9 & 0.7 \\
\hline Trade balance (services) & -7.3 & -7.2 & -6.7 & -6.3 & -6.0 & -5.8 & -5.7 & -5.5 & -5.3 \\
\hline Trade balance (goods and services) & -7.3 & -7.3 & -6.0 & -6.1 & -5.1 & -4.6 & -4.6 & -4.5 & -4.6 \\
\hline Current account $(-=$ deficit $)$ & -7.2 & -7.3 & -5.8 & -6.1 & -5.3 & -4.7 & -4.6 & -4.5 & -4.5 \\
\hline GDP at current prices (CFAF billions) & 6,444 & 7,177 & 7,849 & 8,484 & 9,170 & 9,916 & 10,723 & 11,593 & 12,535 \\
\hline BCEAO Reserves & & & & & & & & & \\
\hline In billion USD & 10 & 13 & 15 & 16 & 17 & 19 & 21 & 24 & 30 \\
\hline In months of next year's WAEMU imports & 3.9 & 4.1 & 4.4 & 4.4 & 4.2 & 4.4 & 4.6 & 4.9 & 5.3 \\
\hline In percent of broad money & 37.7 & 38.7 & 39.7 & 37.5 & 34.9 & 35.1 & 34.5 & 35.8 & 39.0 \\
\hline
\end{tabular}


Table 3. Burkina Faso: Monetary Survey, 2016-20

\begin{tabular}{|c|c|c|c|c|}
\hline 2016 & 2017 & 2018 & 2019 & 2020 \\
\hline Act. & Act. & Act. & Proj. & Proj. \\
\hline
\end{tabular}

Net foreign assets

BCEAO 1/

Assets

Liabilities

Commercial banks

Net domestic assets

Domestic credit

Net Bank credit to government

BCEAO

Commercial banks

Credit to other sectors

of which: Credit to private sector

Other items (net)

Shares and other equities

\section{Total broad money liabilities}

Liquid liabilities

Non-liquid liabilites (excl. from broad money)

(CFAF billions, unless otherwise indicated)

$\begin{array}{rrrrr}1,051 & 1,334 & 1,442 & 1,519 & 1,619 \\ -139 & 3 & 309 & 344 & 404 \\ 620 & 902 & 928 & 1,062 & 1,225 \\ 758 & 899 & 619 & 718 & 821 \\ 1,189 & 1,331 & 1,133 & 1,175 & 1,215 \\ & & & & \\ 1,650 & 1,947 & 2,220 & 2,644 & 3,187 \\ 2,064 & 2,444 & 2,731 & 3,216 & 3,759 \\ -80 & 74 & 67 & 246 & 442 \\ -142 & -48 & -57 & -32 & -18 \\ 61 & 122 & 124 & 278 & 460 \\ 2,144 & 2,370 & 2,665 & 2,970 & 3,318 \\ 1,963 & 2,246 & 2,396 & 2,701 & 3,048 \\ 18 & 33 & 40 & 101 & 101 \\ 396 & 464 & 471 & 471 & 471 \\ & & & & \\ 2,700 & 3,282 & 3,662 & 4,163 & 4,806 \\ 2,610 & 3,174 & 3,503 & 3,981 & 4,596 \\ 91 & 108 & 159 & 182 & 210\end{array}$

(Annual percentage change, unless otherwise indicated)

\section{Memorandum items:}

Net foreign assets

Net domestic assets 2/

Net credit to government 2/

Credit to Private Sector

Private sector credit (percentage of GDP)

Money supply

Velocity of money (GDP/M2) 3/
42.5

$-1.3$

$-4.5$

12.1

30.5

11.8

2.5
27.0

11.4

5.9

14.4

31.3

21.6

2.3
8.1

8.6

$-0.2$

6.7

30.5

10.4

2.2
5.3

12.1

5.1

12.7

$31.8 \quad 33.2$

$13.6 \quad 15.4$

$2.1 \quad 2.0$

Sources: Burkinabè authorities; and IMF staff estimates and projections.

$1 /$ Only includes current reserves in BCEAO Ouagadougou. It does not include all regional reserves.

2/ Annual change as a percentage of broad money from 12 months earlier.

3/ End-of-period average. 
Table 4. Burkina Faso: Selected Financial Soundness Indicators, 2012-June 2018

(in percent)

Capital Adequacy

Dec-12 Dec-13 Dec-14 Dec-15 Dec-16 Dec-17 Jun-18 1/

Reg. capital to risk-weighted assets

$\begin{array}{lllllll}12.4 & 10.2 & 11.4 & 11.1 & 13.0 & 12.2 & 11.8\end{array}$

\section{Asset Quality}

Gross NPLs / Total loans

Provisions / NPLs

$\begin{array}{rrrrrrr}10.3 & 9.9 & 8.6 & 8.9 & 8.9 & 8.8 & 8.7\end{array}$

\section{Loan Concentration}

5 largest clients / equity

By Sector: (share of total)

Agriculture

Extractive Industries

Manufacturing

Electricity, gas, water

Buildings/Public Works

Commercial (restaurants, hotels)

Transportation/communication

Insurance, real estate, business services

Other

67.7

62.6

64.9

67.6

70.4

66.4

69.9

$\begin{array}{lllllll}157.9 & 108.4 & 158.4 & 179.6 & 114.5 & 79.8 & 60.7\end{array}$

$\begin{array}{lllllll}1.8 & 3.3 & 2.8 & 1.9 & 3.7 & 4.4 & 5.5\end{array}$

$\begin{array}{lllllll}1.0 & 1.1 & 2.0 & 2.3 & 1.5 & 2.0 & 2.1\end{array}$

$\begin{array}{lllllll}9.8 & 13.9 & 16.1 & 15.2 & 13.2 & 13.6 & 11.6\end{array}$

$\begin{array}{lllllll}1.9 & 1.3 & 1.3 & 1.0 & 0.8 & 1.2 & 0.8\end{array}$

$\begin{array}{lllllll}13.2 & 14.6 & 13.6 & 16.6 & 16.2 & 16.9 & 17.1\end{array}$

$\begin{array}{lllllll}38.3 & 33.2 & 26.0 & 28.3 & 25.6 & 23.9 & 21.9\end{array}$

$\begin{array}{lllllll}9.6 & 11.7 & 9.5 & 8.4 & 10.3 & 8.8 & 9.6\end{array}$

$\begin{array}{lllllll}3.9 & 3.4 & 3.7 & 3.0 & 5.0 & 5.6 & 6.1\end{array}$

Liquidity

Loans to deposits

20.6

17.5

25.1

23.1

$23.7 \quad 23.7$

25.3

Liquid assets / total assets

$\begin{array}{lllllll}82.1 & 87.7 & 99.8 & 91.2 & 86.1 & 83.9 & 82.4 \\ 34.8 & 34.7 & 34.8 & 29.2 & 23.2 & 24.7 & 26.5\end{array}$

Sources: Central bank (BCEAO) authorities and IMF staff estimates.

1 / First year of data reporting in accordance with Basel II/III prudential standards and the new banking chart of account (interim data). 
Table 5a. Burkina Faso: Consolidated Operations of the Central Government, 2017-24

(CFAF billions)

\begin{tabular}{|c|c|c|c|c|c|c|c|c|c|c|}
\hline Total revenue and grants & 1,584 & 1,795 & 1,746 & 1,937 & 2,069 & 2,116 & 2,316 & 2,538 & 2,771 & 3,024 \\
\hline Total revenue & 1,389 & 1,473 & 1,531 & 1,697 & 1,811 & 1,872 & 2,066 & 2,274 & 2,490 & 2,727 \\
\hline Tax revenue & 1,238 & 1,314 & 1,354 & 1,511 & 1,511 & 1,679 & 1,855 & 2,038 & 2,239 & 2,458 \\
\hline Of which: Gold Mining CIT & 39 & 45 & 49 & 51 & 51 & 75 & 92 & 107 & 115 & 123 \\
\hline Nontax revenue & 151 & 159 & 177 & 186 & 300 & 193 & 211 & 235 & 252 & 269 \\
\hline Of which: Royalties from gold & 51 & 46 & 62 & 46 & 46 & 67 & 73 & 78 & 84 & 89 \\
\hline Grants & 194 & 322 & 215 & 240 & 258 & 244 & 250 & 265 & 280 & 297 \\
\hline Project & 120 & 174 & 122 & 159 & 164 & 167 & 167 & 176 & 184 & 193 \\
\hline Program & 74 & 147 & 93 & 80 & 93 & 77 & 82 & 89 & 96 & 104 \\
\hline Expenditure and net lending $\mathbf{1 /}$ & 2,146 & 2,166 & 2,128 & 2,196 & 2,326 & 2,395 & 2,617 & 2,864 & 3,123 & 3,405 \\
\hline Current expenditure & 1,319 & 1,365 & 1,383 & 1,597 & 1,607 & 1,649 & 1,721 & 1,858 & 1,961 & 2,124 \\
\hline Wages and salaries & 618 & 700 & 705 & 836 & 836 & 878 & 915 & 945 & 970 & 1,049 \\
\hline Goods and services & 174 & 152 & 196 & 155 & 194 & 193 & 208 & 225 & 244 & 263 \\
\hline Interest payments & 70 & 100 & 97 & 110 & 113 & 126 & 141 & 159 & 176 & 193 \\
\hline Domestic & 49 & 67 & 74 & 90 & 94 & 105 & 118 & 133 & 147 & 162 \\
\hline External & 20 & 33 & 23 & 20 & 19 & 21 & 23 & 25 & 28 & 32 \\
\hline Current transfers & 457 & 414 & 385 & 496 & 465 & 452 & 457 & 528 & 571 & 618 \\
\hline Investment expenditure & 831 & 804 & 754 & 598 & 719 & 746 & 896 & 1,007 & 1,162 & 1,281 \\
\hline Domestically financed & 587 & 495 & 529 & 308 & 424 & 454 & 604 & 690 & 819 & 909 \\
\hline Externally financed & 244 & 309 & 225 & 290 & 295 & 292 & 292 & 317 & 343 & 373 \\
\hline Net lending & -4 & -3 & -10 & 0 & 0 & 0 & 0 & 0 & 0 & 0 \\
\hline Overall balance 1/ & -563 & -371 & -382 & -259 & -258 & -279 & -301 & -326 & -352 & -381 \\
\hline Cash basis adjustment & 75 & 0 & -97 & 0 & 0 & 0 & 0 & 0 & 0 & 0 \\
\hline Overall balance (cash basis) & -488 & -371 & -478 & -259 & -258 & -279 & -301 & -326 & -352 & -381 \\
\hline Financing & 483 & 371 & 470 & 259 & 258 & 279 & 301 & 326 & 352 & 381 \\
\hline Foreign financing & 74 & 120 & 76 & 100 & 78 & 84 & 90 & 129 & 144 & 160 \\
\hline Drawings & 124 & 171 & 131 & 171 & 150 & 140 & 152 & 193 & 215 & 240 \\
\hline Project loans & 124 & 134 & 103 & 131 & 131 & 125 & 125 & 141 & 159 & 180 \\
\hline Program loans & 0 & 36 & 28 & 40 & 20 & 15 & 27 & 52 & 56 & 60 \\
\hline Amortization (excl. IMF) & -50 & -50 & -55 & -71 & -72 & -56 & -61 & -64 & -72 & -81 \\
\hline Domestic financing & 408 & 250 & 395 & 159 & 180 & 195 & 211 & 197 & 209 & 221 \\
\hline Bank financing & 108 & 250 & 142 & 159 & 180 & 195 & 211 & 197 & 209 & 221 \\
\hline Central bank & 88 & 0 & 29 & 10 & 25 & 14 & -17 & -15 & -11 & -16 \\
\hline of which: IMF net financing & -11 & 20 & 3 & 9 & 25 & 13 & -17 & -15 & -12 & -13 \\
\hline Disbursements & 4 & 28 & 22 & 28 & 43 & 29 & 0 & 0 & 0 & 0 \\
\hline Repayments & -14 & -9 & -19 & -19 & -18 & -16 & -17 & -15 & -12 & -13 \\
\hline Commercial banks & 21 & 250 & 113 & 149 & 155 & 181 & 228 & 212 & 220 & 237 \\
\hline Nonbank financing & 300 & 0 & 252 & 0 & 0 & 0 & 0 & 0 & 0 & 0 \\
\hline Errors and Omissions & 6 & & 8 & & & & & & & \\
\hline Financing gap & 0 & 0 & 0 & 0 & 0 & 0 & 0 & 0 & 0 & 0 \\
\hline
\end{tabular}

\begin{tabular}{|c|c|c|c|c|c|c|c|c|c|}
\hline 2017 & & & & & 2020 & 2021 & 2022 & 2023 & 2024 \\
\hline Act. & 1st Rev. & Prel. & 1st Rev. & Proj. & Proj. & Proj. & Proj. & Proj. & Proj. \\
\hline
\end{tabular}

Sources: Burkinabè authorities; and IMF staff estimates and projections.

1/ Commitment ("engagement") basis. 
Table 5b. Burkina Faso: Consolidated Operations of the Central Government, 2017-24 (In percent of GDP)

\begin{tabular}{|c|c|c|c|c|c|c|c|c|c|c|}
\hline & \multirow{2}{*}{$\begin{array}{l}2017 \\
\text { Act. }\end{array}$} & \multicolumn{2}{|c|}{2018} & \multicolumn{2}{|c|}{2019} & \multirow{2}{*}{$\begin{array}{l}2020 \\
\text { Proj. }\end{array}$} & \multirow{2}{*}{$\begin{array}{l}2021 \\
\text { Proj. }\end{array}$} & \multirow{2}{*}{$\begin{array}{l}2022 \\
\text { Proj. }\end{array}$} & \multirow{2}{*}{$\begin{array}{l}2023 \\
\text { Proj. }\end{array}$} & \multirow{2}{*}{$\begin{array}{l}2024 \\
\text { Proj. }\end{array}$} \\
\hline & & 1st Rev. & Prel. & 1st Rev. & Proj. & & & & & \\
\hline Total revenue and grants & 22.1 & 22.8 & 22.2 & 22.8 & 24.4 & 23.1 & 23.4 & 23.7 & 23.9 & 24.1 \\
\hline Total revenue & 19.4 & 18.7 & 19.5 & 19.9 & 21.3 & 20.4 & 20.8 & 21.2 & 21.5 & 21.8 \\
\hline Tax revenue & 17.3 & 16.7 & 17.3 & 17.8 & 17.8 & 18.3 & 18.7 & 19.0 & 19.3 & 19.6 \\
\hline Of which: Gold Mining CIT & 0.5 & 0.6 & 0.6 & 0.6 & 0.6 & 0.8 & 0.9 & 1.0 & 1.0 & 1.0 \\
\hline Nontax revenue & 2.1 & 2.0 & 2.2 & 2.2 & 3.5 & 2.1 & 2.1 & 2.2 & 2.2 & 2.1 \\
\hline Of which: Royalties from gold & 0.7 & 0.6 & 0.8 & 0.5 & 0.5 & 0.7 & 0.7 & 0.7 & 0.7 & 0.7 \\
\hline Grants & 2.7 & 4.1 & 2.7 & 2.8 & 3.0 & 2.7 & 2.5 & 2.5 & 2.4 & 2.4 \\
\hline Project & 1.7 & 2.2 & 1.6 & 1.9 & 1.9 & 1.8 & 1.7 & 1.6 & 1.6 & 1.5 \\
\hline Program & 1.0 & 1.9 & 1.2 & 0.9 & 1.1 & 0.8 & 0.8 & 0.8 & 0.8 & 0.8 \\
\hline Expenditure and net lending $\mathbf{1 /}$ & 29.9 & 27.5 & 27.1 & 25.8 & 27.4 & 26.1 & 26.4 & 26.7 & 26.9 & 27.2 \\
\hline Current expenditure & 18.4 & 17.3 & 17.6 & 18.8 & 18.9 & 18.0 & 17.4 & 17.3 & 16.9 & 16.9 \\
\hline Wages and salaries & 8.6 & 8.9 & 9.0 & 9.8 & 9.9 & 9.6 & 9.2 & 8.8 & 8.4 & 8.4 \\
\hline Goods and services & 2.4 & 1.9 & 2.5 & 1.8 & 2.3 & 2.1 & 2.1 & 2.1 & 2.1 & 2.1 \\
\hline Interest payments & 1.0 & 1.3 & 1.2 & 1.3 & 1.3 & 1.4 & 1.4 & 1.5 & 1.5 & 1.5 \\
\hline Domestic & 0.7 & 0.8 & 0.9 & 1.1 & 1.1 & 1.1 & 1.2 & 1.2 & 1.3 & 1.3 \\
\hline External & 0.3 & 0.4 & 0.3 & 0.2 & 0.2 & 0.2 & 0.2 & 0.2 & 0.2 & 0.3 \\
\hline Current transfers & 6.4 & 5.3 & 4.9 & 5.8 & 5.5 & 4.9 & 4.6 & 4.9 & 4.9 & 4.9 \\
\hline Investment expenditure & 11.6 & 10.2 & 9.6 & 7.0 & 8.5 & 8.1 & 9.0 & 9.4 & 10.0 & 10.2 \\
\hline Domestically financed & 8.2 & 6.3 & 6.7 & 3.6 & 5.0 & 5.0 & 6.1 & 6.4 & 7.1 & 7.2 \\
\hline Externally financed & 3.4 & 3.9 & 2.9 & 3.4 & 3.5 & 3.2 & 2.9 & 3.0 & 3.0 & 3.0 \\
\hline Net lending & 0.0 & 0.0 & -0.1 & 0.0 & 0.0 & 0.0 & 0.0 & 0.0 & 0.0 & 0.0 \\
\hline Overall balance 1/ & -7.8 & -4.7 & -4.9 & -3.0 & -3.0 & -3.0 & -3.0 & -3.0 & -3.0 & -3.0 \\
\hline Cash basis adjustment & 1.0 & 0.0 & -1.2 & 0.0 & 0.0 & 0.0 & 0.0 & 0.0 & 0.0 & 0.0 \\
\hline Overall balance (cash basis) & -6.8 & -4.7 & -6.1 & -3.0 & -3.0 & -3.0 & -3.0 & -3.0 & -3.0 & -3.0 \\
\hline Financing & 6.7 & 4.7 & 6.0 & 3.0 & 3.0 & 3.0 & 3.0 & 3.0 & 3.0 & 3.0 \\
\hline Foreign financing & 1.0 & 1.5 & 1.0 & 1.2 & 0.9 & 0.9 & 0.9 & 1.2 & 1.2 & 1.3 \\
\hline Drawings & 1.7 & 2.2 & 1.7 & 2.0 & 1.8 & 1.5 & 1.5 & 1.8 & 1.9 & 1.9 \\
\hline Project loans & 1.7 & 1.7 & 1.3 & 1.5 & 1.5 & 1.4 & 1.3 & 1.3 & 1.4 & 1.4 \\
\hline Program loans & 0.0 & 0.5 & 0.4 & 0.5 & 0.2 & 0.2 & 0.3 & 0.5 & 0.5 & 0.5 \\
\hline Amortization (excl. IMF) & -0.7 & -0.6 & -0.7 & -0.8 & -0.8 & -0.6 & -0.6 & -0.6 & -0.6 & -0.6 \\
\hline Domestic financing & 5.7 & 3.2 & 5.0 & 1.9 & 2.1 & 2.1 & 2.1 & 1.8 & 1.8 & 1.8 \\
\hline Bank financing & 1.5 & 3.2 & 1.8 & 1.9 & 2.1 & 2.1 & 2.1 & 1.8 & 1.8 & 1.8 \\
\hline Central bank & 1.2 & 0.0 & 0.4 & 0.1 & 0.3 & 0.1 & -0.2 & -0.1 & -0.1 & -0.1 \\
\hline of which: IMF net financing & -0.1 & 0.3 & 0.0 & 0.1 & 0.3 & 0.1 & -0.2 & -0.1 & -0.1 & -0.1 \\
\hline Disbursements & 0.1 & 0.4 & 0.3 & 0.3 & 0.5 & 0.3 & 0.0 & 0.0 & 0.0 & 0.0 \\
\hline Repayments & -0.2 & -0.1 & -0.2 & -0.2 & -0.2 & -0.2 & -0.2 & -0.1 & -0.1 & -0.1 \\
\hline Commercial banks & 0.3 & 3.2 & 1.4 & 1.8 & 1.8 & 2.0 & 2.3 & 2.0 & 1.9 & 1.9 \\
\hline Nonbank financing & 4.2 & 0.0 & 3.2 & 0.0 & 0.0 & 0.0 & 0.0 & 0.0 & 0.0 & 0.0 \\
\hline Errors and Omissions & 0.1 & & 0.1 & & & & & & & \\
\hline Financing gap & 0.0 & 0.0 & 0.0 & 0.0 & 0.0 & 0.0 & 0.0 & 0.0 & 0.0 & 0.0 \\
\hline \multicolumn{11}{|l|}{ Memorandum items: } \\
\hline Nominal GDP (CFAF billion) & 7,177 & 7,873 & 7,849 & 8,512 & 8,484 & 9,170 & 9,916 & 10,723 & 11,593 & 12,535 \\
\hline Wage bill to tax revenue ratio (percent) & 49.9 & 53.3 & 52.1 & 55.3 & 55.3 & 52.3 & 49.3 & 46.4 & 43.3 & 42.7 \\
\hline
\end{tabular}


Table 5c. Burkina Faso: Consolidated Operations of the Central Government, 2019 (CFAF billions)

\begin{tabular}{|c|c|c|c|c|c|c|c|c|c|c|c|c|}
\hline Total revenue and grants & 408 & 20 & 10 & 1,001 & 48 & 25 & 1,487 & 72 & 24 & 2,069 & 100 & 18 \\
\hline Total revenue & 396 & 22 & 19 & 934 & 52 & 27 & 1,353 & 75 & 22 & 1,811 & 100 & 18 \\
\hline Tax revenue & 336 & 22 & 12 & 737 & 49 & 13 & 1111 & 74 & 12 & 1,511 & 100 & 12 \\
\hline Nontax revenue & 60 & 20 & 83 & 197 & 66 & 139 & 242 & 81 & 114 & 300 & 100 & 70 \\
\hline Grants & 11 & 4 & -69 & 67 & 26 & -4 & 134 & 52 & 44 & 258 & 100 & 20 \\
\hline Project & 7 & 5 & -68 & 53 & 33 & 8 & 87 & 53 & 36 & 164 & 100 & 34 \\
\hline Program & 4 & 4 & -71 & 13 & 14 & -34 & 47 & 50 & 60 & 93 & 100 & 1 \\
\hline Expenditure and net lending 1/ & 399 & 17 & -15 & 1,008 & 43 & 13 & 1593 & 68 & 13 & 2,326 & 100 & 9 \\
\hline Current expenditure & 306 & 19 & -16 & 745 & 46 & 13 & 1146 & 71 & 13 & 1,607 & 100 & 16 \\
\hline Wages and salaries & 187 & 22 & 16 & 390 & 47 & 19 & 608 & 73 & 19 & 836 & 100 & 19 \\
\hline Goods and services & 22 & 11 & -53 & 82 & 42 & -4 & 125 & 64 & 6 & 194 & 100 & -1 \\
\hline Interest payments & 12 & 11 & -45 & 48 & 43 & -6 & 64 & 57 & -1 & 113 & 100 & 16 \\
\hline Domestic & 8 & 8 & -57 & 37 & 40 & -2 & 52 & 55 & 4 & 94 & 100 & 27 \\
\hline External & 4 & 23 & 19 & 11 & 57 & -18 & 13 & 69 & -19 & 19 & 100 & -19 \\
\hline Current transfers & 85 & 18 & -36 & 225 & 49 & 15 & 349 & 75 & 10 & 465 & 100 & 21 \\
\hline Investment expenditure & 93 & 13 & -16 & 264 & 37 & 9 & 448 & 62 & 10 & 719 & 100 & -5 \\
\hline Domestically financed & 64 & 15 & 13 & 154 & 36 & 12 & 277 & 65 & 4 & 424 & 100 & -20 \\
\hline Externally financed & 29 & 10 & -46 & 110 & 37 & 6 & 171 & 58 & 23 & 295 & 100 & 31 \\
\hline Net lending & -1 & & & -1 & & & & & & 0 & & \\
\hline Overall balance 1/ & 9 & & & -8 & & & -106 & & & -258 & & \\
\hline Cash basis adjustment & -92 & & & 0 & & & 0 & & & 0 & & \\
\hline Overall balance (cash basis) & -83 & & & -8 & & & -106 & & & -258 & & \\
\hline Financing & 90 & & & 8 & & & 106 & & & 258 & & \\
\hline Foreign financing & 19 & & & 33 & & & 51 & & & 78 & & \\
\hline Drawings & 30 & & & 64 & & & 91 & & & 150 & & \\
\hline Project loans & 22 & & & 56 & & & 83 & & & 131 & & \\
\hline Program loans & 8 & & & 8 & & & 8 & & & 20 & & \\
\hline Amortization (excl. IMF) & -10 & & & -31 & & & -41 & & & -72 & & \\
\hline Domestic financing & 70 & & & -26 & & & 55 & & & 180 & & \\
\hline Bank financing & 47 & & & 92 & & & 137 & & & 180 & & \\
\hline Central bank & 24 & & & 30 & & & 36 & & & 25 & & \\
\hline Commercial banks & 23 & & & 62 & & & 100 & & & 155 & & \\
\hline Nonbank financing & 24 & & & -117 & & & -81 & & & 0 & & \\
\hline Errors and Omissions & -7 & & & 0 & & & 0 & & & 0 & & \\
\hline
\end{tabular}


Table 5d. Burkina Faso: Consolidated Operations of the Central Government, 2020

(CFAF billions)

\begin{tabular}{|c|c|c|c|c|c|c|c|c|c|c|c|c|}
\hline \multirow[b]{3}{*}{ Total revenue and grants } & \multicolumn{3}{|c|}{ March } & \multicolumn{3}{|c|}{ June } & \multicolumn{3}{|c|}{ September } & \multicolumn{3}{|c|}{ December } \\
\hline & Prog. & $\begin{array}{l}\text { Exec. Rate } \\
\text { (pct.) }\end{array}$ & $\begin{array}{l}Y-o-Y(\text { pct. } \\
\text { ch.) }\end{array}$ & Prog. & $\begin{array}{l}\text { Exec. Rate } \\
\text { (pct.) }\end{array}$ & $\begin{array}{c}Y-o-Y(p c t . \\
\text { ch.) }\end{array}$ & Prog. & $\begin{array}{l}\text { Exec. Rate } \\
\text { (pct.) }\end{array}$ & $\begin{array}{l}Y-o-Y \text { (pct. } \\
\text { ch.) }\end{array}$ & Prog. & $\begin{array}{l}\text { Exec. Rate } \\
\text { (pct.) }\end{array}$ & $\begin{array}{l}Y-o-Y \text { (pct. } \\
\text { ch.) }\end{array}$ \\
\hline & 455 & 21 & 12 & 1,016 & 48 & 1 & 1,542 & 73 & 4 & 2,116 & 100 & 2 \\
\hline Total revenue & 414 & 22 & 5 & 921 & 49 & -1 & 1,385 & 74 & 2 & 1,872 & 100 & 3 \\
\hline Tax revenue & 382 & 23 & 14 & 828 & 49 & 12 & 1243 & 74 & 12 & 1,679 & 100 & 11 \\
\hline Nontax revenue & 33 & 17 & -46 & 93 & 48 & -53 & 142 & 73 & -41 & 193 & 100 & -36 \\
\hline Grants & 40 & 17 & 255 & 95 & 39 & 42 & 157 & 64 & 17 & 244 & 100 & -5 \\
\hline Project & 32 & 19 & 329 & 79 & 47 & 47 & 113 & 68 & 30 & 167 & 100 & 2 \\
\hline Program & 8 & 11 & 115 & 16 & 21 & 21 & 44 & 57 & -7 & 77 & 100 & -18 \\
\hline Expenditure and net lending 1/ & 452 & 19 & 13 & 1,114 & 47 & 11 & 1717 & 72 & 8 & 2,395 & 100 & 3 \\
\hline Current expenditure & 346 & 21 & 13 & 794 & 48 & 7 & 1205 & 73 & 5 & 1,649 & 100 & 3 \\
\hline Wages and salaries & 205 & 23 & 10 & 418 & 48 & 7 & 647 & 74 & 6 & 878 & 100 & 5 \\
\hline Goods and services & 35 & 18 & 59 & 94 & 49 & 16 & 137 & 71 & 10 & 193 & 100 & -1 \\
\hline Interest payments & 12 & 10 & 2 & 52 & 42 & 9 & 71 & 56 & 10 & 126 & 100 & 12 \\
\hline Domestic & 9 & 9 & 19 & 42 & 40 & 13 & 58 & 56 & 13 & 105 & 100 & 12 \\
\hline External & 3 & 15 & -29 & 10 & 48 & -5 & 13 & 60 & -2 & 21 & 100 & 12 \\
\hline Current transfers & 93 & 21 & 10 & 230 & 51 & 2 & 350 & 77 & 0 & 452 & 100 & -3 \\
\hline Investment expenditure & 106 & 14 & 13 & 320 & 43 & 21 & 512 & 69 & 14 & 746 & 100 & 4 \\
\hline Domestically financed & 46 & 10 & -29 & 180 & 40 & 17 & 311 & 69 & 13 & 454 & 100 & 7 \\
\hline Externally financed & 60 & 21 & 106 & 140 & 48 & 27 & 201 & 69 & 17 & 292 & 100 & -1 \\
\hline Net lending & 0 & & & 0 & & & & & & 0 & & \\
\hline Overall balance 1/ & 3 & & & -99 & & & -175 & & & -279 & & \\
\hline Cash basis adjustment & 0 & & & 0 & & & 0 & & & 0 & & \\
\hline Overall balance (cash basis) & 3 & & & -99 & & & -175 & & & -279 & & \\
\hline Financing & -3 & & & 99 & & & 175 & & & 279 & & \\
\hline Foreign financing & 20 & & & 36 & & & 55 & & & 84 & & \\
\hline Drawings & 28 & & & 61 & & & 87 & & & 140 & & \\
\hline Project loans & 28 & & & 61 & & & 87 & & & 125 & & \\
\hline Program loans & 0 & & & 0 & & & 0 & & & 15 & & \\
\hline Amortization (excl. IMF) & -8 & & & -24 & & & -32 & & & -56 & & \\
\hline Domestic financing & -23 & & & 62 & & & 120 & & & 195 & & \\
\hline Bank financing & 49 & & & 98 & & & 146 & & & 195 & & \\
\hline Central bank & 3 & & & 7 & & & 10 & & & 14 & & \\
\hline Commercial banks & 45 & & & 91 & & & 136 & & & 181 & & \\
\hline Nonbank financing & -72 & & & -35 & & & -26 & & & 0 & & \\
\hline Errors and Omissions & 0 & & & 0 & & & 0 & & & 0 & & \\
\hline
\end{tabular}


Table 6. Burkina Faso: External Financing Requirements, 2017-21

(CFAF billions)

Financing need

Current account balance (excl. official transfers)

IMF repayments

Private capital flows

Amortization of public loans (excl. IMF)

Financing

Project loans:

of which: World Bank

AfDB

Islamic Development Bank

BOAD

Program loans (excl. IMF):

of which: World Bank

AfDB

Official transfers, net

of which: budget support EU

World Bank

Denmark

Swizterland

France

NFA central bank (excl. prospective IMF

disbursements and repayments)

Project grants

Eurobond

Errors and Omissions

Residual Gap

IMF ECF-Financing (past and prospective)

\begin{tabular}{|c|c|c|c|c|}
\hline 2017 & 2018 & 2019 & 2020 & 2021 \\
\hline Out. & Prel. & Proj. & Proj. & Proj. \\
\hline-229 & -76 & -394 & -343 & -325 \\
\hline-620 & -564 & -615 & -566 & -557 \\
\hline-14 & -19 & -18 & -16 & -17 \\
\hline 456 & 562 & 311 & 295 & 310 \\
\hline-50 & -55 & -72 & -56 & -61 \\
\hline 230 & 57 & 351 & 314 & 325 \\
\hline 124 & 103 & 131 & 125 & 125 \\
\hline 40 & 20 & & & \\
\hline 14 & 20 & & & \\
\hline 19 & 24 & & & \\
\hline 13 & 14 & & & \\
\hline 0 & 28 & 20 & 15 & 27 \\
\hline 0 & 28 & 16 & & \\
\hline 0 & 0 & 4 & & \\
\hline 100 & 110 & 97 & 80 & 86 \\
\hline 52 & 58 & 68 & & \\
\hline 0 & 14 & 13 & & \\
\hline 11 & 9 & 3 & & \\
\hline 4 & 4 & 4 & & \\
\hline 7 & 7 & 4 & & \\
\hline-115 & -307 & -61 & -73 & -80 \\
\hline 120 & 122 & 164 & 167 & 167 \\
\hline 0 & 0 & 0 & 0 & 0 \\
\hline-4 & 5 & 0 & 0 & 0 \\
\hline-4 & -14 & -43 & -29 & 0 \\
\hline 4 & 14 & 43 & 29 & 0 \\
\hline
\end{tabular}


Table 7. Burkina Faso: Schedule of Disbursements Under Three-year ECF Arrangement, 2018-20

\section{Amount}

SDR 18.06 million

SDR 18.06 million

SDR 18.06 million

SDR 18.06 million

SDR 18.06 million

SDR 18.06 million
Availability date

March 14, 2018

December 15, 2018

June 15, 2019

December 15, 2019

June 15, 2020

December 15, 2020

\section{Conditions for disbursement $\mathbf{1 /}$}

Executive Board Approval of a new arrangement under the Extended Credit Facility

Observance of continuous and end-June 2018 performance criteria, and completion of the first review under the arrangement

Observance of continuous and end-December 2018 performance criteria, and completion of the second review under the arrangement

Observance of continuous and end-June 2019 performance criteria, and completion of the third review under the arrangement

Observance of continuous and end-December 2019 performance criteria, and completion of the fourth review under the arrangement

Observance of continuous and end-June 2020 performance criteria and completion of the fifth review under the arrangement

Sources: Burkinabè authorities; and IMF staff estimates.

1/ In addition to the generally applicable conditions under the Extended Credit Facility. 


\begin{tabular}{|c|c|c|c|c|c|c|c|c|c|c|c|c|c|c|c|c|c|}
\hline & 2017 & 2018 & 2019 & 2020 & 2021 & 2022 & 2023 & 2024 & 2025 & 2026 & 2027 & 2028 & 2029 & 2030 & 2031 & 2032 & 2033 \\
\hline & Act. & Prel. & & & & & & & & ojections & & & & & & & \\
\hline \multicolumn{18}{|c|}{$\begin{array}{l}\text { Fund obligations based on existing and prospective credit } \\
\text { (in millions of SDRs) }\end{array}$} \\
\hline Principal & 17.8 & 24.1 & 22.7 & 19.0 & 21.2 & 18.3 & 14.2 & 16.3 & 15.0 & 10.9 & 9.9 & 3.6 & 1.8 & 0.0 & 0.0 & 0.0 & 0.0 \\
\hline Charges and interest & 0.0 & 0.0 & 0.2 & 0.4 & 0.4 & 0.4 & 0.4 & 0.4 & 0.4 & 0.4 & 0.4 & 0.4 & 0.4 & 0.4 & 0.4 & 0.4 & 0.4 \\
\hline \multicolumn{18}{|c|}{ Total obligations based on existing and prospective credit } \\
\hline In millions of SDRs & 17.8 & 24.1 & 22.9 & 19.5 & 21.6 & 18.7 & 14.6 & 16.7 & 15.4 & 11.3 & 10.3 & 4.0 & 2.2 & 0.4 & 0.4 & 0.4 & 0.4 \\
\hline In billions of CFAF & 14.3 & 19.0 & 18.3 & 15.5 & 17.2 & 14.9 & 11.7 & 13.4 & 12.4 & 9.0 & 8.3 & 3.2 & 1.8 & 0.3 & 0.3 & 0.3 & 0.3 \\
\hline In percent of government revenues & 1.0 & 1.2 & 1.0 & 0.8 & 0.8 & 0.7 & 0.5 & 0.5 & 0.4 & 0.3 & 0.2 & 0.1 & 0.0 & 0.0 & 0.0 & 0.0 & 0.0 \\
\hline In percent of exports of goods and services & 0.7 & 0.8 & 0.7 & 0.6 & 0.6 & 0.5 & 0.3 & 0.4 & 0.3 & 0.2 & 0.2 & 0.1 & 0.0 & 0.0 & 0.0 & 0.0 & 0.0 \\
\hline In percent of debt service $2 /$ & 18.1 & 18.3 & 16.8 & 16.8 & 17.0 & 14.4 & 10.5 & 10.4 & 7.9 & 5.0 & 4.3 & 1.6 & 0.8 & 0.1 & 0.1 & 0.1 & 0.1 \\
\hline In percent of GDP & 0.2 & 0.2 & 0.2 & 0.2 & 0.2 & 0.1 & 0.1 & 0.1 & 0.1 & 0.1 & 0.1 & 0.0 & 0.0 & 0.0 & 0.0 & 0.0 & 0.0 \\
\hline In percent of quota & 14.8 & 20.1 & 19.0 & 16.2 & 17.9 & 15.5 & 12.2 & 13.9 & 12.8 & 9.4 & 8.6 & 3.3 & 1.8 & 0.3 & 0.3 & 0.3 & 0.3 \\
\hline \multicolumn{18}{|l|}{ Outstanding IMF credit } \\
\hline In millions of SDRs & 141.9 & 135.8 & 166.3 & 183.4 & 162.3 & 144.0 & 129.8 & 113.5 & 89.4 & 64.1 & 39.7 & 21.7 & 5.4 & 0.0 & 0.0 & 0.0 & 0.0 \\
\hline In billions of CFAF & 114.3 & 106.7 & 133.3 & 146.5 & 129.6 & 115.1 & 103.8 & 90.8 & 71.6 & 51.3 & 31.8 & 17.3 & 4.3 & 0.0 & 0.0 & 0.0 & 0.0 \\
\hline In percent of government revenues & 8.2 & 7.0 & 7.4 & 7.8 & 6.3 & 5.1 & 4.2 & 3.3 & 2.4 & 1.6 & 0.9 & 0.4 & 0.1 & 0.0 & 0.0 & 0.0 & 0.0 \\
\hline In percent of exports of goods and services & 5.3 & 4.4 & 5.2 & 5.3 & 4.4 & 3.7 & 3.1 & 2.5 & 1.9 & 1.3 & 0.8 & 0.4 & 0.1 & 0.0 & 0.0 & 0.0 & 0.0 \\
\hline In percent of debt service $2 /$ & 144.0 & 103.0 & 122.3 & 158.6 & 127.9 & 110.8 & 93.2 & 70.9 & 45.8 & 28.7 & 16.5 & 8.6 & 2.0 & 0.0 & 0.0 & 0.0 & 0.0 \\
\hline In percent of GDP & 1.6 & 1.4 & 1.6 & 1.6 & 1.3 & 1.1 & 0.9 & 0.7 & 0.5 & 0.4 & 0.2 & 0.1 & 0.0 & 0.0 & 0.0 & 0.0 & 0.0 \\
\hline In percent of quota & 117.8 & 112.8 & 138.2 & 152.4 & 134.8 & 119.6 & 107.8 & 94.3 & 74.3 & 53.2 & 33.0 & 18.0 & 4.5 & 0.0 & 0.0 & 0.0 & 0.0 \\
\hline Net use of IMF credit (in millions of SDRs) & -13.3 & -6.1 & 31.3 & 16.7 & -21.6 & -18.7 & -14.6 & -16.7 & -15.4 & -11.3 & -10.3 & -4.0 & -2.2 & -0.4 & -0.4 & -0.4 & -0.4 \\
\hline Disbursements & 4.5 & 18.1 & 54.2 & 36.1 & 0.0 & 0.0 & 0.0 & 0.0 & 0.0 & 0.0 & 0.0 & 0.0 & 0.0 & 0.0 & 0.0 & 0.0 & 0.0 \\
\hline Repayments and repurchases & 17.8 & 24.1 & 22.9 & 19.5 & 21.6 & 18.7 & 14.6 & 16.7 & 15.4 & 11.3 & 10.3 & 4.0 & 2.2 & 0.4 & 0.4 & 0.4 & 0.4 \\
\hline \multicolumn{18}{|l|}{ Memorandum items: } \\
\hline Nominal GDP (in billions of CFAF) & 7,177 & 7,849 & 8,484 & 9,170 & 9,916 & 10,723 & 11,593 & 12,535 & 13,552 & 14,653 & 15,843 & 17,129 & 18,520 & 20,024 & 21,650 & 23,408 & 25,309 \\
\hline Exports of goods and services (in billions of CFAF) & 2,167 & 2,408 & 2,542 & 2,750 & 2,961 & 3,147 & 3,350 & 3,565 & 3,747 & 3,941 & 4,146 & 4,363 & 4,586 & 4,821 & 5,069 & 5,332 & 5,609 \\
\hline Government revenue (in billions of CFAF) & 1,389 & 1,531 & 1,811 & 1,872 & 2,066 & 2,274 & 2,490 & 2,727 & 2,981 & 3,276 & 3,580 & 3,877 & 4,200 & 4,550 & 4,907 & 5,293 & 5,710 \\
\hline Debt service (in billions of CFAF) 2/3/ & 79 & 104 & 109 & 92 & 101 & 104 & 111 & 128 & 156 & 179 & 193 & 201 & 219 & 224 & 239 & 269 & 302 \\
\hline CFAF/SDR (period average) & 805 & 786 & 801 & 799 & 799 & 799 & 800 & 800 & 800 & 800 & 800 & 800 & 800 & 800 & 800 & 800 & 800 \\
\hline \multicolumn{18}{|c|}{$\begin{array}{l}\text { Sources: IMF staff estimates and projections. } \\
\text { 1/ Includes proposed disbursements under the new ECF of } 90 \text { percent of quota. } \\
2 / \text { Total external debt service includes IMF repurchases and repayments. } \\
\text { 3/ Includes state-owned enterprises debt. }\end{array}$} \\
\hline
\end{tabular}




\section{Annex I. Risk Assessment Matrix ${ }^{1}$}

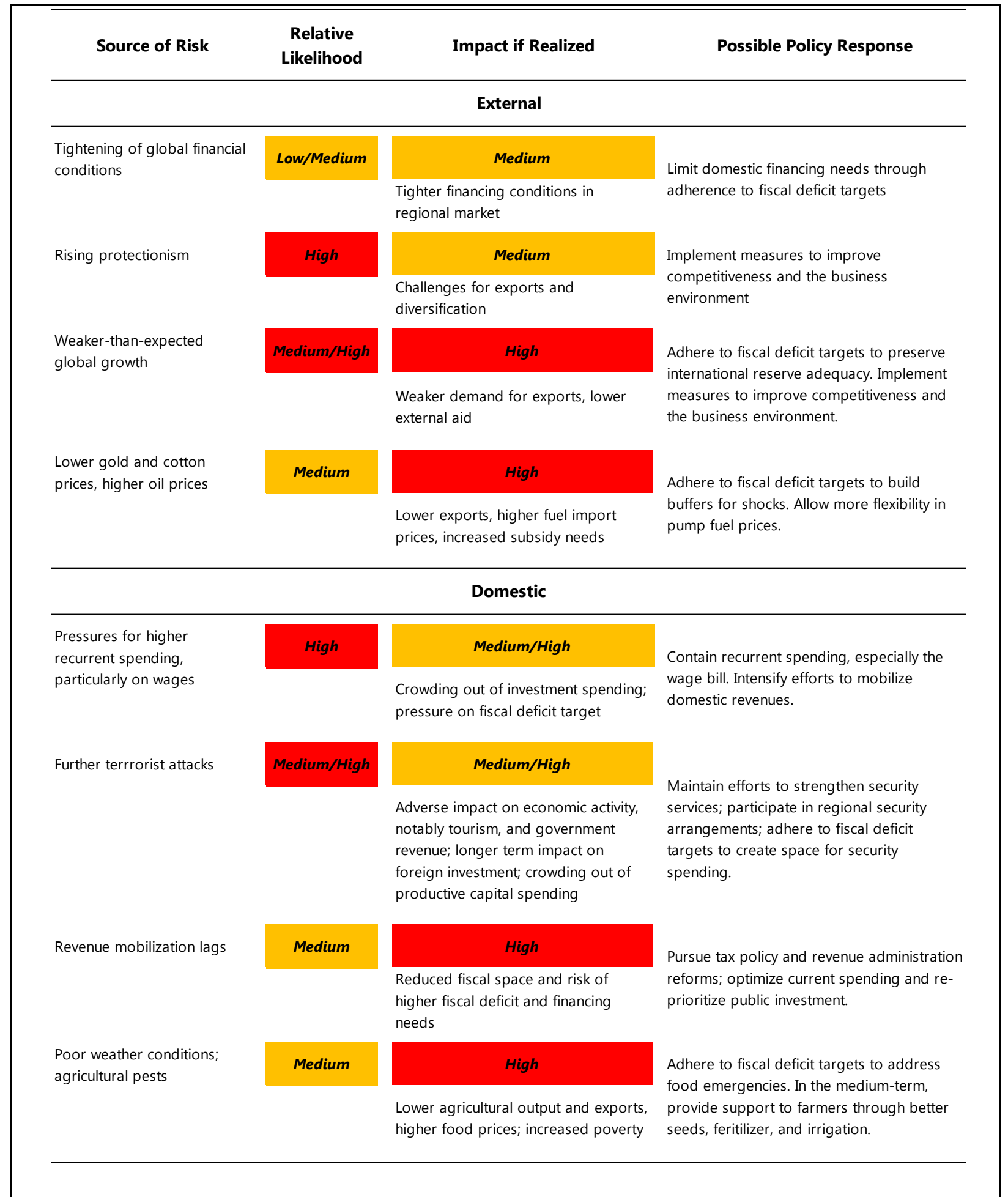

${ }^{1}$ The Risk Assessment Matrix (RAM) shows events that could materially alter the baseline path (the scenario most likely to materialize in the view of IMF staff. The likelihood of risks listed is the staff's subjective assessment of the risks surrounding the baseline. The RAM reflects staff views on the source of risks and overall level of concern as of the time of discussions with the authorities. Non-mutually exclusive risks may materialize jointly and interact. 
BURKINA FASO

MINISTRY OF ECONOMY, FINANCE

AND DEVELOPMENT

Ouagadougou, June 27, 2019

\title{
SECRETARIAT GENERAL
}

\section{DIRECTORATE GENERAL}

FOR COOPERATION

No. 2019____MINEFID/SG/DGCOOP

The Ministry of Economy, Development and Finance

\author{
To \\ Madame Christine Lagarde \\ Managing Director \\ International Monetary Fund \\ 700 19th Street NW \\ Washington, DC 20431 (USA)
}

SUBJECT: Letter of intent on economic and financial policies

\section{Madame Managing Director,}

In 2018, Burkina Faso's 2018-2020 economic and financial program supported by the Extended Credit Facility (ECF) was implemented amid a difficult security situation and continued labor strikes. Despite that context, the Burkinabè economy posted solid performance in 2018, with real GDP growth of 6.8 percent and inflation remaining contained at an annual average of 2 percent, below the WAEMU regional norm of 3 percent. Growth is projected to slow in 2019, to 6 percent, and inflation to remain below the WAEMU regional norm.

Performance under the program was broadly satisfactory. All the quantitative performance criteria and indicative objectives were met except for the performance criterion relating to the ceiling on net domestic financing of the government at end-2018 and end-March 2019. The financing in excess of the ceiling is explained by the need to pay down in 2018 the committed expenditures without 
payment orders (DENO) accumulated in 2017 and to pay current-year expenditures. This led to increased cash flow requirements in 2018; hence the increased issuance of government securities.

To avoid a situation of this kind in the future, enhanced monitoring of investment programming has been put in place. Improved planning and spreading of commitments over the entire year should also help avoid the excessive accumulation of DENO. In addition, we will evaluate the adequacy of our expenditure monitoring and execution mechanisms with the IMF resident advisor during his visit in July 2019.

\section{While assuring you of its firm commitment to continue and strengthen its partnership with the IMF, the government requests a waiver for nonobservance of the performance criterion relating to the ceiling on net domestic financing of the government at end-December 2018.}

The majority of the program's structural benchmarks have been met, with substantial progress made on the ones that have not been met and the ones whose test dates have not yet passed.

In working to achieve the program objectives, the government will continue its efforts to consolidate public finances and implement the reforms needed to increase revenue and improve expenditure monitoring. Accordingly, the reform measures aimed at increasing revenue and management of expenditure will be continued and intensified.

The overall deficit is expected to stand at 3.0 percent of GDP in 2019, a decline of 1.9 percentage points with respect to 2018. The deficit will remain at this level in 2020, consistent with our commitments under our ECF-supported program and with WAEMU convergence criteria. It will be financed in 2019 and 2020 through the use of budgetary support, drawings on IMF resources, and the issuance of securities on the financial and money markets. Windfall revenues pertaining to $4 G$ telecommunication licenses will be allocated in the supplementary budget of 2019 essentially to investments and in part to security spending.

Concerning the management of the wage bill, in view of reducing the wage bill as a percentage of tax revenue in the medium term to the level required by WAEMU directives, the government will continue the reforms begun in 2018 and earlier. The government is committed in particular to preparing a medium-term strategy document on civil service compensation (covering the organic law, and the salary indexation and indemnity scales) in the context of implementing the conclusions of the national conference on rationalizing the civil service compensation system. A communications campaign on the wage bill strategy is conducted at the highest levels of government to inform populations of the consequences of disproportionate growth of the wage bill. This strategy includes two actions undertaken in June 2019: a public announcement by the government on its commitment to gradually reduce the wage bill as a proportion of tax revenue starting in 2020, and the publication of the number and repartition of public servants according to grade, region and ministry. Moreover, the government plans to organize a conference and present the results of the work on the civil service compensation system in the very near future. Pending the implementation of the totality of its reform package, the government commits to contain the public wage bill in 2019 and 2020 within the levels envisaged during the first program review and to abstain from concluding any new agreement that might induce an increase of the public wage bill. 
Concerning the fuel price adjustment mechanism implemented in 2018, the authorities agree to continue efforts to improve the mechanism's functioning. The government plans to continue a course of action designed to ensure the viability of national oil company SONABHY (Société nationale burkinabé d'hydrocarbures), safeguard its mission of supplying the country, and reduce fuel subsidies in order to free up resources to support investment and the social sectors. To this end, the implementation of the mechanism will be delegated to a technical unit that will automatically communicate the new real prices to the public at the beginning of each quarter. The government retains the prerogative to define the policy for fuel price adjustment and a level of discretion in its readjustment.

In view of its commitment to preserve macroeconomic stability, and confident in the policies set out in the accompanying memorandum of economic and financial policies (MEFP), the government requests that the IMF conclude the second review under the ECF arrangement. To that end, the government requests the disbursement of SDR 18.06 million, or CFAF 14.27 billion.

The government is confident that the measures set forth in the MEFP will serve to achieve the economic and social objectives of its program. Nevertheless, it stands ready to take any further measures that may prove necessary to that end.

The government will consult the IMF prior to the adoption of such measures, and prior to any revision of the policies set out in the MEFP, in accordance with Fund policies on such consultations. It will provide the IMF with any information relating to the implementation of agreed measures and program implementation, as provided in the accompanying revised Technical Memorandum of Understanding or at IMF's request.

As in the past, the government authorizes the IMF to disclose this letter and attachments and the IMF staff report once they are approved by the IMF Executive Board.

Very truly yours,

/s/

\section{Lassané Kabore}

Minister of Economy, Finance and Development

Chevalier de I'Ordre National

\section{Attachments:}

- Memorandum on Economic and Financial Policies

- Technical Memorandum of Understanding 


\section{Attachment 1. Memorandum of Economic and Financial Policies}

\section{INTRODUCTION}

1. This Memorandum of Economic and Financial Policies (MEFP) updates data on the implementation of Burkina Faso's Economic and Financial Program with the International Monetary Fund (IMF) under the Extended Credit Facility for 2018 -2020.

2. It describes the recent trends in Burkina Faso's economy and reports on performance under the program supported by the Extended Credit Facility Arrangement at end December 2018 and end March 2019. It also presents the main guidelines for the rest of 2019.

3. The objectives of the program are mainly geared towards maintaining macroeconomic stability while creating fiscal space through increased domestic revenue mobilization and improved public spending. This program is in line with the regional commitments made by our country, particularly that of reducing the fiscal deficit to a compatible level while preserving critical spending on social services and priority public investments.

\section{RECENT ECONOMIC DEVELOPMENTS AND PROGRAM IMPLEMENTATION}

4. The national economy performed well in 2018 despite a challenging security situation and continued social demands. As part of implementation of the program, at December 2018 and end March 2019, all quantitative performance criteria and indicative targets were met except for the performance criterion relating to net domestic financing of the State. Most of the structural benchmarks have been met.

\section{A. Recent Economic Developments at End-2018 and End-March 2019}

5. Based on our latest estimates, the real GDP growth rate stood at $6.8 \%$ in 2018 compared with $6.3 \%$ in 2017 . This growth acceleration is mainly driven by the primary sector $(9.9 \%)$, while the secondary and tertiary sectors grew by $3.6 \%$ and $6.7 \%$ respectively. At end December 2018 , the inflation rate averaged $2 \%$ compared with $0.4 \%$ in the previous year. Inflation was controlled thanks to the good performance of the 2018/2019 agricultural season and the continuation of government measures to contain high cost of living (sale of cereals at social prices in deficit areas, control of consumer goods prices, opening subsidized grain sales points). At end March 2019, the inflation rate was 1\% as against 1.7\% in March 2018.

6. Foreign trade was characterized in 2018 by higher export growth than imports, in a context marked by a contrasting trend in the main export products (rise in the price of cotton fibre and that of gold, a drop in cotton production and a rise in gold production) and the rise in the oil bill. In addition, there was an estimated slight improvement in net capital inflows as part of the 
mobilization of external financing in support of the National Economic and Social Development Plan (PNDES) and a reduction in net financial flows related to repayments of contracted debts, particularly for mining investments. Overall, transactions with the rest of the world is believed to have resulted in a reduction of the current account deficit and a surplus of the overall balance of payments in 2018, after a higher surplus in 2017. Thus, the overall balance of the balance of payments was estimated at CFAF 113.4 billion in 2018, after a CFAF 247 billion surplus in 2017, a deterioration of CFA F133.6 billion over one year.

7. The monetary situation at end December 2018 reflects a $10.3 \%$ increase in the money supply compared with end December 2017, in connection with the $11.8 \%$ increase in domestic receivables and an $8.5 \%$ increase in net foreign assets (NFA) from deposit institutions. The rise in domestic receivables is attributable exclusively to a $12.4 \%$ increase in claims on the economy, as a result of the increase in bank credit to the private sector, mitigated by the $10.2 \%$ decline in net claims on the Central Administration. At end December 2018, loans to the economy accounted for 33.9\% of GDP compared with 33.2\% at end December 2017. At end March 2019, money supply grew by $4.8 \%$ compared with end December 2018, under the impact of the increase in NFA (+4.8\%) and domestic debt $(+3.1 \%)$. During this quarter, credit to the economy grew by $2 \%$, compared with an increase of $4.3 \%$ in the first quarter of 2018 year-on-year. NFA growth is attributable to those of the Central Bank of West Africa States (BCEAO) (CFAF +311.5 billion) and was mitigated by the decrease in the NFA of the Other Deposit-taking Institutions (AID) (CFAF -198.1 billion). The Central Bank's NFA increase is linked to a decline in its gross commitments to non-residents (CFAF -279.7 billion), boosted by the increase in its gross claims on non-residents (CFAF +31.8 billion). The decline of the commercial banks' NFA (-14.9\%) is linked in particular to the decline in their outstanding public securities in other Union States (-15.8\%), the decline in outstanding loans granted to non-residents $(-24.5 \%)$ and the decline in deposits made by local banks with non-resident financial institutions (16.2\%). At the same time, there was a decrease in their foreign liabilities (CFAF -173.3 billion), driven by the decrease in credits received from non-resident financial institutions (-37.2\%) and deposits by non-residents (- 20.4\%).

8. In terms of public finances, total revenue and grants mobilized at end December 2018 stood at CFAF 1,745.95 billion against CFAF 1,583.57 billion in the same period in 2017, being a CFAF 162.38 billion increase (+10.25\%). They accounted for $22.4 \%$ of GDP at end December 2018 compared with $22.06 \%$ of GDP during the same period in 2017. This increase is due to the increase of the own revenues worth CFAF 141,59 billion and that of grants worth CFAF 20,79 billion (+10,19\%). At end March 2019, total income and grants raised amounted to CFAF 416.61 billion against CFAF 370.94 billion in the same period in 2018, an increase of $12.31 \%$.

9. Own revenue amounted to CFAF $1,530.86$ billion at end December 2018, up by $10.19 \%$ compared with end December 2017. Tax revenue amounting to CFAF 1354.27 billion increased by 9.37\%, that is CFAF 116.02 billion, compared with end December 2017. Non-tax revenue increased by CFAF 25.57 billion (+16.93\%) at end December 2018 compared with the same period in 2017. The tax burden rate stabilized at 17.25\% of GDP in 2018 just like in 2017. At end March 2019, mobilized 
tax revenues amounted to CFAF 333.01 billion against CFAF 301.15 billion in the same period in 2018 , an increase of $10.58 \%$.

10. Mining revenue collected at end December 2018 stood at CFAF 253.40 billion (including CFAF 187.77 billion of tax revenue) against CFAF 223.45 billion (including CFAF 164.65 billion of tax revenue) at 31 December 2017, an increase of CFAF 29.95 billion (+13.40\%). They are expected to account for $3.23 \%$ of GDP in 2018 against $3.11 \%$ in 2017 . Such performance could be explained by the increase in gold production combined with the favourable price trend of the ounce of gold. At end March 2019, mining revenues reached CFAF 63.13 billion against CFAF 51.12 billion in the same period in 2018 , an increase of $23.50 \%$.

11. Despite a difficult social and security context, the performances achieved in the mobilization of own resources are mainly attributable to the following measures and actions:

- operationalization by the Directorate General of Taxes (DGI) of tax procedures allowing online tax declarations and payments, intensive recovery of outstanding receivables and visits to companies by heads of taxation services to support and facilitate the collection of taxes;

- intensification of investigation and cross-checking with a view to improving tax auditing through spot checks;

- reinforced control of service execution in offices by the Regional Directors and audits of the Technical Inspectorate;

- strengthening of the skills of auditors and the staff for a better evaluation of goods according to the WTO value within the framework of the exit of the Pre-Shipment Inspection Program through the fight against all forms of customs fraud (contraband, undeclared importation, misrepresentation and non-fulfilment of commitments made);

- strengthening control of the mining sector.

12. Grants mobilization amounted to CFAF 215.09 billion at end December 2018 against CFAF 194.30 billion at end December 2017, an increase of CFAF 20.79 billion (+10.70\%). This trend is attributable to the increase in program grants of CFAF 18.34 billion and CFAF 2.44 billion in project grants. At end March 2019, there was a contraction in the level of grants, which amounted to CFAF 11.36 billion against CFAF 36.97 billion in the same period in 2018.

13. Total expenditure and net loans were executed to the tune of CFAF 2,127.61 billion at end December 2018 against CFAF 2,146.47 billion for the same period in 2017, representing a contraction of CFAF 18.86 billion (-0.88\%). These expenditure execution levels account respectively for $27.11 \%$ of GDP at end December 2018 compared with $29.91 \%$ at the same period of 2017 . This drop is a result of a CFAF 76.99 billion decrease in capital expenditure and a current expenditure increase of CFAF 64.70 billion.

14. The decline in capital expenditure to CFAF 754.34 billion (9.61\% of GDP) at end December 2018 can be explained notably by the social unrests affecting the expenditure processing chain and the shrinking of resources for investments. 
15. The increase in current expenditure (4.91\%) compared with end December 2017) is mainly attributable to the increase in personnel expenditure of $14.10 \%$ (+CFAF 87.16 billion) and a $39.88 \%$ interest on the debt (+CFAF 27.73 billion). The increase in personnel expenditure is explained by the implementation of the commitments (memoranda of understanding) concluded with social partners, the additional staff and statutory advancements. The increase in interest on debt is due to those paid in respect of domestic debt, linked to the volume of issues in a context of rising interest rates.

16. At end March 2019, total expenditures and net loans were executed at CFAF 399.11 billion against CFAF 470.12 billion in the same period in 2018. This low spending performance in the first quarter of 2019 compared with the first quarter of 2018 is due to social unrest and a desire to streamline current expenses. In fact, current expenditure was executed at CFAF 306.42 billion against CFAF 364.43 billion in the same period in 2018, a decrease of $15.92 \%$. Investment expenses were executed to the tune of 93.20 billion against 110.68 billion, registering a $15.80 \%$ decline.

17. Due to the increasingly growing share of current expenditure in total expenditures and own resources, particularly the wage component, Government has taken measures to contain them. These measures concern improved staff strength management and the reform of the remuneration system of government employees.

18. As part of improving staff strength management, the Government (i) effected a reduction of $41,8 \%$ ( 6326 positions actually filled) and $44.5 \%$ ( 5892 selected positions to be filled) in the number of new staff to be recruited in 2018 and 2019 compared with the initial projections of 10,868 and 10,615 respectively, and (ii) adopted a decree relating to the description of workstations in the public administration to ensure a better match between staff and needs (workstations are described for 14 (fourteen) ministries, while the process is ongoing for the remaining ministries). In addition, to improve the management of jobs and make realistic recruitment in accordance with the instruments, the Government has begun preparing a draft decree on the inter-ministerial directory of State trades (RIME) which is currently being finalized. This will be followed by the finalization and adoption of the various special rules and regulations signed in implementation of the general rules and regulations (these are decrees organizing jobs: responsibilities, methods and conditions of access, classifications by category, transitional provisions). Still in view of containing the staff strength, an administrative census of government employees has been carried out and the results are being finalized.

19. Regarding the reform of the remuneration system of government employees, a monitoring committee and a technical secretariat, responsible for implementing the recommendations of the national Conference of the live forces of the Nation on the remuneration system of government employees held in June 2018, were set up and are functional. As part of implementation of the conclusions of this Conference, several actions have been initiated:

- preparation of a preliminary draft organic law to lay down the fundamental principles and values of the civil service applicable to all State employees. It remains to be adopted by the Council of Ministers and then tabled before Parliament. However, there is 
currently a debate about its constitutional basis at the level of the political authorities that are trying to respond thereto;

- preparation of harmonized remuneration instruments (index and allowance scales) in implementation of the draft organic law. The purpose of these remuneration instruments is to provide a sustainable mechanism for a single remuneration mechanism for State employees based on the dual principle of equity between State employees and budget sustainability;

- a draft decree on the remuneration of senior State officials is being finalized. The aim is to have an objective and transparent basis for fixing their remuneration with a view to rationalization;

- the review of the decree on the standard organization of ministerial departments is in progress. The objective of this review is to ensure consistency between the central and decentralized administrative structures and the program budget, all of which will make it possible to reduce the number of structures and administrative officials and hence the related charges.

20. The instruments drafted in view of implementation of recommendations of the Conference of the live forces of the Nation on streamlining the remuneration system of government employees should be subject to a "review conference" that will help make the necessary amendments to those instruments, but especially to provide guidance towards accelerating their finalization.

21. The overall commitment-basis deficit stood at CFAF 381.65 billion in 2018 against a deficit of CFAF 562.90 billion a year earlier. It accounted for 4.86\% of GDP in 2018 against $7.84 \%$ in 2017.

22. As part of improving the quality of capital expenditure, the Government has adopted a new general regulation for development projects and programs, namely Decree No. 2018-

0092/PRES/PM/MINEFID of 15 February 2018. New criteria have been introduced in the selection of projects and programs. It consists in selecting only new projects that have been evaluated ex-ante and have an investment share of at least $85 \%$ for category 1 projects (projects implemented by the administration) and $75 \%$ for category 2 projects (projects carried out by an executing agency). In addition, in February 2019, the Government adopted Decree No. 2019-0141/PRES/PM/MINIFID: national guide for maturing and ex-ante evaluation of development projects and programs. The guide provides stakeholders with a harmonized approach to the process of developing and maturing public investment projects and programs. In addition, under the new regulations, draft instruments on the creation and management of development projects and programs have been developed to guide ministries and institutions in this area. The development projects and programs monitoring and evaluation module has been included in the External Finance Integrated Circuits (EFIC) platform. Accordingly, trainings have been organized for the actors involved in the design, implementation and monitoring-evaluation of projects, with a view to building their capacities to use the EFIC tool.

23. As part of strengthening the legislative, regulatory and institutional framework relating to public-private partnerships (PPPs), the Government undertook to review Law No. 020-13/AN of 23 May 2013 on the PPP legal regime in Burkina Faso and its implementation instruments to mark, 
among other things, the difference between a PPP and a conventional supplier credit. Accordingly, a diagnostic report on the current legal framework was drawn up on the basis of the recommendations from technical assistance on the one hand and, on the other hand, guidelines on PPP support strategy and the draft WAEMU directive. This diagnosis made it possible to identify the main principles and thrusts that will enable a review of the PPP development strategy adopted since 2011. The strategy review has begun and its adoption will help begin the review of the law, the adoption of which is envisaged in 2020. However, pending adoption of the new PPP law, the Government took measures in 2018 to give preference to concessionary PPPs on the one hand and, on the other hand, to ban PPPs likened to State pre-financing and guarantees.

24. In the energy sector, efforts continued to increase electricity production and raise the share of renewable energies in the energy mix. Thus, the installed electric power, estimated at $324.6 \mathrm{MW}$ in 2017 hit $351.7 \mathrm{MW}$ in 2018 due to the increase in electricity import on average of $60 \mathrm{MW}$ through the Bolgatanga (Ghana) and Ouagadougou (Burkina Faso) interconnection. Added to this is the production of 33 Megawatts Peak (MWp) by the Zagtouli's solar photovoltaic power plant which boosted renewable energies, whose share of total production increased to $13.99 \%$ in 2018 compared with $12.53 \%$ in 2017 . Regarding rural electrification, several achievements were made in 2018 including (i) completion of electrification of 20 rural localities by the Electrification Development Fund (EDF) and (ii) electrification of 20 localities under the project for the electrification of 100 localities by hybrid or isolated photovoltaic solar system. Regarding the promotion of renewable energy and energy efficiency, there is a significant increase in the share of total production of renewable energy from $6.4 \%$ in 2015 to $13.99 \%$ in 2018.

25. Like other countries in the sub-region, notably Mali and Niger, Burkina Faso is experiencing an increasingly difficult security environment. Although being preserved for long, the country entered a cycle of increasingly frequent terrorist attacks resulting in civilian and military casualties, the closure of some schools located in exposed areas, the destruction of public property (schools, police and gendarmeries stations) and internally displaced populations fleeing conflict zones. Faced with this security challenge, the Government has initiated a reorganization of the defence and security forces resulting in the strengthening of their operational capacities through an increase in the number of personnel, the acquisition of efficient equipment, the intensification of intelligence and the construction and/or completion of the construction of District Police Stations and Territorial Gendarmerie Brigades. Some major investments have also progressed in the sector. Worthy of note is the institution of the electronic passport system (e-passport), the continuation of the project to secure Burkina Faso visas (PSV-BF) and the police identification, rapprochement and analysis project (IRAPOL).

In December 2018, a state of emergency was declared in several provinces in the north and east of the country with an intensification of the offensive of the national armed forces on the ground. In January 2019, the ministers of defence and security were replaced following a cabinet reshuffle. Since February 2019, the President of Burkina Faso, Roch Marc Christian Kabore, is heading the rotating presidency of G5 Sahel. 
26. As part of the pooling of forces and the fight against terrorism, from 18 February to 1 March 2019, Burkina Faso hosted Operation Flintlock 2019, a US-backed multinational military exercise involving more than 2,000 military personnel from more than 30 African and Western partner countries.

27. In 2018, in the health sector, public action led to an increase of health infrastructure and the improvement of their equipment. The main achievements include: the equipping of the University Teaching Hospital (Sourou-Sanon and Yalgado-Ouédraogo) and Regional Hospitals (Kaya, Gaoua, Dori, Koudougou and Fada N'Gourma) in autonomous oxygen production system; (ii) the transformation of 23 CSPS in the chief towns of rural councils into medical centres; (iii) the construction and equipping of a haemodialysis centre in Bobo-Dioulasso; (iv) the transformation of the Ouahigouya Regional Hospital into a University Teaching Hospital; (v) the construction of the Dédougou Regional Hospital; (vi) the construction and equipping of 93 CSPSs.

28. In the field of education, one of the main achievements is the increased educational and administrative infrastructure at all levels of education. In primary education, the efforts of the State and other actors made it possible to increase the number of primary schools to 15,756 including 3,985 private schools in 2017/2018 as against 14,655 including 3,363 private schools in 2015/2016, a $7.5 \%$ increase, thus encouraging an $11.6 \%$ increase in primary enrolment. Regarding post-primary and secondary education, as of 31 December 2018, new classrooms were built for high schools and colleges that increased the number of post-primary and secondary schools to 3,810 in 2017-2018 compared with 3,282 in 2016-2017, an 15\% increase with a $10.21 \%$ increase over the same period.

29. Actions in the field of employment focused on strengthening the legal and institutional framework of decent work through the review of Law No. 028/2008/AN to institute the Labour Code and the promotion of the socioeconomic reintegration of laid-off and retired workers. In addition, 84 micro projects were funded from a forecast of 100, increasing the number to 318 micro projects at the end of December 2018. Implementation of the Youth Employment Program for National Education (PEJEN) made possible the recruitment of 1,659 young people out of 2,100 young university graduates envisaged in 2018.

30. As part of the social dialogue, in terms of investments, the Government rehabilitated the Ouagadougou Labour Exchange and took other actions such as support for social partners in concluding collective agreements (BRAKINA -SODIBO, mines, banks and financial institutions and security companies), settled 6,930 individual labour disputes out of 7,084 recorded in the Regional Directorates of Labour and Social Protection.

\section{B. Program Implementation at End-December 2018 and End-March 2019}

31. Almost all program quantitative criteria were met at end December 2018 and at end March 2019. Indeed, of the five quantitative performance criteria, only one was not met, namely the criterion on the net domestic financing of the State that stood at CFAF 394.59 billion at the end of December 2018 with the inclusion of SONABHY securities and CFAF 71.23 billion at the end of March 2019 for a ceiling set CFAF 258 billion and zero respectively. Non-concessional external debt 
contracted or guaranteed by the State amounted to CFAF 56.42 billion at end December 2018 against a ceiling of CFAF 200.00 billion. At end March 2019, for a ceiling of 370 billion FCFA, the external debt contracted or guaranteed by the State (in actual value) amounted to CFAF 6.07 billion. In addition, the State did not accumulate external arrears or guarantee domestic loans to suppliers and contractors. Lastly, the State did not guarantee bank pre-financing for public investment.

32. The transition from classic to program budgeting in 2017 required a learning process for the appropriation of this new programming approach by the sectoral ministries despite the support provided by the Directorate General of the Budget. As a result, the first half of 2017 witnessed a very low level of engagement of budgeted spending as the government had just launched the Emergency Program for the Sahel (PUS-BF). The PUS-BF includes major investment projects for the northern regions heavily affected by terrorism, with an overall budget of about CFAF 455 billion for the 2017-2020 period. This program has been extended to new fragile regions (Boucle du Mouhoun, East, Center-East, and Center-North). Thus, in September there was an acceleration of commitments to make up for the delay accumulated earlier in the year and to avoid being foreclosed by the cessation of commitments at the end of October. This led to a significant accumulation of nonmandated committed expenditures (DENO) of approximately CFAF 110 billion at the end of December 2017.

33. It should also be noted that, in 2017, the government, in view of the widespread social unrest in general and at the MINEFID in particular, had requested and obtained from the National Assembly authorization to streamlining procedures to allow the execution of large PNDES projects. This enabled an acceleration of the procurement process, the first tranches of which were liquidated at the end of December because of the multi-year period, resulting in a significant accumulation of DENO of around CFAF 110 billion at the end of December 2017.

34. To avoid recurrence of such a situation in the future, better monitoring of investment programming is now in place. Now that the implementation of the program budgeting tool is getting under a better control by budget officers, better planning and spreading of commitments throughout the year should help avoid large accumulations of DENO. In addition, we will assess with the IMF Resident Adviser during its visit in October 2019 the adequacy of our expenditure tracking systems.

35. All indicative targets were met or exceeded: (i) there were no domestic arrears at end December 2018 and end March 2019; (ii) overall budget deficit including grants stood at CFAF 381.74 billion at end December 2018 for a ceiling of CFAF 382 billion; (iii) collected own revenue stood at CFAF 1,530.78 billion at end December 2018 and CFAF 396.42 billion at end March 2019 for ceilings set at CFAF 1,444 billion and CFAF 376 billion respectively; (iv) current social expenditures for poverty reduction were executed to the tune of CFAF 204 billion for a ceiling of CFAF 176 billion at end December 2018, and to the tune of CFAF 73 billion at end March 2019 for a ceiling of CFAF 48 billion; $(v)$ the stock of more than 30 days certified and unpaid VAT reimbursement stood at CFAF 26.8 billion against a ceiling of CFAF 55 billion at end December 2018 and at CFAF 44 billion at end March 2019 for a ceiling of CFAF 45 billion and (vi) no PPP project was contracted at end December 2018 or end March 2019. 
36. In 2018, out of the 7 (seven) structural benchmarks remaining to be implemented, 4 (four) have been met while 3 (three) are still in progress. These include benchmarks for: (i) the development and adoption of a single treasury account (STA) operating mechanism; (ii) the recruitment of an independent external auditor to audit SONABHY's financial operations and accounting practices; and (iii) digitalization of the declaration of property of the persons concerned by the law and establishment of an online database (online statement, online consultation).

37. Regarding the single treasury account (STA), an IMF technical assistance mission was conducted in Ouagadougou from 15 to 26 April 2019 after another mission in January 2019 and focused on the review of shares. The mission ensured quality control on the technical and regulatory documents of the STA and a roadmap for its implementation. A training and awareness workshop was held for actors at national level, including BCEAO. A focal point has been designated and a note establishing the STA steering committee is in the process of being signed. The technical support for setting up the STA (connection to STAR and SICA through SWIFT) is being acquired with World Bank support.

38. With regard to the recruitment of the auditor of SONABHY, the firm that had been shortlisted withdrew after several months of negotiations that resulted in an agreement on the technical and financial bid. Currently, an invitation has been sent to the firm that ranked $2^{\text {nd }}$ and deemed qualified during the evaluation of the expressions of interest. The latter submitted its technical and financial proposal on Wednesday, 24 April 2019. The committee in charge of analyzing the proposal approved it and the file was sent to the funding body for a no-objection opinion.

39. Regarding the measure relating to the digitalization of asset declarations, many actions have been carried out. Indeed, consultants have been recruited for the development of the platform, the quality review, the delivery of equipment and the setting-up of the website. The specifications for the platform have also been drafted. The website has been created and is currently hosted by the National Agency for the Promotion of Information and Communication Technologies (ANPTIC) pending the delivery of ASCE-LC servers. ASCE-LC had planned (i) the development of the platform for the declaration of interests and property at end March 2019 and (ii) the installation of the server platform plus the local network overhaul and the standardization of the computer room plus its security at end May 2019. Unfortunately, given the delays experienced by the recruited service provider, these deadlines can no longer be respected.

40. For 2019, 14 (fourteen) benchmarks were selected for implementation. All 4 (four) measures scheduled to be implemented during the first quarter of 2019 were realized. Two measures were due by the end of April 2019. Of these two, one has been completed and the other is in progress. The one currently being executed concerns the publication of the number and distribution of civil servants by category, rank, region and ministry. At this level, data has been collected and processing is in progress.

41. One measure is to be carried out by end June 2019. It relates to the establishment of a special account funded by a portion of the VAT revenue and dedicated to refunds of VAT credits 
and the quarterly production of a report on the operational management of this account. This measure is at an advanced stage of implementation.

42. The 6 (six) measures to be carried out during the second half of 2019 are generally being implemented satisfactorily and will have to be completed by the end of the year.

\section{IMPLEMENTATION OF PNDES}

43. Implementation of the PNDES in 2018 took place in a very difficult security context and a social front marked by particularly numerous wage demands. Indeed, there has been persistent wage demands and an upsurge in terrorist attacks in many parts of the country. The end of the year was moreover characterized by brief episodes of community tensions. Despite this difficult context, the implementation of the PNDES is highly appreciable. Highlights of the assessment of implementation of the policies operationalizing the PNDES in 2018 are presented by strategic thrusts.

44. With regard to institutional reform and the modernization of the administration (thrust 1), the main results obtained include strengthening of the operational capacities of the defence and security forces.

45. In the areas of human rights and justice, the major gains are in terms of increasing the financial accessibility to the justice system, through the Legal Aid Fund to covering the court costs of 297 people, including 111 women in 2018. They also concern the expedited processing of disputes, the humanization of the prisons, the strengthening of the judicial system with regard to judicial personnel, court officers and human rights specialists.

46. Regarding the promotion of good economic governance, the major achievements in the planning and management of the economy relate to: (i) adoption and implementation of the law on steering of the economy and development management; (ii) operationalization of the new financing instruments of the economy, namely the Bank for Agricultural Financing, known as the Agricultural Bank of Burkina Faso (BADF) and the Deposits and Consignment Office (Caisse des Dépôts et de Consignation - $(D C)$, and (iii) reinforcement of the accountability of public expenditure actors with the accentuation of audits of public structures as well as ASCE-LC investigations into suspicions of malpractices.

47. In human capital development (thrust 2), the continuation of the policy of free healthcare for pregnant women and children aged less than 5 years has lifted the economic barrier of access to quality care by offering a total of 17,861,499 acts of free care at a total cost of CFAF 31,701,313,632. In addition to free medical care, efforts have been made to increase the number of medical and paramedical personnel, to provide the Medical Centers with Surgical Antennas (CMA) with specialized health personnel and to continue building health centres and/or equipment, maintaining prevalence of HIV in the general population below $1 \%$, lower rates of discontinuance of contraceptive products, increased couple-year protection (32\% as against $27 \%$ in 2017 ) and the establishment of the National Pharmaceutical Regulation Agency (ANRP). 
48. At the level of education, efforts to improve the supply and quality of education have been pursued with the expansion of educational and administrative infrastructures at all levels of education; recruitment of teachers and/or trainers and improving students' and learners' access to scholarships and to the school canteen that helps keep many of them in the education system. These achievements led to an increase in (i) the post-primary and secondary completion rate, which was increased to $40.3 \%$ from $32.95 \%$ in 2017 , and (ii) the proportion of students enrolled in vocational training branches suitable for the structural transformation process, which is $45.8 \%$ for a target of $20 \%$.

49. With regard to drinking water, relatively significant gains have been made in terms of access by urban and rural populations to sources of drinking water, the implementation of Simplified Drinking Water Supply Systems (AEPS), autonomous drinking water supply points, new equipped boreholes including 2,491 in rural areas, Human-Powered Pumps (PMH) and rehabilitation of water and sanitation infrastructure. Achievements have made it possible to increase the national rate of access to drinking water from $73.4 \%$ in 2017 to $74 \%$ in 2018 and to increase the storage and water production capacity of the National Water and Sanitation Board (ONEA) at $450 \mathrm{~m}^{3}$ and $7658 \mathrm{~m}^{3}$ per day.

50. In order to boost the high-potential economic growth sectors and jobs (thrust 3), major achievements include improving the cereal coverage rate and improving the coverage rate of livestock feed requirements by $115 \%$. Notable results have been achieved as concerns road infrastructure. These include the completion of $171 \mathrm{~km}$ of tarred roads and the construction of 660 $\mathrm{km}$ of rural roads, thus increasing the proportion of developed rural roads to $36.5 \%$ for a target of $32.6 \%$ in 2018. In addition, significant bridges have been completed and commissioned, namely the bridges over River Mouhoun and River Sirba. In terms of industrial development, the major reform concerns the adoption of Law No. 038-2018 to institute the Investment Code and implementation of the SME Promotion Orientation Law through the signing of the Small and Medium Enterprises (SME) Charter.

51. In terms of improving Internet access, the major gains are the increase in the Internet bandwidth, which rose from $16 \mathrm{Gbps}$ in 2017 to $39.3 \mathrm{Gbps}$ in 2018 and Internet use rate in the last 12 months estimated $31.26 \%$ in 2018 as against 24\% in 2017. These results were made possible by the continuation of the provinces' fibre optic interconnection work (National Backbone), with the deployment of $295 \mathrm{~km}$ of optical fibres.

52. The gains also relate to the increase in installed electric power and the solar photovoltaic power supply of several villages and socioeconomic infrastructures.

\section{ECONOMIC AND FINANCIAL POLICIES FOR THE REST OF 2019 AND FOR 2020}

53. Government intends to maintain its efforts to support growth while taking the necessary measures to stabilize the macroeconomic framework. 


\section{A. Macroeconomic Framework}

54. Economic activity is expected to slow down in 2019. Indeed, the real GDP growth rate is projected to decelerate to around 6\% in 2019 compared with $6.8 \%$ in 2018 mainly due to a normalization of agricultural production. Inflation is expected to remain within the limit of the Community standard of $3 \%$ maximum in 2019 , in connection with the pursuit of consumer goods price control measures and the good prospects of the 2019/2020 agricultural season.

55. Foreign trade in 2019 is projected to be marked by increased imports and exports, in connection with the good orientation of domestic economic activities. The adverse impact of higher oil import bills and cotton fibre exports would contribute to the deterioration of the trade balance, despite an expected rise in the world price of gold, combined with the expected growth of mining production. However, it is expected that net capital inflows and net financial flows will improve with the rest of the world.

56. For 2019, the current account deficit is expected to deteriorate to $6.3 \%$ of GDP, mainly in line with a higher increase in imports.

\section{B. Fiscal Policy}

57. The Government will continue its efforts to consolidate public finances and implement the reforms needed to increase revenue mobilization and strengthen expenditure monitoring. Thus, reform measures for revenue enhancement and expenditure management will continue and intensify.

58. Revenue mobilization will continue to be supported by the measures outlined in the 12 December 2018 Memorandum of Economic and Financial Policies. These include the following:

- broadening the scope of the tax on gains the on the issuance of mining permits;

- imposition of a tax on luxury vehicles;

- raising the rates of the arms tax;

- raising the tariff of levies on air tickets;

- broadening the scope of the liquor licence;

- abolition of the flat-rate system of taxation of activities of the beverages sector and their submission to the common-law tax system with institution of a $5 \%$ levy as deposit of the tax on profits;

- raising the tax rate on non-alcoholic beverages from $10 \%$ to $15 \%$;

- raising the rate of the tax on biodegradable and authorized non-biodegradable plastic packaging and plastic bags;

- improving the yield of the Property Tax on Buildings and Unbuilt Land (TFB/NB), the Residence Tax (TR) and the Micro Enterprises Contribution (CME) thanks to data from the general census of Ouaga and Bobo taxpayers;

- the expected performance in the collection of outstanding amounts;

- strengthening audit in offices by activating the ASYCUDA value module; 
- continuous monitoring of the valuation of goods in the offices by the Value Control Unit;

- the continued outsourcing of CCVA's evaluation of used vehicles under ten years old and public works machinery with the reduction of the discount to $25 \%$ of the value instead of $50 \%$.

59. In addition to these measures, most of which are included in the 2019 Finance Law, the following steps will be implemented:

Under the DGI, the implementation of the new features (e-services) in e-sintax [launched on 28 May 2019]. The new e-sintax version will have the following features:

$>$ online declaration and online payment of contract registration fees to enable the online publication of tax settlement slips;

$>$ online payments via mobile money in partnership with Orange Money;

D DGI cash: cash payments of online declarations in banks in partnership with UBA;

$>$ online VAT refund claims;

$>$ issuance of tax status certificates and turnover certification online.

Under the Directorate General of Customs, the submission of 28 (twenty-eight) other products to the control value according to the following timeline: 8 (eight) at end June and 20 (twenty) in the second half of 2019, 50 (fifty) risk products controlled in ASYCUDA and monitored by the value control team.

60. At the end of March 2019, the Directorate General of Taxes (DGI) recorded outstanding receivables (RAR) amounting to CFAF 26.32 billion due to a lack of follow-up caused by a strike action of workers of the ministry in charge of finance. To bridge this gap, the DGI intends to conduct a major recovery operation from 3 to 30 June 2019, which will essentially consist of systematic reminders to the defaulting taxpayers of the Department of Large Companies and the three (03) Medium-sized Enterprises Departments. The objective of this operation is to ensure timely declarations and payments of all taxes, and to restore the pre-crisis situation.

61. The implementation of all these measures will enable the projection of revenue and donations in 2019 estimated at CFAF 1,945.4 billion against a realization of CFAF 1,745.9 billion in 2018 , i.e. an $11.4 \%$ increase. The implementation of measures relating to broadening of the tax base and new taxes will improve the tax burden rate, which is expected to be around $18 \%$.

62. Expenditure forecasts for total expenditure and net lending are maintained at CFAF 2,204.14 billion in 2019 compared with CFAF 2,127.61 billion in 2018. This 2.3\% increase in total expenditure is mainly attributable to staff costs, which stand at [9.9\%] of GDP compared with $9 \%$ in 2018. Capital expenditure on own resources will be [4.1\%]. Total spending and net lending are expected to account for $25.2 \%$ of GDP, down by 1.8 percentage points from 2018 . 
63. Measures to pursue the streamlining of recurrent expenditures will include:

- adoption and implementation of a policy to optimize the vehicle fleet of the State;

- restriction of the relocation of activities;

- effective implementation of stores accounting;

- better monitoring of electricity, telephone and water consumption.

64. In order to strengthen the effectiveness of fiscal management, the following actions will be carried out:

- anticipation of the procurement process and actions aimed at reconciling the physical and financial execution of the budget, particularly in the context of delegated project management;

- commissioning of program managers for a better steering of public action;

- the continuation and strengthening of legal and regulatory expenditure procedures (exante and ex-post) including security-related procedures;

- institution of a mechanism to monitor and control physical achievements on the State budget, at regional level.

65. The overall deficit in 2019 is expected to be $3 \%$ of GDP, down by 1.9 percentage points from its 2018 level. This deficit level will continue in 2020, in line with our commitments under our IMFsupported ECF program and the WAEMU convergence criteria. It will be financed in 2019 and 2020 through fiscal support, drawings on IMF resources and issuance of securities on the financial and monetary markets.

\section{Debt Management Policy}

66. Burkina Faso's medium-term debt management strategy aims to keep the risk of debt distress at a moderate level. To this end, it plans to reduce domestic and regional borrowing and resort more to external financing sources that have lower costs and more favourable terms for the next two years. The government will pursue a prudent debt policy to maintain its moderate risk of external debt distress. As in previous years, the objective of debt management remains meeting the State's financing needs. The aim is to enable the State meet its payment obligations at the lowest possible cost in the long term, by keeping the risks to a satisfactory level and by achieving other objectives such as the development of the sub-regional financial market. It is also necessary for the Government to monitor and contain the accumulation of contingent liabilities by streamlining State guarantees. In a context of reduced concessional resources, the use of non-concessional financing will be an alternative for projects with high and guaranteed economic return.

67. The outstanding public debt at 31 December 2018 stands at CFAF 3,365.95 billion, made up of CFAF 1,456.39 billion for domestic debt and CFAF 1,909.56 billion for external debt, i.e. 43.27\% and $56.73 \%$ respectively. The debt ratio (outstanding debt/nominal GDP) at this date is estimated at 42.9\% against 38.4\% at 31 December 2017. 
68. At end March 2019, the provisional stock of public debt amounted to CFAC 3387.66 billion. It is composed of CFAF 1424.92 billion domestic debt and CFAF 1962.73 billion external debt, corresponding to $42.06 \%$ and $57.94 \%$ respectively of the public debt.

69. In terms of drawings, CFAF 146.50 billion of external debt was mobilized at 31 December 2018. At the level of the domestic debt, an amount of CFAF 704.7 billion was mobilized on the financial market over the same period, including CFAF 347.2 billion for bonds and CFAF 357.5 billion for Treasury bills.

70. As at 31 March 2019, the drawings were composed of CFAF 117.864 billion of domestic debt (equivalent Treasury Bills and Treasury bonds) and CFAF 36.51 billion of external debt.

71. The ceiling for external debt contracted or guaranteed by the State in 2019 is CFAF 370 billion in net current value. For 2019, the government will resort to government issuance of securities on the regional financial market as well as a combination of concessional and nonconcessional borrowings. In addition, the Government remains committed to achieving the targets set in the program for the budget deficit of 3\% of GDP in 2019.

72. As part of the debt management strategy, the Burkina Faso authorities plan to replace the maturing domestic debt with concessional external borrowing. The proceeds from these operations will be used solely for debt and cash management purposes and not to increase overall expenditures beyond the 3\% of GDP limit set by the program in 2019. Before embarking on such a debt management operation, Burkina Faso undertakes to consult with IMF staff and to provide detailed information on financing conditions.

73. In the area of debt management capacity building, brainstorming and actions are ongoing in view of improving the efficiency and effectiveness of good practices and standards in the field, drawing in particular on the recommendations of technical assistance missions of specialized agencies. In this light, actions are underway to improve the design and publication of the debt statistical bulletin.

\section{STRUCTURAL REFORMS FOR THE REST OF 2019 AND 2020}

74. The economic and financial program will continue to be backed by the continued implementation of structural reforms.

\section{A. Public Finance}

75. Burkina Faso has completed an assessment of its Public Financial Management (PFM) system based on the Public Financial Management Performance Measurement Framework (PEFA). PEFA notes that the performance of Burkina Faso's PFM system is still insufficient to meet the three fiscal objectives of fiscal discipline, strategic resource allocation and effective and efficient public service delivery. A first action plan to address the challenges identified by the evaluation was developed for the period 2018-2020 and has been relatively weakly implemented due to lack of resources and weak ownership by some actors. This action plan has been updated for 2019-2021 and will be 
reviewed again to incorporate the results of other evaluations such as the tax administration evaluation diagnostic tool (TADAT), the public investment management evaluation (PIMA) and the Debt Management Performance Assessment Instrument (DeMPA).

76. The authorities undertake to implement the single treasury account (STA). In a context of scarcity of resources, the implementation of the STA is a major challenge in order to drain public liquidity, available in a multitude of bank accounts, to a Treasury Regulation Account (TRA). By centralizing public funds in this way, the State cash flow manager is able to meet the State's commitments by optimizing the cost of borrowing, and can, where necessary, invest possible temporary surpluses. In view of these challenges, the implementation of the STA is a key measure. In order to ensure the implementation of the STA, we will (i) conduct the census of public accounts in commercial banks, (ii) close inactive accounts, and (iii) repatriate their credit balance to the STA. At the same time, we plan to adopt a decree setting the perimeter of the STA, and an order to lay down conditions of its opening and functioning (structural benchmark).

77. Implementation of the STA and the public bank accounts monitoring system must be based on good quality accounting, produced on time. For this purpose, the production of a general balance of accounts is an essential prerequisite in order to reconcile the balances of the availability accounts of the general accounts with the balances of the statements of account transmitted by $\mathrm{BCEAO}$ and commercial banks. The process of transitioning to accrual accounting is underway.

78. Actions will be carried out to continue the implementation of the program budget, which began in 2017. These include:

- the continued adaptation of the IT system of financial services in order to integrate new modules in view of greater efficiency;

- finalization of the review of the budget programs template;

- continued empowerment of stakeholders through the appointment of all program managers;

- determination of the State property: establishment of the $1^{\text {st }}$ opening balance sheet, effective keeping of stores accounting;

- review of some guidelines and templates (monitoring-evaluation, Annual Performance Project, Annual Performance Report ...);

- continuing reforms to improve the process of maturation, evaluation, execution and monitoring and evaluation of investment projects;

- continued efforts for the streamlining of the expenditure chain and putting in place of initial expenditure programming tools as well as performance management tools;

- publication of the budget in the form of functional nomenclature.

\section{B. Containment of the Wage Bill}

79. In order to reduce personnel expenditure, in percentage of tax revenue, to the level required by the regional directives (UEMOA) in the medium term, several actions will be carried out: 
- clearing of arrears in respect of pecuniary benefits owed staff over previous financial years, in order to comply with the annual requirements of the budget;

- intensification of the internal control of remunerations granted to State employees;

- continued cleaning of the payroll, coupled with the biometric enrolment of the State employees;

- continued implementation of the conclusions of the national conference on the remuneration system of government employees, in particular the elaboration of the related legislative and regulatory texts (organic law, salary indexation and indemnity scales). Already, actions are envisaged to redeploy the workforce, particularly in the education sector, which accounts for about $60 \%$ of civil servants, and to adopt a text to regulate the salaries of top public personalities;

- preparation by end-September 2019 of the medium-term strategy document on public service remuneration (organic law, salary and allowance scales) and recruitment, consistent with the WAEMU convergence criteria;

- implementation of a communication policy on the wage bill strategy, at the highest level, to raise awareness about disproportionate increases in the wage bill that could crowd out other types of spending, in particular for security and investments. This includes a public declaration by the government on its commitment to gradually reduce the ratio of the wage bill to tax revenues from 2020 onward (prior action); In this context, the Government intends to organize very soon a stock-tacking conference on the public agents remuneration system;

- non-completions of new agreements resulting in the increase of the wage bill prior to adoption of all the reforms intended to contain the wage bill, including the organic law on the civil service and the related implementation instruments.

\section{Pump Price Adjustment Mechanism}

80. With regard to the pump price adjustment mechanism that was put in place in 2018 , the authorities pledge to continue efforts to improve its operationalization. The government intends to maintain a policy aimed at ensuring the sustainability of SONABHY, safeguarding its mission of ensuring adequate supplies, and reducing the hydrocarbon subsidy, in order to release more resources for enhancing investments and supporting the social sectors. To this end, the operationalization of the mechanism will be delegated to a technical structure that automatically communicates the new real prices to the public at the beginning of each quarter (starting July 2019). Already, the implicit subsidy for fuel prices at the pump is published monthly on the website of the Ministry of the Economy, Finance and Development.

\section{Improving the Performance of the Energy Sector}

81. The effective implementation of the Memorandum of Understanding between the State of Burkina Faso, SONABEL and SONABHY relating financial relations and fixing of hydrocarbons sale price to SONABEL, in particular the points concerning the partial payment of SONABEL's debt to 
SONABHY and the methods for determining the price of hydrocarbons improved the financial situation of SONABEL, which resulted in:

$>$ profits registered from 2016 to 2018;

$>$ compliance with commitments to suppliers and TFPs;

$>$ clearance of all of reimbursement arrears to the State;

$>$ increase in SONABEL's equity and hence improvement of its financial structure.

82. With regard to SONABHY, the measures taken by the Government to settle its debt enabled it to record a 43.13\% profit in 2018 compared with 2017. In accordance with the 2019 Finance Law, the government will settle the remaining debt due to SONABHY in 2018 which was not yet securitized and amounted to CFAF 64.6 billion.

\section{E. Cotton and Other Agricultural Sectors}

83. In the cotton sector, the replenishment of the flattening fund and the operationalization of the cotton inputs fund helped to consolidate its financial situation. In addition, the Government, in coordination with cotton producers and cotton companies, intends to continue its strategic support to increase the productivity and quality of cotton and also promote diversification. The sector will have to pursue innovative reforms in order to implement strong measures (constant improvement of the sector's management tools, adaptation to the effects of climate change, improvement of good farming practices, etc.) for the sustainable revival of cotton production in Burkina Faso, which has underperformed in the last two seasons. Implementation of the strategic plan resulting from the national workshop on the sustainable revival of cotton production held on 11, 12 and 13 March 2019 in Ouagadougou will contribute significantly to achieving the goal of a sustainable revival of national cotton production. Accordingly, the Government intends to support private or public initiatives to increase the value added of cotton, through the local processing of Burkina Faso's fibre and thus create more jobs for young people and women and wealth for the entire nation.

84. Moreover, to ensure the development of a productive and resilient market-oriented agroforest-pastoral, wildlife and fisheries sector, the Government has taken actions geared, among others, toward (i) the scale of innovative mechanisms to improve the delivery of the Agricultural Council; (ii) the institution of a new mechanism for subsidizing inputs; (iii) the creation of an innovative incubator of agricultural entrepreneurs at the level of some Centres for Rural Development (CPR) and the Multipurpose Agricultural Centre (CAP-Matroukou); (iv) acceleration of agricultural mechanization through the provision at subsidized prices of agricultural equipment and the opening of a tractors and tillers assembly plant; $(v)$ the promotion and the development of innovative mechanisms for access to agricultural credits such as agricultural insurance, agricultural storage and warrantage; (vi) pursuit of agricultural cooperatives compliance with the OHADA Uniform Act; (vii) decentralization of the agricultural bank of Burkina Faso to bring its services closer to producers; (viii) actions taken to improve the business climate in the agricultural sector and signing of a technical assistance agreement with China for the construction of the major irrigation facilities and provision of agricultural consultant support services; (ix) in the Sourou Agropole, 
continued implementation of irrigation development projects; $(x)$ continuation of work on the Sanghin dam under the PVEN project with AGETER; (xi) construction of agricultural produce processing plants in high production areas; (xii) building of post-harvest infrastructure (canneries, counters, warehouses); (xiii) setting up of agricultural development fund, awaiting adoption by the Council of Ministers of the decree to establish the fund; (xiv) construction of a natural phosphatebased mineral fertilizer production plant; (xv) the creation of an agricultural training centre in each of the regions of the Centre, Central plateau, North, East-Central and Cascades; (xvi) the establishment of the national agency for rural land (ANTR) whose preparatory documents have been developed and the process is ongoing.

\section{F. Financial Inclusion}

85. Financial inclusion is one of Government's top priorities. One of the objectives of PNDES is to increase the bank use rate (total population base) to $35 \%$ by 2020 . In this light, the Government has undertaken to develop a National Inclusive Finance Strategy (SNFI) with an implementation action plan. The SNFI was adopted by Decree No. 147/MINEFID/CAB/SP-PMF of 23 April 2019 in accordance with Decree No. 2018-0775 relating to the management and steering of development in Burkina Faso. In addition, for its operationalization, there are plans to set up a Monitoring Committee, a Technical Secretariat and working groups. Given the scarcity of internal resources, a round table is planned to mobilize funding from development partners. The process of organizing the round table has begun. With regard to promoting access to credit, there has been some developments. First, the law No. 013-2016/AN of May 3, 2016 on the Credit Information Bureau (BIC) was adopted and promulgated by Decree No. 2016-425/PRES regulating credit information bureaus in Burkina Faso, which allowed the operationalization of a private credit bureau. However, it became necessary to remove the obligation to obtain the prior consent of the customer on the old loans by modifying article 53 of the law $N^{\circ}$ 013-2016/AN of May 03, 2016 to allow the functioning of the BIC and a significant increase in its client database in Burkina Faso.

86. This amending Law was adopted by the National Assembly in its session of May 14, 2019. Secondly, an agricultural bank became operational in February 2019 and aims to reach the underserved agricultural sector. Lastly, the operationalization of the Deposits and Consignment Office (CDC) continued to advance with the appointment of the Director General and Central Cashier in April and May 2019, respectively. Like the agricultural bank, the CDC aims to fund underserved market segments with a special focus on local governments, small- and medium-sized enterprises and low-cost housing.

\section{G. Poverty Reduction}

87. To substantially increase access by the population to quality health services, the main actions envisaged in short and medium term aim to (i) reduce maternal mortality rate, infant and child mortality rate, as well as the death rate of severe malaria among children and (ii) reduce the HIV prevalence rate to less than $0.77 \%$. To this end, the free health care reforms benefiting children aged from 0 to 5 years, pregnant women, destitute elderly persons, as well as the reform on free family planning will be continued. 
88. To help improve the nutrition of women and children in particular, interventions will aim to provide $95 \%$ health care for cases of severe malnutrition and increase to rate of severe malnutrition cure to $100 \%$.

89. In order to promote decent employment and social protection, government will take actions to: (i) raise the professional integration of young people and women to $20 \%$ through the employment promotion system; (ii) reduce the proportion of children aged from 5 to 17 years involved in economic activities to $25 \%$.

90. As part of the promotion of the national health insurance system for all, activities in 2019 will focus on the functioning of the main health insurance bodies and the beginning of CNAMU transfers, procurements and control of free health care for women and children aged less than five years.

91. In order to reduce social and gender inequalities and to empower women as dynamic development actors, the main actions to be implemented will aim to: (i) integrate at least $75 \%$ of identified marginalized persons; (ii) increase to at least $50 \%$ the proportion of active disabled persons employed; (iii) support at least $70 \%$ of identified vulnerable children; (iv) rehabilitate approximately 2,860 street children and $(v)$ increase to $50 \%$ the proportion of female entrepreneurs.

92. In order to improve living conditions, access to drinking water and sanitation, it will be necessary to continue efforts to raise the national rate of access to drinking water to $79 \%$ and increase the rate of sanitation to $34 \%$ in 2020.

93. Actions to support the resilience of households in the agricultural sector will be maintained and intensified in 2019 and 2020 with the aim of reducing the proportion of non-resilient households to less than $5 \%$ and that of households with structural vulnerabilities to less than $5 \%$. In addition, efforts will be made to increase the coverage of cereal needs to $140 \%$ per year.

94. In order to develop local economies and reduce disparities, Government's future policies will aim at continuing the implementation of the Sahel Emergency Program (PUS-BF), which will be extended to other regions and the Local Economies Development Support Program (PADEL) in six new regions.

In Burkina Faso, social protection is a fundamental priority that is taken into account in one of the objectives of the National Economic and Social Development Plan (PNDES) entitled "to promote decent employment and social protection for all, particularly for the elderly, young people and women ".

95. To this end, the Government has taken measures and put in place a social protection system covering two aspects: social safety nets and social insurance.

Assistance to people in social vulnerability includes cash transfers, in-kind transfers, subsidies and exemptions, and labor-intensive work. 
About 102,500 vulnerable households in the North, Central East, and East, Central West and Sahel regions benefited from cash transfers under the project. The amounts of cash transfers are CFAF 30,000 to CFAF 40,000 per quarter, depending on the number of young children in beneficiary households ( 0 to 15 years).

96. In-kind transfers mainly involved free distributions of food (more than 40,000 tonnes to at least 400,000 vulnerable people in the provinces affected by the cereal deficit of the agricultural campaign), subsidized food sales organized by the SONAGESS $(27,852$ tons of food to 89,506 vulnerable households as part of the food insecurity response plan) and school kit distributions.

97. In addition, action is being taken, in collaboration with partners, to support more than 25,122 refugees, the majority of whom come from Mali, and 492,725 internally displaced persons due to terrorist attacks. Thus, in order to assist people with social vulnerabilities, and to pursue the social safety net project, the government plans to strengthen its actions by directly transferring subsistence credits in the amount of CFAF 9.6 billion to 86,616 beneficiaries from poor and vulnerable households in all regions targeted by the project in 2019, an increase of $36.19 \%$ compared to 2018. To this end, the project envisages to start the administrative formalities for its intervention in the regions of Boucle du Mouhoun, the Center-North and the province of Sanguié in the Center-West. In addition, the first payments will take place in the Sahel region.

\section{H. Improvement of Macroeconomic Data}

98. Government has continued its efforts to improve macroeconomic data. The findings of the national survey on employment and the informal sector as well as those of the study on artisanal gold mining have been included in the national accounts for the new baseline year 2015 in accordance with the 2008 SCN. The findings of this new baseline were finalized and validated during an international technical workshop organized in November 2018 and bringing together several specialists from national and international structures (IMF, World Bank, European Union, AFRISTAT, WAEMU in particular). The findings are published on the INSD website.

99. Following the rebasing of the national accounts, work is underway to revise the retrospective series with a view to ensuring the overall coherence of the macroeconomic aggregates. The revised demand-side GDP series up to 1999 is available. The production-side series is being finalized and all retrospective series will be published by September 2019.

100. Still in view of continued improvement in macroeconomic data, the Government carried on with efforts by seeking IMF technical assistance for the implementation of the IMF's new 2017 quarterly national accounts handbook. Work began in the first quarter and a mission to finalize this work is expected by the end of the year.

101. In addition, the base year of the Harmonized Consumer Prices Index (IHPC) has been revised from 2008 to 2014. Revision is underway of the retrospective series which should be available by September 2019. 
102. In order to improve macroeconomic forecasts, an IPA diagnostic mission was conducted during the first half of 2018 by external consultants, thanks to European Union support. At the end of the mission, a roadmap was drawn up with actions to be implemented for updating and improving IPA by the end of 2019. Such improvements will include the adoption of the INSD National Accounting (SCN 2008) ERR model, the rewriting of the TOFE modules, Balance of Payments and Monetary Situation according to the new IMF manuals.

\section{Governance}

103. The ASCE-LC is under restructuring since the adoption of Law No. 004-2015/CNT of March 2015 on the fight against corruption with the creation of five departments: the audit department, the corruption prevention department, the enquiries and investigation department, the litigation follow-up department and the department in charge of digitalization of declarations of interest and assets. As a result, it now has a guidance council composed of nine councillors. It should also be noted that the position of Controller General is now filled by call for applications for a nonrenewable 5-years term.

104. As part of the fight against corruption, regularl consultations are held with the other control bodies. In addition, actions are being taken with social partners to revitalize disciplinary boards. Furthermore, a fact-finding unit was set up to investigate ill-gotten property. For this purpose, study visits are planned in Cameroon and Tunisia.

105. The institution of an annual management audit of the Government has been effective since 2016. As such, management audit reports with recommendations for 2016 and 2017 are available and published on the ASCE-LC website. The 2018 report is being drafted. To monitor the implementation of the audit recommendations, two sessions are planned per year. To that effect, a first release recorded a $51 \%$ implementation of the recommendations. Another release is planned for the month of June 2019 in addition to the first that could not cover all ministries.

106. In perspective, a symposium is planned for late October - early November for a synergy of action with the other control bodies (Technical Inspectorate of Services of Sector Ministries) and the judiciary.

107. The government remains committed to address the risks related to anti-money laundering and terrorism financing (AML/CFT). Building on the WAEMU AML/CFT directive approved in 2015, Burkina Faso authorities adopted in 2016 their national AML/CFT directive through the Law 0162016/AN of May 03 2016. Surveillance of AML/CFT issues is mostly coordinated by CENTIF whose supervisory unit comprises representatives from the Ministry of Finance, Ministry of Security, and members of the judiciary and the BCEAO. An evaluation report assessing the AML/CFT situation in 2017 was produced by CENTIF. The report shows a 37 percent increase in the number of suspect bank transactions relative to 2016, however the increase essentially reflects better reporting from banks in the context of increased awareness campaign conducted by CENTIF. While the existing framework is adequate and provides CENTIF with the necessary legal tools to address AML/CFT risks, the lack of staff, resources and insufficient international cooperation have limited CENTIF work 
to merely administrative activities. Investigation of suspect transactions is therefore an area that is lagging behind and the authorities are committed to step up efforts to reverse the situation. The CENTIF is working on the 2018 report which is expected to discuss the AML/CFT weaknesses in Burkina Faso and propose recommendations. This report will be made public.

\section{J. Program Monitoring}

108. Government will take all necessary measures to meet the targets and criteria as set out in Tables 1 and 3 of this memorandum. The program will be monitored via semi-annual reviews based on the PCs set through end-December 2019 and ITs set through end-June 2020 as described in Table 3 and the structural conditionality as described in Table 4. The third and fourth reviews are expected to be completed on or after December 15, 2019 and June 15, 2020, respectively. 
Table 1. Burkina Faso: Quantitative Performance Criteria and Indicative Targets for ECF Arrangement, 2018 (CFAF billions)

\begin{tabular}{|c|c|c|c|c|c|c|}
\hline & \multicolumn{6}{|c|}{2018} \\
\hline & \multicolumn{2}{|c|}{ Sept. 1/ } & \multicolumn{4}{|c|}{ Dec. } \\
\hline & Prog. & Outturn & Prog. & Adj. & Outturn & Status \\
\hline \multicolumn{7}{|l|}{ Quantitative Performance Criteria } \\
\hline Ceiling on net domestic financing of the government $2 / 3 / 4 / 5 /$ & 258 & 239 & 258 & 380 & 395 & Not met \\
\hline Ceiling on the amount of nonconcessional external debt contracted or guaranteed by the government $3 / 6$ / & 200 & 52 & 200 & 200 & 56 & Met \\
\hline Ceiling on the accumulation of external payment arrears by the government $7 /$ & 0 & 0 & 0 & 0 & 0 & Met \\
\hline Ceiling on the guaranteeing of new domestic loans to suppliers and contractors by the government $7 /$ & 0 & 0 & 0 & 0 & 0 & Met \\
\hline Ceiling on government guarantees on new bank pre-financing for public investments $7 /$ & 0 & 0 & 0 & 0 & 0 & Met \\
\hline \multicolumn{7}{|l|}{ Indicative Targets } \\
\hline Ceiling on the accumulation of domestic arrears by the government $7 /$ & 0 & 0 & 0 & 0 & 0 & Met \\
\hline Ceiling on the overall fiscal deficit including grants $2 / 3$ / & 312 & 212 & 382 & 436 & 382 & Met \\
\hline Floor on government revenue $3 /$ & 1,011 & 1,105 & 1,444 & 1,444 & 1,531 & Met \\
\hline Floor on poverty-reducing current social expenditures $3 /$ & 132 & 188 & 176 & 176 & 204 & Met \\
\hline Ceiling on the stock of certified and unpaid VAT refunds older than 30 days $3 /$ & 55 & 41 & 55 & 55 & 27 & Met \\
\hline Ceiling on the value of PPPs contracted $3 /$ & 200 & 0 & 200 & 200 & 0 & Met \\
\hline \multicolumn{7}{|l|}{ Memorandum Item } \\
\hline Ceiling on the amount of concessional external debt contracted or guaranteed by the government 3 / & 550 & 414 & 574 & 574 & 486 & Met \\
\hline
\end{tabular}

Sources: Burkinabè authorities; and IMF staff estimates and projections.

1/ Indicative Target, except for continuous performance criteria.

2/ The net domestic financing ceiling is adjusted to reflect the shortfall in program grants and loans, while the overall deficit is adjusted to reflect the shortfall in program grants.

3/ Cumulative from January 1, 2018.

4/ The ceiling on net domestic financing will be lowered by the full proceeds of any private concessional budget support loan as specified in the TMU.

5/ The ceiling on net domestic financing will be adjusted upward by the amount of securitization of SONABHY losses owing to below cost-recovery sales of petroleum products in 2018 and capped to a maximum of CFAF 80 billion

6/ The limit is not tied to specific projects.

7/ To be observed continuously. 


\begin{tabular}{|c|c|c|c|}
\hline Benchmark & Objective & Completion Date & Status \\
\hline $\begin{array}{l}\text { Establish a limit on the amount of PPPs } \\
\text { that can be contracted by the Government }\end{array}$ & $\begin{array}{l}\text { Improve budget } \\
\text { transparency and mitigate } \\
\text { fiscal risks }\end{array}$ & April 2018 & $\begin{array}{l}\text { Not met (met } \\
\text { with delay) }\end{array}$ \\
\hline $\begin{array}{l}\text { Include as an Annex to the } 2019 \text { Budget } \\
\text { law an analysis of the costs-benefits of the } \\
\text { ten largest investment projects, including } \\
\text { PPP projects }\end{array}$ & $\begin{array}{l}\text { Improve budget } \\
\text { transparency and mitigate } \\
\text { fiscal risks }\end{array}$ & December 2018 & Met \\
\hline $\begin{array}{l}\text { Develop and adopt a framework for } \\
\text { transitioning to a single treasury account }\end{array}$ & $\begin{array}{l}\text { Improve treasury } \\
\text { management }\end{array}$ & December 2018 & Not met \\
\hline $\begin{array}{l}\text { Integrate the results of the artisanal gold } \\
\text { study into the system of national accounts } \\
\text { and revise the base year }\end{array}$ & $\begin{array}{l}\text { Improve the accuracy of } \\
\text { national statistics }\end{array}$ & December 2018 & Met \\
\hline $\begin{array}{l}\text { Complete the revision of national accounts } \\
\text { statistics to the new base year, and } \\
\text { disseminate the revised series }\end{array}$ & $\begin{array}{l}\text { Improve the accuracy of } \\
\text { national statistics }\end{array}$ & December 2018 & Not met \\
\hline $\begin{array}{l}\text { Proceed with the dematerialization of asset } \\
\text { declarations of government officials and } \\
\text { those covered by the law, by instituting } \\
\text { online submissions, and create a } \\
\text { searchable database }\end{array}$ & $\begin{array}{l}\text { Improve governance and } \\
\text { improve the fight against } \\
\text { corruption }\end{array}$ & December 2018 & Not met \\
\hline $\begin{array}{l}\text { Raise the rate of tax on non-alcoholic } \\
\text { beverages from } 10 \text { percent to } 15 \text { percent }\end{array}$ & Increase revenue & January 2019 & Met \\
\hline $\begin{array}{l}\text { Increase tax on airline tickets from CFAF } \\
1000 \text { to CFAF } 20000 \text { for economy class and } \\
\text { from CFAF } 7000 \text { to CFAF } 40000 \text { for } \\
\text { business class }\end{array}$ & Increase revenue & January 2019 & Met \\
\hline $\begin{array}{l}\text { Eliminate the flat-rate tax system for } \\
\text { beverage sector businesses and place them } \\
\text { under the standard tax regime by } \\
\text { introducing a } 5 \text { percent tax as an advance } \\
\text { income tax payment }\end{array}$ & Increase revenue & January 2019 & Met \\
\hline $\begin{array}{l}\text { Publish the implicit subsidy of fuel pump } \\
\text { prices on the website of the Ministry of } \\
\text { Economy, Finance and Development on a } \\
\text { monthly basis }\end{array}$ & $\begin{array}{l}\text { Increase transparency on } \\
\text { the fiscal cost of fuel } \\
\text { subsidies }\end{array}$ & January 2019 & Met \\
\hline $\begin{array}{l}\text { Make the inspection certificate compulsory } \\
\text { for checking transactions for customs } \\
\text { clearance purposes and ensure documents } \\
\text { are scanned in ASYCUDA World }\end{array}$ & $\begin{array}{l}\text { Increase revenue and } \\
\text { protect the revenue base }\end{array}$ & April 2019 & Met \\
\hline
\end{tabular}




\begin{tabular}{|c|c|c|c|}
\hline \multicolumn{4}{|c|}{ Table 2. Burkina Faso: Structural Benchmarks $-2^{\text {nd }}$ Review (concluded) } \\
\hline $\begin{array}{l}\text { Publish the number of public } \\
\text { servants on the government } \\
\text { payroll according to grade, } \\
\text { region and ministry }\end{array}$ & $\begin{array}{l}\text { Contain public-sector } \\
\text { wage bill }\end{array}$ & April 2019 & Not met \\
\hline $\begin{array}{l}\text { Approval by the Cabinet of } \\
\text { Ministers of the } 2020-2022 \\
\text { Multiannual Budget and } \\
\text { Economic Programming } \\
\text { Document with a continuous } \\
\text { reduction in the ratio of the } \\
\text { wage bill to tax revenue ratio } \\
\text { from its peak of } 55 \text { percent } \\
\text { in } 2019\end{array}$ & $\begin{array}{l}\text { Contain public-sector } \\
\text { wage bill }\end{array}$ & Mid-June 2019 & Met \\
\hline
\end{tabular}


Table 3. Burkina Faso: Quantitative Performance Criteria and Indicative Targets for ECF Arrangement, 2019-20 (CFAF billions)

\begin{tabular}{|c|c|c|c|c|c|c|c|}
\hline & \multicolumn{5}{|c|}{2019} & \multicolumn{2}{|c|}{2020} \\
\hline & \multicolumn{2}{|c|}{ Mar. 1/ } & June & Sept. 1/ & Dec. & Mar. 1/ & June $1 /$ \\
\hline & Prog. & Prel. & \multicolumn{3}{|c|}{ Prog. } & \multicolumn{2}{|c|}{ Prog. } \\
\hline \multicolumn{8}{|l|}{ Quantitative Performance Criteria } \\
\hline Ceiling on net domestic financing of the government $2 / 3 / 4 / 5$ / & 0 & 70 & 100 & 146 & 200 & 60 & 62 \\
\hline Ceiling on the amount of external debt contracted or guaranteed by the government (PV) $2 / 6 / 7 /$ & 370 & 6 & 370 & 370 & 370 & 410 & 410 \\
\hline Ceiling on the accumulation of external payment arrears by the government 8 / & 0 & 0 & 0 & 0 & 0 & 0 & 0 \\
\hline Ceiling on the guaranteeing of new domestic loans to suppliers and contractors by the government 8/ & 0 & 0 & 0 & 0 & 0 & 0 & 0 \\
\hline Ceiling on government guarantees of new bank pre-financing for public investments 8 / & 0 & 0 & 0 & 0 & 0 & 0 & 0 \\
\hline \multicolumn{8}{|l|}{ Indicative Targets } \\
\hline Ceiling on the accumulation of domestic arrears by the government 8 / & 0 & 0 & 0 & 0 & 0 & 0 & 0 \\
\hline Ceiling on the overall fiscal deficit including grants $2 / 3 /$ & 0 & 9 & 130 & 196 & 258 & 0 & 141 \\
\hline Floor on government revenue $2 /$ & 376 & 396 & 798 & 1262 & 1697 & 415 & 880 \\
\hline Floor on poverty-reducing current social expenditures $2 /$ & 48 & 73 & 95 & 143 & 190 & 49 & 98 \\
\hline Ceiling on the stock of certified and unpaid VAT refunds older than 30 days $2 /$ & 45 & 44 & 40 & 40 & 40 & 40 & 40 \\
\hline Ceiling on the value of PPPs contracted $2 /$ & 200 & 0 & 200 & 200 & 200 & 200 & 200 \\
\hline
\end{tabular}

Sources: Burkinabè authorities; and IMF staff estimates and projections.

$1 /$ Indicative target, except for continuous performance criteria.

2/ Cumulative from January 1, 2019.

$3 /$ The ceiling on net domestic financing will be adjusted to reflect the shortfall in program grants and loans, while the overall deficit will be adjusted to reflect the shortfall in program grants.

4/ The ceiling on net domestic financing will be adjusted upward by the amount of securitization of SONABHY losses owing to below cost-recovery sales of petroleum products by end-June 2018, up to a maximum of CFAF

97 billion, as specified in the TMU.

5/ The ceiling on net domestic financing will be lowered by the full proceeds of any concessional budget support loan used for debt management purposes as specified in the TMU.

6/ The limit is not tied to specific projects.

7/ The ceiling on the amount of external debt will be increased up by the present value of the full amount of any concessional budget support loan used for debt management purposes as specified in the TMU.

8/ To be observed continuously. 


\begin{tabular}{|c|c|c|}
\hline & & \\
\hline \multirow{2}{*}{\multicolumn{3}{|c|}{ Prior Actions }} \\
\hline & & \\
\hline $\begin{array}{l}\text { Public statement of the government on its } \\
\text { commitment and strategy to gradually reduce the } \\
\text { ratio of the wage bill to tax revenue starting in } \\
2020\end{array}$ & Contain public-sector wage bill & Prior action \\
\hline $\begin{array}{l}\text { Publish the number of public servants on the } \\
\text { government payroll according to grade, region } \\
\text { and ministry }\end{array}$ & Contain public-sector wage bill & Prior action \\
\hline \multicolumn{3}{|c|}{ Structural benchmarks } \\
\hline $\begin{array}{l}\text { Establish a special account for VAT credit refunds } \\
\text { that is funded with a portion of VAT revenues and } \\
\text { produce quarterly reports on the operational } \\
\text { management of this account }\end{array}$ & $\begin{array}{l}\text { Strengthen the integrity of the } \\
\text { VAT system }\end{array}$ & June 2019 \\
\hline $\begin{array}{l}\text { Appoint an independent external auditor to } \\
\text { conduct an audit of SONABHY's operations, } \\
\text { financial position, and accounting practices. }\end{array}$ & $\begin{array}{l}\text { Reduce fiscal risks and eliminate } \\
\text { fiscal costs associated with } \\
\text { subsidies }\end{array}$ & $\begin{array}{l}\text { July } 2019 \\
\text { (reset from April 2018) }\end{array}$ \\
\hline $\begin{array}{l}\text { Complete the revision of national accounts } \\
\text { statistics to the new base year and disseminate } \\
\text { the revised series. }\end{array}$ & $\begin{array}{l}\text { Improve the accuracy of national } \\
\text { statistics. }\end{array}$ & $\begin{array}{l}\text { September } 2019 \\
\text { (reset from December } \\
\text { 2018) }\end{array}$ \\
\hline Operationalize the Treasury Single Account & Improve treasury management & $\begin{array}{l}\text { September } 2019 \\
\text { (reset from December } \\
\text { 2018) }\end{array}$ \\
\hline $\begin{array}{l}\text { Conduct a study on the taxation system of small- } \\
\text { and medium-sized enterprises }\end{array}$ & Strengthen tax administration & September 2019 \\
\hline $\begin{array}{l}\text { Reduce the number of new civil service staff } \\
\text { members in } 2019 \text { compared to the initial } \\
\text { projection of } 10,615 \text { hires (modified). }\end{array}$ & Tighten control of the wage bill & September 2019 \\
\hline $\begin{array}{l}\text { In the appropriate budget accounts, budget and } \\
\text { account for revenue from/expenditure on prime } \\
\text { de motivation in the } 2020 \text { budget law (modified). }\end{array}$ & Improve fiscal transparency & September 2019 \\
\hline $\begin{array}{l}\text { Submit to the National Assembly a } 2020 \text { budget } \\
\text { proposal that is fully aligned with the projected } \\
\text { wage bill of the ECF-supported program (new). }\end{array}$ & Contain public-sector wage bill & September 2019 \\
\hline $\begin{array}{l}\text { Harmonize the taxation of tobacco in line with the } \\
\text { new WAEMU directive by setting the rate at a } \\
\text { minimum of } 50 \text { percent }\end{array}$ & Increase revenue & November 2019 \\
\hline Include PPPs in the public investment program & Mitigate fiscal risks & December 2019 \\
\hline $\begin{array}{l}\text { Proceed with the dematerialization of asset } \\
\text { declarations of government officials and those } \\
\text { covered by the law, by instituting online } \\
\text { submissions, and create a searchable database }\end{array}$ & $\begin{array}{l}\text { Improve governance and } \\
\text { improve the fight against } \\
\text { corruption }\end{array}$ & $\begin{array}{l}\text { March } 2020 \\
\text { (reset from December } \\
\text { 2018) }\end{array}$ \\
\hline
\end{tabular}




\section{Attachment 2. Technical Memorandum of Understanding}

1. This Technical Memorandum of Understanding (TMU) defines the quantitative performance criteria, indicative targets, and structural benchmarks that will serve to assess performance under the program supported by the Extended Credit Facility (ECF). It also sets the framework and deadlines for the submission of data to IMF staff for assessment of program implementation.

\section{CONDITIONALITY}

2. The quantitative performance criteria and indicative targets are provided in Table 1 of the MEFP. The structural benchmarks set forth in the program are presented in Tables 2 and 4 of the MEFP.

\section{DEFINITIONS}

3. Government. Unless otherwise indicated, the term "government" means the central government of Burkina Faso and does not include local governments, the central bank, or any other public or government-owned entity with autonomous legal status not included in the table detailing the financial operations of the state (TOFE).

4. Definition of debt. The definition of debt is set out in IMF Executive Board Decision No. 15688-(14/107), Point 8, as published on the IMF website. The term "debt" will be understood to mean all current, i.e. not contingent, liabilities, created under a contractual arrangement through the provision of value in the form of assets (including currency) or services, and which requires the obligor to make one or more payments in the form of assets (including currency) or services, at some future point(s) in time; these payments will discharge the principal and/or interest liabilities incurred under the contract. Debts can take various forms; the primary ones being as follows:

(i) loans, i.e., advances of money to the obligor by the lender made on the basis of an undertaking that the obligor will repay the funds in the future (including deposits, bonds, debentures, commercial loans and buyers' credits) and temporary exchanges of assets that are equivalent to fully collateralized loans under which the obligor is required to repay the funds, and usually pay interest, by repurchasing the collateral from the buyer in the future (such as repurchase agreements and official swap arrangements);

(ii) suppliers' credits, i.e., contracts where the supplier permits the obligor to defer payments after the date on which the goods are delivered or services are provided; and

(iii) leases, i.e., arrangements under which property is provided which the lessee has the right to use for one or more specified period(s) of time that are usually shorter than the total expected service life of the property, while the lessor retains the title to the property. For the purpose of these guidelines, the debt is the present value (at the inception of the lease) of all lease payments expected to be made during the period of the agreement excluding those payments that cover the operation, repair, or maintenance of the property. 
5. Under the definition of debt set out above, arrears, penalties, and judicially awarded damages arising from the failure to make payment under a contractual obligation that constitutes debt are debt. Failure to make payment on a contractual obligation that is not considered debt under this definition (e.g., payment on delivery) will not give rise to debt.

6. Debt guarantees. A government debt guarantee means an explicit legal obligation to service a debt in the event of nonpayment by the borrower (through payment in cash or in kind).

7. Debt concessionality. A debt is considered concessional if it includes a grant element of at least 35 percent. $^{1}$ The present value (PV) of debt at the time it is contracted is calculated by discounting the borrower's future debt service payments on the debt. ${ }^{2}$ The discount rates used is 5 percent.

8. External debt. External debt is defined as debt contracted or serviced in a currency other than the CFA franc. The relevant performance criteria apply to the external debt of the government, public enterprises that receive government transfers, and other public entities in which the government holds more than 50 percent of the capital, and any private debt for which the government has extended guarantees that constitute a contingent liability for the government.

\section{QUANTITATIVE PERFORMANCE CRITERIA}

9. The quantitative performance criteria for 2019 and 2020 are as follows:

(i) a ceiling on net domestic financing of the government;

(ii) a ceiling on the contracting or guaranteeing of external debt by the government in PV terms;

(iii) a ceiling on the accumulation of payment arrears on external debt service;

(iv) a ceiling on the guaranteeing of domestic loans to suppliers and contractors;

(v) a ceiling on the guaranteeing of bank pre-financing of public investments.

\section{A. Net Domestic Financing of the Government}

10. Net domestic financing is defined as the sum of (i) net bank credit to the government, including net bank credit to the government as defined below vis-à-vis the national banking institutions (claims associated with IMF disbursements are included); (ii) the stock of unredeemed government bills and bonds held outside national commercial banks; (iii) privatization receipts and other government claims and debts vis-à-vis national nonbank institutions. Net bank credit to the

\footnotetext{
${ }^{1}$ This IMF webpage provides a tool to compute the grant element in a large range of financial arrangements: http://www.imf.org/external/np/pdr/conc/calculator

2 The calculation of the concessionality takes into account all aspects of the debt contract, including the date of payment, the grace period, the schedule, the commissions and management fees.
} 
government is the balance of the government's claims and debts vis-à-vis national banking institutions. Government claims include the cash holdings of the Burkinabè government, deposits with the central bank, deposits with commercial banks, and secured obligations, and government deposits in postal checking accounts (CCP). Government debt to the banking system includes funding from the central bank (including statutory advances, consolidated advances, IMF financing, and refinancing of secured obligations), government securities held by the central bank, and funding from commercial banks (including government securities held by commercial banks). Net bank credit to the government is calculated based on information provided in the TOFE. The foregoing items are calculated based on the government budget execution report presented each month in the government flow-of-funds table prepared by the Ministry of the Economy and Finance.

\section{Adjustment}

11. The cumulative ceiling on net domestic financing from the beginning of any calendar year will be adjusted upward in the amount by which external program support to central government falls short of the amount projected, in the event the external program assistance is lower than programmed, up to a maximum of CFAF 80 billion. The shortfall will be calculated in relation to the projections in Table 1 below. The ceiling will not be adjusted downward in the event the external program assistance is higher than programmed.

\begin{tabular}{|c|c|c|}
\hline \multicolumn{3}{|c|}{$\begin{array}{l}\text { Table 1. Projected External Program Assistance } \\
\text { (Cumulative, CFAF Billions) }\end{array}$} \\
\hline & End-June 2019 & End-December 2019 \\
\hline Program grants and loans & 73 & 113 \\
\hline (Of which program grants) & (55) & (93) \\
\hline
\end{tabular}

12. The cumulative ceiling on net domestic financing will be adjusted downward by the full amount of any disbursement of concessional budget support loans related to debt management operations aiming at replacing the relatively more expensive domestic debt.

13. The ceiling on net domestic financing will be adjusted upward by the amount of securitization of accumulated SONABHY losses owing to below cost recovery sales of petroleum products by end-June 2018, up to a maximum of CFAF 97 billion. This adjustor will only apply to the securitization proceeds from 2018 which have not yet been cashed by SONABHY.

14. The Ministry of the Economy and Finance will forward data on net domestic financing to the IMF within six weeks after the end of each quarter. 


\section{B. New Non-Concessional External Debt Contracted or Guaranteed by the Government and Present Value of External Debt Contracted or Guaranteed by the Government}

15. This performance criterion applies to external debt as defined in paragraph 8 of this memorandum. It utilizes the concept of concessionality as defined in paragraph 7 of this memorandum. This performance criterion also applies to any private debt guaranteed by the government that constitutes a contingent government debt as defined in paragraphs 4 to 7 of this memorandum. For the purpose of this performance criterion, "government" shall include the central government of Burkina Faso, public enterprises that receive government transfers, local governments, and other public-sector entities (including public administrative, professional, scientific and technical agencies). However, this performance criterion will not apply to government bills and bonds issued in CFA francs on the WAEMU regional market, to suppliers' normal shortterm credits, or to IMF loans. It is measured on a cumulative basis from the date of the IMF Executive Board's approval of the ECF arrangement, and no adjustment factor will apply.

16. For 2019, the government undertakes not to contract or guarantee external debt (concessional or non-concessional) with a total PV in excess of the ceiling indicated in MEFP table 3, measured on a cumulative basis from the start of the year (i.e. January 1,2019 ). In case of a concessional budget support loan referred to in the paragraph 12, the ceiling will be raised by the $\mathrm{PV}$ of the full amount of the loan. All other provisions and definitions in paragraph 12 will continue to apply.

17. The proceeds of any concessional budget support loan referred to in paragraphs 12 and 19 will be used only to contribute to the financing of the gross financing requirements of the government that are consistent with the overall fiscal deficit ceilings of 3 percent of GDP. Any surplus of resources will be saved.

\section{Reporting Deadlines}

18. Details on any loan (terms and creditors) contracted by the government must be reported within four weeks of the end of each month. The same requirement applies to guarantees extended by the government.

\section{Non-accumulation of New External Payment Arrears by the Government}

19. External payment arrears are external payments due but unpaid. Under the program the government agrees not to accumulate external payment arrears on its debt, except arrears arising from external payment obligations being renegotiated with creditors, including bilateral non-Paris Club creditors. Non-accumulation of new external arrears by the government is a performance criterion to be observed continuously. 


\section{Guaranteeing of New Domestic Loans to Suppliers and Contractors by the Government}

20. The government undertakes to not provide new financial guarantees for domestic loans to its suppliers or contractors. This performance criterion shall be observed continuously. For this performance criterion, "government" includes the central government, institutions of an industrial or commercial nature (EPIC), public administrative agencies (EPA), public scientific and technical institutes, public vocational establishments, public health agencies, local authorities, public enterprises, national corporations, semi-public corporations (public corporations with financial autonomy, in which the government holds at least 50 percent of the capital), and state agencies.

\section{E. Guaranteeing of New Bank Pre-Financing for Public Investments by the Government}

21. The government undertakes not to guarantee new bank pre-financing for public investments. In a pre-financing arrangement, a private company granted a public works contract by the government obtains a loan from a domestic commercial bank or a group of commercial banks. The Ministry of Finance guarantees this loan and, at the same time, signs an unconditional and irrevocable substitution of debtor agreement to service all principle and interest. For this criterion, government includes the central government, institutions of an industrial or commercial nature (EPIC), public administrative agencies (EPA), public scientific and technical institutes, public vocational establishments, public health agencies, local authorities, public enterprises, national corporations, semi-public corporations (public corporations with financial autonomy, in which the government holds at least 50 percent of the capital), and state agencies. This performance criterion shall be observed continuously.

\section{QUANTITATIVE INDICATIVE TARGETS}

22. The program also includes quantitative indicative targets for:

(i) the accumulation of domestic arrears;

(ii) the overall fiscal deficit (commitment basis, grants included);

(iii) total government revenue;

(iv) poverty-reducing current social expenditures;

(v) value added tax refunds; and

(vi) public-private partnerships. 


\section{A. Accumulation of Domestic Arrears by the Government}

23. The government will not accumulate payment arrears on domestic obligations during the program period. For this indicative target, a "domestic obligation" is one serviced in CFA francs, but it excludes government liabilities to local governments or any other public or government-owned entity with autonomous legal status not included in the table detailing the financial operations of the state (TOFE), except the central bank. Except in cases where the terms and conditions of the transaction stipulate a longer period, payments are deemed to be in arrears in keeping with the following definition:

(i) Debt unpaid for more than 30 days after the due date stipulated in the agreement between the parties (creditor/debtor).

(ii) Wages or pensions unpaid 90 days after their due date.

(iii) Payments for goods and services rendered received more than 90 days after processing of the supporting documents submitted by suppliers.

\section{B. Overall Fiscal Deficit Including Grants}

\section{Definition}

24. For the program, the overall deficit including grants is valued on a commitment basis. It is defined as the sum of the government's net foreign and domestic financing, measured from the financing side, plus a cash basis adjustment. Net foreign financing is the sum of new foreign borrowing less amortization. Government net domestic financing is defined in paragraphs 10 and 11 above. The cash basis adjustment is defined as the sum of: (i) the total change in unauthorized expenditure commitments, (ii) the change in pending bills, and (iii) the change in government deposits.

\section{Adjustment}

25. The ceiling on the overall fiscal deficit will be adjusted upward in the amount by which actual external program grants to central government falls short of the amount projected, up to a maximum of CFAF 80 billion. The shortfall will be calculated in relation to the projections in Table 1 above. The ceiling will not be adjusted downward should actual external program grant assistance be higher than projected.

\section{Government Revenue}

\section{Definition}

26. Government revenue is valued on a cash basis. It includes all tax and non-tax revenue collected by the Directorate General of Taxation, the Directorate General of Customs, the Burkinabè 
Treasury, and other government revenue collection units. It also includes revenue from treasury checks.

\section{Poverty-Reducing Current Social Expenditures}

\section{Definition}

27. Social spending is the sum of current expenditure included in the social spending program as defined in the budget. This social spending program is defined as the sum of budget programs or parts of programs that target poor households and: (i) ensure access to basic social services;

(ii) promote access to health services and nutrition programs; (iii) fight against HIV/AIDS;

(iv) promote access to drinkable water; (v) improve living conditions, including environment and sanitation; or (vi) ensure social protection. Within these programs or parts of programs, only budget lines classified as social spending are retained.

\section{E. Certified and Unpaid VAT Refunds Older than 30 Days}

\section{Definition}

28. For the program, the stock of value added tax (VAT) refund claims that have been certified but remain unpaid for more than 30 days is comprised of signed tax refund amounts. The 30-day period starts from the date of signature of the tax refund certificate by the Director General of Taxes.

\section{F. Public Private Partnerships}

\section{Definition}

29. A public-private partnership is defined as a long-term contract between a private party and a government entity, for providing a public asset or service, in which the private party bears significant risk and management responsibility, and remuneration is linked to performance. It excludes pre-financing and supplier credit arrangements.

\section{Additional Information for Program Monitoring}

30. To enable IMF staff to assess program performance, the government agrees to submit the following data to them, in paper format and/or MS Excel electronic files, with the frequencies and deadlines specified below. 
Table 2. Summary of Data Reporting Requirements

\section{Information}

Institution

Data

Responsible Frequency

Reporting

Frequency

\section{Public Finance}

The government's fiscal reporting table (TOFE) and the customary appendix tables; (if data on actual investment

Ministry of

Monthly

6 weeks

financed by external grants and loans are not available in time, a

Economy,

linear estimate of execution based on the annual projections will

be used)

Finance and

Development

(MINEFID)

Domestic budgetary financing (net bank credit to the

MINEFID/

Monthly

6 weeks

government and stock of unredeemed government bonds and

BCEAO bills)

Monthly data on the execution rates by the customs office relative to monthly forecasts.

MINEFID/

DGD

Monthly 6 weeks

A quarterly report on the outcomes and actions undertaken to

DGD/DGI

Quarterly

6 weeks

put in place a better control and supervision of taxpayers using

the single taxpayer identification number to cross-check

information between DGI and DGD, starting with large taxpayers.

Data on implementation of the public investment program,

DGB/DGTCP Quarterly 6 weeks

including details on financing sources

The stock of external debt, external debt service, external debt contracted, and external debt repayment

DGTCP

Quarterly

6 weeks

Social poverty-reducing expenditures in table format

DGTCP

Monthly

6 weeks

Petroleum product prices, consumption and taxes, including:

(i) the price structure for the month concerned; (ii) detailed

SONABHY/

Monthly

4 weeks

calculation of the price structure, from the fob-MED price to the

retail price; (iii) volumes purchased and distributed for

consumption by the petroleum distributor (SONABHY); with a

distinction made between retail and industry sales; and (iv) a

breakdown of tax revenue from petroleum products - customs

duties, tax on petroleum products (TPP) and value-added tax

(VAT) - and subsidies unpaid

DGTCP

A quarterly summary report of monthly data of SONABHY's

accounts including gains and/or losses from the buying and

SONABHY/

Quarterly

6 weeks

selling of hydrocarbon products by type of product, cash flows

position and income statement, taking into account all received

MINEFID

subsidies and government securities issued or sold in the

banking system or else.

A quarterly summary report of monthly data of SONABEL's

accounts including its cash flows position and income statement

SONABEL/

Quarterly

6 weeks

and taking into account all received subsidies and project grants

MINEFID

and loans from the technical and financial partners. 
Table 2. Summary of Data Reporting Requirements (continued)

A monthly statement of the accounts with the treasury, broken DGTCP

Monthly

6 weeks

out by major category (administrative services, state enterprises,

joint public-private enterprises, public administrative enterprises,

international organizations, private depositors, and others)

\begin{tabular}{llll}
\hline A quarterly activity report from the Investigation and Intelligence & DGI/DGD & Quarterly & 6 weeks \\
Directorate including taxpayer controls across DGI and DGD & & \\
using the unique taxpayer identification number, beginning with & & \\
large taxpayers. & & & \\
\hline
\end{tabular}

Provide monthly customs revenue projections (on an annualized $\quad$ DGD $\quad$ Monthly 6 weeks

basis) by customs post, and report on monthly outcomes

compared to projections.

Keep 'Champ 44' enabled for input of references from inspection DGD Continuous

notices for all customs declarations.

Provide monthly DGI revenue projections (on an annualized $\quad$ DGI $\quad$ Monthly 6 months

basis) by type, and report on monthly outcomes compared to

projections.

Provide monthly DGTCP revenue projections (on an annualized

basis) by type, and report on monthly outcomes compared to

projections.

\begin{tabular}{|c|c|c|c|}
\hline $\begin{array}{l}\text { A quarterly summary report of VAT refunds, including transfers } \\
\text { received from the ACCT, cumulative amount paid since the } \\
\text { beginning of the year, the stock of certified refund claims } \\
\text { (Régisseur d'avance), and total VAT refund claims being } \\
\text { processed (DGE, DLC) }\end{array}$ & DGI & Quarterly & 3 months \\
\hline $\begin{array}{l}\text { A monthly update of the PPP and sovereign guarantee } \\
\text { databases }\end{array}$ & $\begin{array}{l}\text { DGCOOP/ } \\
\text { MINEFID }\end{array}$ & & 4 weeks \\
\hline Monthly estimate of the implicit pump fuel price subsidy & $\mathrm{CIDPH}$ & & 4 weeks \\
\hline \multicolumn{4}{|l|}{ The consolidated balance sheet of monetary institutions } \\
\hline The consolidated balance sheet of monetary institutions & $\begin{array}{l}\text { NDs of the } \\
\text { BCEAO }\end{array}$ & Monthly & 6 weeks \\
\hline The monetary survey: provisional data & BCEAO & Monthly & 6 weeks \\
\hline The monetary survey: final data & BCEAO & Monthly & 10 weeks \\
\hline The lending and borrowing interest rates & BCEAO & Monthly & 6 weeks \\
\hline $\begin{array}{l}\text { The standard bank supervision indicators for banks and nonbank } \\
\text { financial institutions }\end{array}$ & BCEAO & Monthly & 6 weeks \\
\hline \multicolumn{4}{|l|}{ Balance of Payments } \\
\hline Preliminary annual balance of payments data & BCEAO & Annual & 9 months \\
\hline Foreign trade statistics & $\begin{array}{l}\text { INSD/ } \\
\text { MINEFID }\end{array}$ & Monthly & 3 months \\
\hline $\begin{array}{l}\text { Any revision of balance of payments data (including services, } \\
\text { private transfers, official transfers, and capital transactions) }\end{array}$ & BCEAO & $\begin{array}{l}\text { As they } \\
\text { occur }\end{array}$ & 2 weeks \\
\hline
\end{tabular}


Table 2. Summary of Data Reporting Requirements (concluded)

\section{Real Sector}

Provisional national accounts; and any revision of the national accounts

Disaggregated monthly consumer price indices

MINEFID Annual 2 weeks

\section{Structural Reforms and Other Data}

Any study or official report on Burkina Faso's economy, on the

MINEFID

2 weeks date published, or the date of entry into force.

Any decision, order, law, decree, ordinance, or circular having

MINEFID

2 weeks economic or financial implications, on the date published, or the date of entry into force. 


\title{
INTERNATIONAL MONETARY FUND
}

\section{BURKINA FASO}

SECOND REVIEW UNDER THE EXTENDED CREDIT FACILITY

July 17, 2019

\author{
ARRANGEMENT, REQUEST FOR WAIVERS OF \\ NONOBSERVANCE OF A PERFORMANCE CRITERION, DE \\ MINIMIS MISREPORTING RESULTING IN NONCOMPLYING \\ DISBURSEMENT-SUPPLEMENTARY INFORMATION AND \\ SUPPLEMENTARY LETTER OF INTENT
}

\author{
Approved By \\ Dominique Desruelle \\ Prepared by the African Department in consultation with SPR \\ (AFR) and Edward \\ Gemayel (SPR) \\ and LEG
}

This supplement provides an update on Burkina Faso's external arrears since the staff report for the second review under the ECF arrangement was issued. New information received by staff indicates nonobservance of the continuous performance criteria $(P C)$ on accumulation of external arrears. However, considering that the arrears have been now repaid and the nature of the non-observance has been minor, temporary and appropriate corrective actions were taken, staff supports the authorities' request for a waiver for non-observance of the PC. The additional information does not change the thrust of the staff appraisal and staff continues to recommend the completion of the second review.

\section{Staff received on July 11,2019 , additional information on minor external} arrears that were temporarily accumulated to one official creditor. These arrears are related to payments due to the Spanish government and amount to EUR 494,383 as of end-June 2019. The Burkinabe authorities became aware of the arrears only recently. These arrears are due to a legacy lapse in debt recording. The loan was contracted in 2008 and had a grace period of nine years starting when the loan became effective. When the loan was contracted, it was unfortunately not recorded in the authorities' standard debt management database. This led to due repayment alerts not being automatically triggered in the debt office. 
2. The new arrears accumulated by Burkina on June 2019 loan repayment give rise to the non-observance of the continuous performance criteria on accumulation of external payment arrears by government. Given the relatively small amount of the external payment arrears - 0.004 percent of GDP at end-June 2019 - and the repayment of the full amount of arrears, staff believes that this issue does not alter the overall assessment conducted in the staff report, including the authorities' continued commitment to the program. The authorities have promptly taken steps to verify and resolve the arrears. They contacted the Spanish Embassy in Abidjan (Côte d'Ivoire) and authorized payment of the arrears immediately. Further, to prevent recurrence of this issue in the future, the authorities reviewed their loan conventions to ensure that no other loan is missing from the database. Furthermore, the authorities envisage to review their procedures for recording loans in the debt database, and to strengthen debt reconciliation with creditors. Based on the above, staff supports the authorities' request for a waiver for the nonobservance on the basis of the minor and temporary nature of the breach and corrective measures put in place

3. A supplementary letter of intent is attached to this supplement. 


\section{Supplementary Letter of Intent}

Ouagadougou, July 16, 2019

Mr. David Lipton

Acting Managing Director

International Monetary Fund

Washington DC, 20431

Dear Mr. Acting Managing Director,

In addition to our Letter of Intent dated June 27, 2019, we wish to provide additional information on minor external arrears that were unintentionally accumulated to one of our bilateral creditors. The situation relates to external payment arrears for a loan agreement dating from 2008 with the Government of Spain that had not been entered in our standard debt management database. This led to due repayment alerts not being automatically triggered in the debt office.

As soon as the Government became aware of the existence of the aforementioned arrears, we have taken prompt measures to address them. The Government has immediately contacted the Spanish Embassy in Abidjan and authorized payment. The Government also reviewed its loan conventions to ensure that no other loan is missing from the database. Furthermore, the Government envisages to review its procedures for recording loans in the debt database, and to strengthen debt reconciliation with creditors to prevent any recurrence.

Against this background, I can confirm that the government of Burkina Faso paid an amount of $€ 494,383$ on July 16, 2019 and that no further external payments arrears beyond the above stated amount were incurred since the inception of the ECF arrangement.

I hereby request a waiver for the non-observance of the continuous performance criterion on non-accumulation of external arrears. I also take this opportunity to reaffirm our policy commitments under the ECF arrangement.

Sincerely,

$$
\text { /s/ }
$$

For the Minister of Economy, Finance and Development

The Minister Delegate in charge of Budget

Edith Clémence Yaka 


\section{Statement by Mr. Mohamed-Lemine Raghani, Executive Director for Burkina Faso, and Mr. Facinet Sylla, Alternate Executive Director and and Mr. Mamadou Siradiou Bah, Advisor to the Executive Director}

July 17, 2019

\section{Introduction}

On behalf of our Burkinabe authorities, we would like to express our appreciation for the Fund's continued support to Burkina Faso. Our authorities highly value the constructive discussions held with staff in Ouagadougou last May during the second review under the 2018-2020 Extended Credit Facility (ECF) arrangement.

Burkina Faso is steadfastly implementing its economic and financial program in a very challenging environment, including a difficult security situation in the region and strikes in the public sector. These developments have taken a heavy toll on the population and public investment. In particular, the insecurity in the region has led to a four-fold increase in the number of refugees and displaced populations between December 2018 and May 2019.

Despite this difficult situation, the program performance was satisfactory. Against this backdrop and their renewed commitment to achieve the program's objectives, our Burkinabe authorities are requesting the completion of the second review.

\section{Recent Economic Developments}

The growth momentum of the Burkinabe economy has continued in 2018, supported mainly by the rebound in the non-cotton agriculture production. Real GDP growth reached 6.8 percent against 6.3 percent in 2017. Inflation remained low due, among other factors, to the good agricultural harvest and measures to contain the cost of living.

The overall fiscal deficit improved significantly in 2018 to 4.9 percent of GDP from 7.8 percent a year earlier. This resulted from an increase in revenue collection and a notable reduction in current transfers and public investment. Nonetheless, the wage bill grew by 9.0 percent, reflecting the implementation of various salary agreements concluded in previous years with some civil service unions.

On the external front, the current account deficit declined to 5.8 percent of GDP in 2018 from 7.3 percent of GDP in 2017, mainly on the back of higher gold and agricultural exports as well as increased official transfers in the form of budget support. These positive developments helped strengthen the regional central bank's foreign reserves.

The banking system remains broadly sound. In particular, the aggregate capital level in 2018 stood above prudential requirements while the ratio of non-performing loans was contained at its 2017 level of 8.7 percent of total loans. 


\section{Program Performance}

Program performance in the period under review has been satisfactory. All quantitative performance criteria (PCs) and indicative targets set for end-December 2018 have been met except the PC related to the ceiling on net domestic financing of the government. It is also worth noting that 8 out of 13 structural benchmarks through mid-June 2019 were observed.

To address the causes of the missed PC, the authorities have introduced a strengthened mechanism to better monitor the public investment programming. The planning and spreading of commitments over the year will also be enhanced. The authorities, with the Fund technical assistance, will assess the adequacy of expenditure monitoring and execution mechanisms to avoid the excessive accumulation of committed expenditures without payment orders $(D E N O)$. Our Burkinabe authorities request a waiver for non-observance of this performance criterion.

\section{Economic Outlook and Risks}

The economic outlook for the near and medium-term remains favorable. Real GDP growth is projected at 6 percent in 2019 and in the medium-term, supported by the recovery of cotton production and higher mining production. Inflation is projected to remain below the WAEMU convergence criterion of 3 percent. Continued consolidation efforts will help meet the regional fiscal deficit convergence criterion of 3 percent of GDP in 2019. The current account deficit is expected to improve over the medium-term owing to the impact of fiscal consolidation on import demand and steady increases in mining and agriculture exports.

Mindful of the downside risks, including security challenges, climate change and rising protectionism, our authorities will pursue their efforts to preserve macroeconomic stability and strengthen the economy's resilience.

\section{Policies and Reforms for 2019 and Beyond}

\section{Fiscal Policy}

The authorities will implement a prudent and growth-friendly fiscal policy. As stated in the 2019 budget law, measures will be taken to boost domestic revenue mobilization, including by broadening the tax base and enhancing tax administration. In this regard, key measures will encompass expanding taxes on mining activities and enhancing the yield of the property tax on buildings and unbuilt land. Reforms to strengthen the tax administration will focus on further developing e-services and improving tax payment compliance.

On the expenditure side, actions are being taken to streamline current spending through the reduction of the wage bill and the government utilities bills. Regarding the wage bill and the reform of the civil service, the authorities remain determined to fully implement the 2018 recommendations of the national conference on the remuneration system. They have already published the results of the census of civil servants according to grade, region and ministry. They have also undertaken a comprehensive communication campaign to inform all stakeholders on the objectives of the civil service reform. Moreover, the authorities have 
committed to contain the public wage bill in 2019 and 2020 within the levels agreed during the first program review.

The authorities have developed a 2018-2020 action plan to enhance public financial management based on the results of the PEFA exercise. They will also reinforce the legal and regulatory expenditure procedures while streamlining the procurement process and improving budget execution.

\section{Debt Management Policy}

The authorities' debt management policy aims at preserving the country's moderate risk of debt distress over the medium-term. In this regard, they will pursue a prudent borrowing strategy by giving priority to concessional resources. In addition, they will continue their efforts to improve debt management capacity with the assistance of partners.

\section{Financial Inclusion}

Financial inclusion remains among the government's top priorities. The authorities plan to organize a roundtable to mobilize resources for the implementation of the National Inclusive Finance Strategy adopted last April. In their efforts to further promote access to credit, they have established a Credit Information Bureau. In addition, the two newly created financial institutions, Banque Agricole du Faso (BADF) and Caisse des Dépôts et Consignations $(C D C)$ have become operational.

\section{Structural Reforms}

In 2018, the authorities adopted a price adjustment mechanism for the petroleum products to reduce the hydrocarbon subsidies and ensure the financial stability of SONABHY (Société Nationale Burkinabè d'hydrocarbures). They remain fully committed to improve the operationalization of this adjustment mechanism as it will help release additional resources to finance public investment and support social sectors. A sustainable scheme will be introduced to mitigate the impact of the adjustment mechanism on the vulnerable groups. Regarding the overall energy sector, the authorities will continue to implement the Memorandum of Understanding on financial relations and hydrocarbon sale prices signed by the government, the electricity company SONABEL (Société Nationale d'Electricité du Burkina Faso) and SONABHY.

As regards governance, the authorities are committed to step up their efforts to fight corruption. The High Authority for State Control and Anti-Corruption (ASCE-LC) has been strengthened and the reports of the annual management audit of the government are posted on its website. The government intends to organize by November 2019 a symposium to improve coordination between the judiciary system and control units within the ministries. Efforts to further enhance the AML/CFT framework will be pursued, including strengthening the capacities of the coordinator body, CENTIF (Cellule Nationale de Traitement des Informations Financières), and addressing the framework's weaknesses based on the recommendations of the CENTIF's 2018 report. 


\section{Poverty Reduction}

The authorities are keen to make further progress in poverty reduction through the promotion of decent employment and the expansion of social protection. They also intend to reduce fragility by implementing the Sahel Emergency Program (PUS-BF) and address disparities among regions through the Local Economies Development Support Program (PADEL).

In response to the local humanitarian crisis caused by the heightened insecurity in the Sahel, the government has been taking various actions to assist refugees and internally displaced populations with the support of development partners.

\section{Conclusion}

Our authorities are determined to continue implementing their ECF-supported program. In view of this commitment and the progress achieved thus far, they are seeking the support of Executive Directors for the completion of the second review under the ECF arrangement. 